\title{
Propriedade Dunford-Pettis Alternativa
}

\author{
Veronica Leão Neves \\ DisSERTAÇÃO APRESENTADA \\ $\mathrm{AO}$ \\ Instituto DE MATEMÁticA E EstatísticA \\ DA \\ Universidade DE SÃo PAUlo \\ PARA \\ OBTENÇÃO DO TÍTULO \\ DE \\ Mestre em MatemáticA \\ Área de Concentração: Análise \\ Orientadora: Prof ${ }^{a}$ Dr $^{\mathrm{a}}$ Mary Lilian Lourenço
}

Durante o desenvolvimento deste trabalho a autora recebeu auxílio financeiro da FAPESP

-São Paulo, 24 de agosto de 2015- 


\section{Propriedade Dunford-Pettis Alternativa}

Esta versão da dissertação contém as correções e alterações sugeridas pela Comissão Julgadora durante a defesa da versão original do trabalho, realizada em 26/06/2015. Uma cópia da versão original está disponível no

Instituto de Matemática e Estatística da Universidade de São Paulo.

Comissão Julgadora:

- Prof ${ }^{\mathrm{a}}$. Dr ${ }^{\mathrm{a}}$. Mary Lilian Lourenço (orientadora) - IME-USP

- Prof. Dr. Humberto Daniel Carrión Villarroel - IME-USP

- Prof. Dr. Vinícius Vieira Fávaro - UFU 


\section{Agradecimentos}

Primeiramente, agradeço a Deus por ter me dado tantas oportunidades de crescer, em especial, de estudar Matemática e por ter colocado tantas pessoas maravilhosas na minha vida que me ajudariam nesta jornada.

Agradeço à minha orientadora Mary Lilian pela disponibilidade, atenção, companherismo e confiança. Obrigada pelos livros, ideias, sugestões, tempo e paciência! Aprendi muito sob a sua orientação. Também agradeço ao Professor Antonio M. Peralta, autor de dois dos artigos que estudei e muitos outros artigos que consultei. Ele foi muito atencioso ao esclarecer dúvidas que surgiram.

Agradeço aos professores dedicados que tive durante toda a minha formação, inclusive a minha orientadora. Professores que preparavam as aulas, que se importavam com as dúvidas, que se tornavam disponíveis fora do horário de aula e que ministraram excelentes aulas. A esses o merecido título de educadores, pois compreendem a responsabilidade que têm para a formação de futuros pesquisadores. A esses bons professores agradeço por fazerem meus olhos brilharem. Espero refletir o vosso brilhantismo, competência profissional e humana.

Aos meus verdadeiros amigos que me apoiaram e me acompanharam nesta jornada. Rimos, estudamos e aprendemos juntos. Foram momentos únicos que estarão para sempre conosco, pois fazem parte do que somos hoje. Talvez a maior lição que aprendemos é que leva tempo para aprender a essência e incorporar alguns conceitos. Mas é imensurável a satisfação diária de ampliar o conhecimento e dividi-lo com outras pessoas.

Em especial, agradeço à minha família, que me ensinou a importância de lutar, perseverar e estudar. Eles sempre me apoiaram, acreditaram em mim e proporcionaram um ambiente propício ao estudo e aprendizado em nosso lar. Devo tudo o que sou aos meus pais, sem eles eu não estaria aqui.

Quero agradecer, sobretudo, ao meu noivo Rafael, que esteve sempre ao meu lado me ajudando em tudo e que nunca me deixou desistir. Nos muitos momentos em que as coisas não saíram como eu gostaria e eu não sabia o que fazer, ele esteve sempre pronto para fazer tudo ao alcance dele 
para me socorrer e me ajudar a achar o caminho, com muita paciência e amor. Também esteve ao meu lado nos momentos em que a única coisa que precisava era tirar a cara dos livros e arejar os pensamentos. Sem o apoio dele eu não teria chegado até aqui. Muito obrigada por tudo!

Agradeço à FAPESP pelo auxílio financeiro. 


\section{Resumo}

NEVES, V. L. Propriedade Dunford-Pettis Alternativa. 2015. Dissertação de Mestrado - Instituto de Matemática e Estatística, Universidade de São Paulo, São Paulo, 2015.

Este trabalho tem como objetivo estudar a propriedade Dunford-Pettis alternativa (propriedade DP1), como introduzida por Freedman [21], e algumas de suas caracterizações e relações com outras propriedades.

Estudamos caracterizações para alguns espaços de operadores com a propriedade DP1, dadas por Acosta e Peralta em [1]. Vimos que um subespaço fechado do espaço dos operadores compactos em um espaço de Banach reflexivo com base de Schauder tem a propriedade DP1 se, e somente se, os operadores avaliação são operadores DP1. Estudamos um resultado análogo para espaços de Hilbert.

Como consequência desses resultados, vimos uma caracterização de certas subálgebras fechadas da álgebra dos operadores compactos que possuem a propriedade DP1, supondo que os operadores composição à direita e à esquerda são operadores DP1.

Finalmente, estudamos a demonstração feita por Bunce e Peralta em [10] de que as propriedades Dunford-Pettis e Duford-Pettis alternativa são equivalentes em $C^{*}$-álgebras.

Palavras-chave: Propriedade Dunford-Pettis, propriedade Dunford-Pettis alternativa, operadores compactos, $C^{*}$-álgebras. 


\section{Abstract}

NEVES, V. L. The Alternative Dunford-Pettis Property. 2015. Dissertação de Mestrado Instituto de Matemática e Estatística, Universidade de São Paulo, São Paulo, 2015.

The main purpose of this work is to study the alternative Dunford-Pettis property (DP1 property), as introduced by Freedman in [21], and some characterizations of the DP1 property and relations of this to other properties.

We studied a characterization of certain operator subspaces which have the DP1 property, as given by Acosta and Peralta in [1]. We saw that a closed subspace of the compact operators space in a reflexive Banach space with Schauder basis has the DP1 property if, and only if, the evaluation operators are DP1 operators. We studied a similar result for Hilbert spaces.

Consequently, we also saw a characterization of certain closed subalgebras of the compact operators algebra, in which the DP1 property is held by assuming that the right and left composition operators are DP1.

Finally, we studied the proof given by Bunce and Peralta in [10] that the Dunford-Pettis property and the alternative Duford-Pettis property are equivalent for $C^{*}$-algebras.

Keywords: The Dunford-Pettis property, the alternative Dunford-Pettis property, compact operators, $C^{*}$-algebras. 


\section{Sumário}

$\begin{array}{ll}\text { Lista de Símbolos } & \text { ix }\end{array}$

Introdução $\quad$ xiii

1 Tópicos de Análise Funcional $\quad 1$

1.1 Resultados em Espaços de Banach . . . . . . . . . . . . . . . . . . . 1

1.2 Base de Schauder . . . . . . . . . . . . . . . . . . . . . . . 8

1.3 Resultados em Espaços de Hilbert . . . . . . . . . . . . . . . . . . . . . . 9

1.4 Álgebras de Banach . . . . . . . . . . . . . . . . . . . 11

2 Propriedade Dunford-Pettis Alternativa $\quad 15$

2.1 Propriedade Dunford-Pettis . . . . . . . . . . . . . . . . 15

2.2 Propriedade Dunford-Pettis Alternativa . . . . . . . . . . . . . . . . 17

3 Subespaços Fechados do Espaço dos Operadores Compactos com Propriedade

Dunford-Pettis Alternativa

3.1 Subespaços Fechados do Espaço dos Operadores Compactos em Espaços de Banach com Propriedade Dunford-Pettis Alternativa . . . . . . . . . . . . . . . . 35

3.2 Subespaços Fechados do Espaço dos Operadores Compactos em Espaços de Hilbert com Propriedade Dunford-Pettis Alternativa . . . . . . . . . . . . . . . 47

3.3 Subálgebras Fechadas da Álgebra dos Operadores Compactos com Propriedade DunfordPettis Alternativa . . . . . . . . . . . . . . . . . . . . . 59

4 Propriedade Dunford-Pettis Alternativa em $C^{*}$-Álgebras $\quad 65$

$4.1 C^{*}$-Álgebras . . . . . . . . . . . . . . . . . . . . . . 65

4.2 Teoria das Representações e o Teorema de Gelfand-Naimark-Segal . . . . . . . . . 71

4.3 Álgebras de von Neumann . . . . . . . . . . . . . . . . . . . . . . . . . . 74 
viii SUMÁRIO

4.4 Equivalência das Propriedades Dunford-Pettis e Dunford-Pettis Alternativa em $C^{*}$ Álgebras . . . . . . . . . . . . . . . . . . . . . . . . . 75

Referências Bibliográficas 


\title{
Lista de Símbolos
}

\author{
$\mathbb{N} \quad$ o conjunto dos números inteiros estritamente positivos \\ $\mathbb{R} \quad$ o corpo dos números reais \\ $\mathbb{C} \quad$ o corpo dos números complexos \\ $\mathbb{K} \quad$ o corpo $\mathbb{R}$ ou $\mathbb{C}$ \\ $X, Y \quad$ espaços normados ou de Banach sobre $\mathbb{K}$ \\ $B_{X} \quad$ a bola unitária fechada de $X$ \\ $S_{X} \quad$ a esfera unitária de $X$ \\ $\bar{W} \quad$ fecho do subconjunto $W$ de $X$

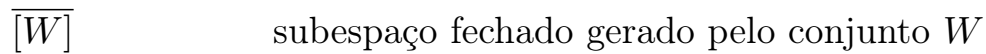 \\ $M^{\perp} \quad$ o complemento ortogonal do subconjunto $M$ de $X$ \\ $L(X, Y) \quad$ o espaço de Banach de todos os operadores lineares e contínuos de $X$ em $Y$ \\ com a norma $\|T\|=\sup _{x \in B_{X}}\|T(x)\|$, para todo $T \in L(X, Y)$ \\ $L(X) \quad$ o espaço de Banach de todos os operadores lineares e contínuos de $X$ em $X$ \\ $K(X, Y) \quad$ o subespaço fechado de todos os operadores lineares compactos de $X$ em $Y$ \\ $K(X) \quad$ o subespaço fechado de todos os operadores lineares compactos de $X$ em $X$ \\ $K_{w}(X, Y) \quad$ o subespaço fechado de todos os operadores lineares $w$-compactos de $X$ em $Y$ \\ $X^{*} \quad$ o dual topológico de $X$ \\ $X^{* *} \quad$ o bidual topológico de $X$ \\ $A^{\prime} \quad$ o comutante de uma álgebra $A$ \\ $A^{\prime \prime} \quad$ o bicomutante de uma álgebra $A$ \\ $C: X \rightarrow X^{* *} \quad$ a aplicação canônica de $X$ em $X^{* *}$ tal que $C(x)=g_{x}$, \\ em que $g_{x}(\varphi)=\varphi(x)$, para todo $\varphi \in X^{*}$ \\ $x_{n} \longrightarrow x \quad$ a sequência $\left(x_{n}\right)_{n}$ converge para $x$ em $X$ \\ $x_{n} \nrightarrow x \quad$ a sequência $\left(x_{n}\right)_{n}$ não converge para $x$ em $X$ \\ $x_{n} \stackrel{w}{\longrightarrow} x \quad$ a sequência $\left(x_{n}\right)_{n}$ converge fracamente para $x$ em $X$ \\ $\varphi_{n} \stackrel{w^{*}}{\longrightarrow} \varphi \quad$ a sequência $\left(\varphi_{n}\right)_{n}$ converge fraco-estrela para $\varphi$ em $X^{*}$
}




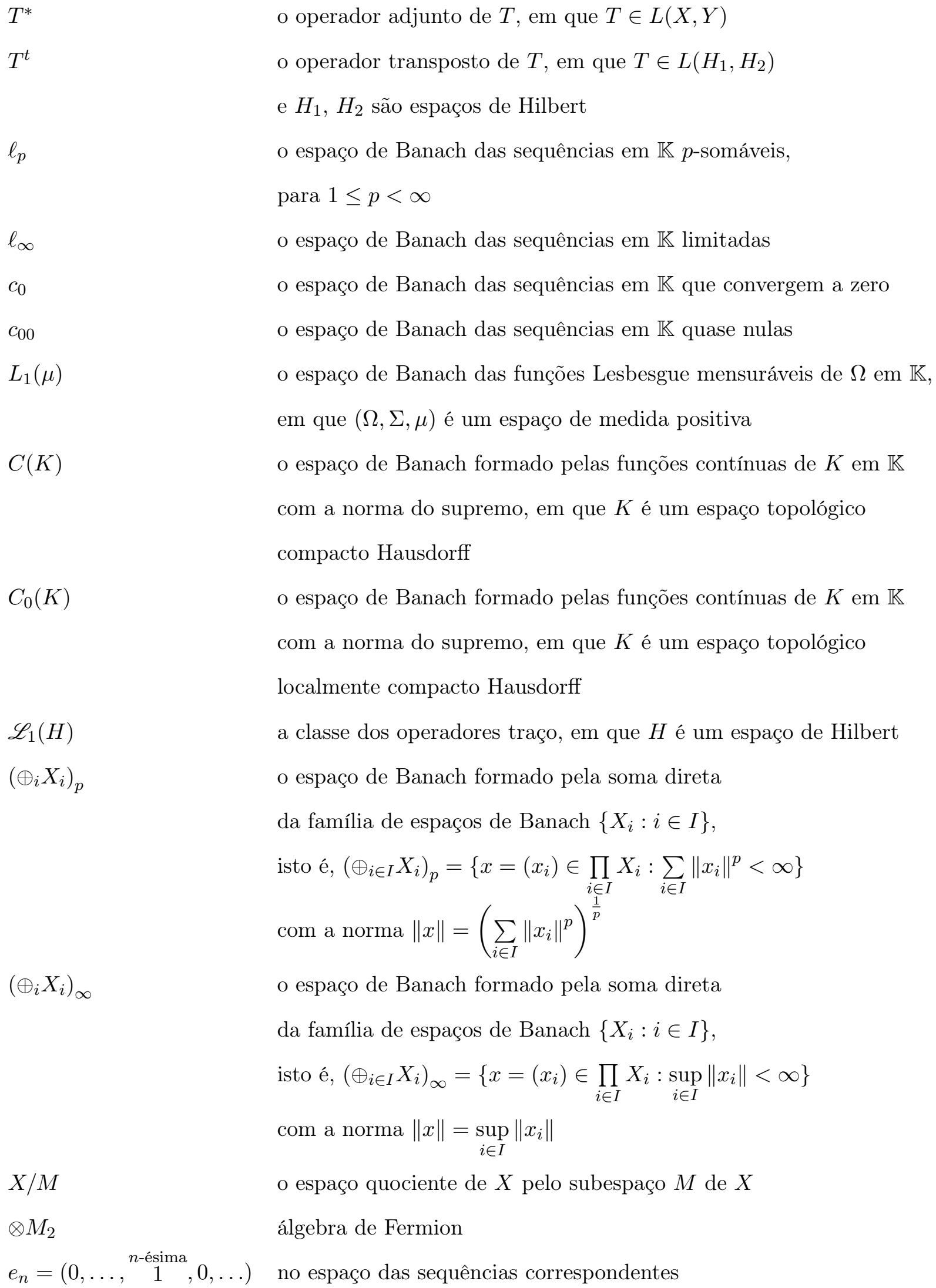




\section{Introdução}

Os matemáticos Nelson James Dunford e Billy James Pettis provaram, em 1940, no artigo [17] que as aplicações lineares $w$-compactas de $L_{1}(\mu)$ em $X$ são $w$-sequencialmente contínuas, sendo $\mu$ uma medida e $X$ um espaço de Banach.

Em 1953, Alexander Grothendieck provou, em [22], que todo operador w-compacto em $C(K)$, em que $K$ é um compacto Hausdorff, é $w$-sequencialmente contínuo. Assim, Grothendieck introduziu a definição de um espaço de Banach ter a propriedade de Dunford-Pettis (propriedade DP). Um espaço de Banach $X$ tem a propriedade DP se, para todo espaço de Banach $Y$ e toda aplicação linear $T: X \longrightarrow Y w$-compacta, $T$ aplica sequências $w$-Cauchy de $X$ em sequências de Cauchy em $Y$, isto é, $T$ é $w$-sequencialmente contínua. A partir dessa definição, muito foi estudado a respeito da propriedade DP.

Em 1997, Walden Freedman em [21] introduziu a propriedade Dunford-Pettis alternativa (propriedade DP1). Um espaço de Banach $X$ tem a propriedade DP1 se, dados $\left(x_{n}\right)_{n} \subset X$ e $x \in X$ tais que $\left\|x_{n}\right\|=\|x\|=1$, para todo $n \in \mathbb{N}$, e $x_{n} \stackrel{w}{\longrightarrow} x$ em $X$, e dada uma sequência $\left(f_{n}\right)_{n} \subset X^{*}$ e

dado $f \in X^{*}$, com $f_{n} \stackrel{w}{\longrightarrow} f$ em $X^{*}$, temos que $f_{n}\left(x_{n}\right) \longrightarrow f(x)$. Um dos objetivos de Freedman ao definir a propriedade DP1 era que vários pré-duais de álgebras de von Neumann não comutativas possuíssem tal propriedade. A propriedade DP1 é estritamente mais fraca do que a propriedade DP para espaços de Banach em geral, como será visto na Seção 2.2 por meio de exemplos. Entretanto, na Seção 4.4, veremos que elas são equivalentes em $C^{*}$-álgebras.

Freedman caracterizou também a propriedade DP1 por meio de operadores. Um operador $T \in$ $L(X, Y)$ é um operador DP1 se, dados $\left(x_{n}\right)_{n} \subset X$ e $x \in X$ tais que $\left\|x_{n}\right\|=\|x\|=1$, para todo $n \in \mathbb{N}$, e $x_{n} \stackrel{w}{\longrightarrow} x$ em $X$, temos que $T\left(x_{n}\right) \longrightarrow T(x)$. Um espaço de Banach $X$ tem a propriedade DP1 se, para todo espaço de Banach $Y$ e todo operador $T \in L(X, Y)$ w-compacto, temos que $T$ é um operador DP1. Assim, observamos que os operadores DP1 têm um papel importante no estudo da propriedade DP1, papel este análogo aos operadores $w$-sequencialmente contínuos com relação à propriedade DP. 
O objetivo desta dissertação é estudar a propriedade Dunford-Pettis alternativa, exemplos de espaços que possuem ou não a propriedade DP1, alguns resultados equivalentes à definição da propriedade DP1 e relações com as propriedades Dunford-Pettis e Kadec-Klee.

Descreveremos a seguir os assuntos abordados em cada capítulo. Convém salientar que os principais resultados aqui desenvolvidos encontram-se nos artigos [1], [10] e [21].

Será de fundamental importância desenvolver um capítulo com definições e resultados centrais de Análise Funcional que serão utilizadas para alcançarmos nossos objetivos. Esse será o conteúdo do Capítulo 1. Nas Seções 1.1, 1.2, 1.3 e 1.4 serão apresentados, respectivamente, os resultados e definições sobre espaços de Banach, base de Schauder, espaços de Hilbert e álgebras de Banach. Optamos por não apresentar as demonstrações de tais resultados, mas deixar referências aos leitores interessados.

Nosso objetivo no Capítulo 2 será introduzir a propriedade DP1 e apresentar alguns resultados iniciais. Na Seção 2.1, introduziremos a propriedade DP e alguns exemplos de espaços com a DP que serão importantes para relacionarmos as duas propriedades. Esse capítulo foi baseado principalmente em [24]. Na Seção 2.2, definiremos a propriedade DP1 e estudaremos alguns resultados equivalentes à definição da propriedade DP1. Veremos que a propriedade DP implica a DP1, mas que a recíproca não é verdadeira em geral. A propriedade DP1 é preservada por subespaços complementados, isomorfismo isométrico e espaço quociente (sob algumas condições sobre o espaço). Veremos que se $1 \leq p<\infty$, então a soma direta com a norma $p$ de espaços com a propriedade DP1 tem a propriedade DP1.

No Capítulo 3, estudaremos subespaços fechados do espaço dos operadores compactos com a propriedade DP1. Na Seção 3.1, subespaços fechados do espaço dos operadores compactos em espaços de Banach com a propriedade DP1 serão estudados. O principal resultado será que se $X$ e $Y$ são espaços de Banach reflexivos com bases de Schauder e $M$ é um subespaço fechado de $K(X, Y)$ satisfazendo a uma determinada propriedade, então $M$ possui a propriedade DP1 se, e somente se, os operadores avaliação são operadores DP1.

Analogamente, na Seção 3.2, estudaremos um resultado análogo para espaços de Hilbert, isto é, se $H$ um espaço de Hilbert (não necessariamente separável) e $M$ é um subespaço fechado de $K(H)$, então $M$ tem a propriedade DP1 se, e somente se, os operadores avaliação são operadores DP1.

Para uma subálgebra de Banach fechada $A$ de $K(X)$ ou $K(H)$, com algumas condições sobre $A$, veremos que $A$ tem a propriedade $\mathrm{DP} 1$ se, e somente se, os operadores composição à direita e à esquerda são operadores DP1. Essa caracterização da propriedade DP1 para subálgebras de Banach será apresentada na Seção 3.3. 
Por último, no Capítulo 4, estudaremos que as propriedades DP e DP1 são equivalentes em $C^{*}$ álgebras. Para tanto serão utilizados alguns resultados envolvendo $C^{*}$-álgebras e álgebras de von Neumann. Na Seção 4.1, estudaremos definições, resultados e exemplos envolvendo $C^{*}$-álgebras. Na Seção 4.2, veremos, brevemente, dois resultados centrais na teoria de $C^{*}$-álgebras que são os teoremas de Gelfand-Naimark-Segal e Gelfand-Naimark para $C^{*}$-álgebras comutativas, que mostram que toda $C^{*}$-álgebra pode ser vista como uma $C^{*}$-subálgebra de $L(H)$ e que toda $C^{*}$-álgebra comutativa é isometricamente isomorfa a $C_{0}(K)$. Na Seção 4.3, discorreremos sucintamente sobre as álgebras de von Neumann, enunciando alguns resultados importantes para o nosso trabalho. Entretando, omitiremos as demonstrações devido ao teor técnico que foge ao escopo deste trabalho. O principal resultado desse capítulo se encontra na Seção 4.4, na qual estudaremos a equivalência das propriedades DP e DP1 em $C^{*}$-álgebras. Encerraremos com uma caracterização das propriedades DP e DP1 em $C^{*}$-subálgebras da álgebra $K(H)$. 


\section{Capítulo 1}

\section{Tópicos de Análise Funcional}

Para tratar da propriedade que intitula esta dissertação serão necessários alguns resultados clássicos de Análise Funcional. O objetivo deste capítulo é fixar notações, definir conceitos e enunciar resultados que serão utilizados. Optamos por enunciar os resultados e deixar referências para as demonstrações.

Serão apresentados resultados e definições sobre espaços de Banach, base de Schauder, espaços de Hilbert e álgebras de Banach.

\subsection{Resultados em Espaços de Banach}

Nesta seção, serão apresentados resultados em espaços de Banach relevantes para este trabalho. Alguns resultados são mais gerais, mas nosso foco está nas consequências desses resultados para espaços de Banach. As principais referências são [7] e [26].

Iniciamos pelos teoremas centrais de Análise Funcional. Eles serão utilizados em várias demonstrações nos capítulos subsequentes.

Teorema 1.1.1 (Teorema de Banach-Steinhaus). Sejam $X$ um espaço de Banach, $Y$ um espaço normado e $\left(T_{i}\right)_{i \in I}$ uma família de operadores em $L(X, Y)$ satisfazendo à condição de que para cada $x \in X$ existe $C_{x}<\infty$ tal que $\sup _{i \in I}\left\|T_{i}(x)\right\|<C_{x}$, então $\sup _{i \in I}\left\|T_{i}\right\|<\infty$.

(vide [7, p. 38-39])

Teorema 1.1.2 (Teorema da Aplicação Aberta). Sejam $X, Y$ espaços de Banach e $T \in$ $L(X, Y)$ sobrejetora. Então $T$ é uma aplicação aberta. Além disso, se $T$ for injetora, segue que $T^{-1}$ é contínua, isto é, $T$ é um isomorfismo.

(vide [7, p. 42-43])

Teorema 1.1.3 (Teorema de Hahn-Banach). Sejam $X$ um espaço normado sobre $\mathbb{K}, Y$ um subespaço próprio de $X$ e $\phi \in Y^{*}$. Então existe $\varphi \in X^{*}$ tal que $\phi(y)=\varphi(y)$ para qualquer $y \in Y e$ $\|\phi\|=\|\varphi\|$.

(vide [7, p. 60])

Corolário 1.1.4. Sejam $X$ um espaço normado sobre $\mathbb{K}$ e $x_{0} \in X$ tal que $x_{0} \neq 0$. Então existe um funcional linear $\varphi \in X^{*}$ tal que $\|\varphi\|=1$ e $\varphi\left(x_{0}\right)=\left\|x_{0}\right\|$.

(vide [7, p. 60]) 
A seguir, abordaremos os conceitos de projeção, subespaço complementado, cópia isomorfa, operador adjunto e espaço reflexivo.

Definição 1.1.5. Seja $X$ um espaço de Banach. Um operador linear contínuo $P: X \longrightarrow X$ é uma projeção se $P^{2}=P \circ P=P$.

Observe que se $P \neq 0$ é uma projeção, então $\|P\| \geq 1$.

Proposição 1.1.6. Sejam $X$ um espaço de Banach e $Y$ um subespaço de $X$. As seguintes afirmações são equivalentes:

(a) Existe uma projeção $P: X \longrightarrow X$ cuja imagem coincide com $Y$. Neste caso, dizemos que $P$ é uma projeção de $X$ sobre $Y$;

(b) $Y$ é fechado e existe um subespaço fechado $Z$ de $X$ tal que $X=Y \oplus Z$. Neste caso, $Y=\{x \in$ $X: P(x)=x\}$ e $Z=N u c(P)$.

(vide [\%, p. 61])

Definição 1.1.7. Sejam $X$ um espaço de Banach e $Y$ um subespaço de $X$. Dizemos que $Y$ é complementado em $X$ se satisfaz às condições equivalentes da Proposição 1.1.6.

Vejamos exemplos de subespaços complementados e não complementados. Para tanto definiremos um tipo especial de operador linear que será usado num dos exemplos e em outros momentos ao longo do texto.

Definição 1.1.8. Sejam $X, Y$ espaços de Banach, $\varphi \in X^{*}$ e $y \in Y$. Definimos o operador linear $\varphi \otimes y: X \longrightarrow Y$ dado por $\varphi \otimes y(x)=\varphi(x) y$.

Afirmamos que $\varphi \otimes y$ é contínuo, de posto 1, e de norma $\|\varphi\|\|y\|$. De fato,

$$
\|\varphi \otimes y\|=\sup _{\|x\|=1}\|\varphi \otimes y(x)\|=\sup _{\|x\|=1}\|\varphi(x) y\|=\sup _{\|x\|=1}|\varphi(x)|\|y\|=\|\varphi\|\|y\| .
$$

Exemplo 1.1.9. (a) Todo subespaço de dimensão finita de um espaço de Banach é complementado. Seja $Y$ um subespaço de dimensão $n$ do espaço de Banach $X$ e $\left\{e_{1}, \ldots, e_{n}\right\}$ uma base para $Y$. Para cada $j=1, \ldots, n$, considere o funcional linear contínuo $\varphi_{j} \in Y^{*}$ dado por $\varphi_{j}\left(\sum_{k=1}^{n} a_{k} e_{k}\right)=a_{j}$. Pelo Teorema de Hahn-Banach (Teorema 1.1.3), existe $\tilde{\varphi}_{j} \in X^{*}$ extensão de $\varphi_{j}, j=1, \ldots, n$. O operador $P:=\sum_{j=1}^{n} \tilde{\varphi}_{j} \otimes e_{j}$ é uma projeção de $X$ sobre $Y$.

(b) Segue da Proposição 1.1.6 (b) que todo subespaço complementado de um espaço de Banach é fechado. Assim, um subespaço não-fechado de um espaço de Banach não é complementado. Por exemplo, $c_{00}$ não é complementado em $c_{0}$.

Definição 1.1.10. Sejam $X$ e $Y$ espaços de Banach. Dizemos que $X$ tem uma cópia (isomorfa) de $Y$, o que denotamos por $Y \hookrightarrow X$, se existe $T \in L(Y, X)$ injetora tal que $T^{-1}: T(Y) \longrightarrow Y$ é contínua. 
Definição 1.1.11. Sejam $X, Y$ espaços vetoriais normados e $T \in L(X, Y)$ um operador linear contínuo. Definimos o operador $T^{*}: Y^{*} \longrightarrow X^{*}$ por

$$
T^{*}(\varphi)(x)=\varphi(T(x)) \text { para todo } x \in X \text { e } \varphi \in Y^{*} .
$$

O operador $T^{*}$ é chamado adjunto de $T$.

Proposição 1.1.12. Sejam $X, Y$ e $Z$ espaços vetoriais normados, $T, S \in L(X, Y), R \in L(Y, Z)$ e $\alpha \in \mathbb{K}$. Então:

(a) $(T+S)^{*}=T^{*}+S^{*}$;

(b) $(\alpha T)^{*}=\alpha T^{*}$;

(c) $(R S)^{*}=S^{*} R^{*}$.

(vide [26, p. 285])

Definição 1.1.13. Seja $X$ um espaço de normado. Definimos $C: X \rightarrow X^{* *}$ a aplicação canônica de $X$ em $X^{* *}$ tal que $C(x)=g_{x}$, em que $g_{x}(\varphi)=\varphi(x)$, para todo $\varphi \in X^{*}$.

Proposição 1.1.14. A aplicação canônica $C: X \rightarrow X^{* *}$ é linear, injetora e uma isometria, isto é, $\|C(x)\|=\left\|g_{x}\right\|=\|x\|$, para todo $x \in X$.

(vide [7, p. 89])

De modo geral, $C$ não é sobrejetora. Assim, se $C$ for sobrejetora, dizemos que o espaço $X$ é reflexivo. Todo espaço reflexivo é um espaço de Banach.

No capítulo 3, estudaremos a propriedade DP1 para subespaços do espaço dos operadores compactos. Assim, definiremos operadores compactos e enunciaremos alguns resultados relacionados.

Definição 1.1.15. Sejam $X$ e $Y$ espaços de Banach. Um operador linear $T: X \rightarrow Y$ é denominado operador compacto se, para todo subconjunto limitado $W$ de $X$, temos que $T(W)$ é relativamente compacto em $Y$, ou seja, $\overline{T(W)}$ é compacto em $Y$.

O espaço de todos os operadores lineares compactos de $X$ em $Y$ será denotado por $K(X, Y)$.

Teorema 1.1.16 (Schauder). Sejam $X, Y$ espaços normados e $T \in L(X, Y)$. Então, $T$ é compacto se, e somente se, $T^{*}$ é compacto.

(vide [7, p. 190])

Proposição 1.1.17. Sejam $X, Y$ espaços de Banach e $T$ um operador linear de $X$ em $Y$. Então:

(a) Se $T$ é contínuo e $\operatorname{dim} T(X)<\infty$, temos que $T$ é compacto;

(b) Se $\operatorname{dim} X<\infty$, temos que $T$ é compacto.

(vide [14, p. 178])

Proposição 1.1.18. Sejam $X$ e $Y$ espaço normados. Se $Y$ é um espaço de Banach, então $K(X, Y)$ é um subespaço fechado de $L(X, Y)$.

(vide [\%, p. 189]) 
Proposição 1.1.19. Sejam $X, Y$ espaços de Banach, $\left(T_{n}\right)_{n} \subseteq L(X, Y)$ e $T \in L(X, Y)$. Se $T_{n}(x) \longrightarrow$ $T(x)$, para todo $x \in X$, então, para todo compacto $K \subset X, T_{n}$ converge uniformemente a $T$ em $K$, isto é, $\sup _{x \in K}\left\|T_{n}(x)-T(x)\right\| \longrightarrow 0$.

(vide [19, p. 204])

No capítulo 2, será usado o conceito de soma direta de espaços de Banach. Assim, no que segue, apresentamos alguns destes conceitos.

Definição 1.1.20. Sejam $X_{1}, \ldots, X_{n}$ espaços normados com normas $\|\cdot\|_{X_{1}}, \ldots,\|\cdot\|_{X_{n}}$, respectivamente. A soma direta de $X_{1}, \ldots, X_{n}$ é o produto cartesiano $X_{1} \times \cdots \times X_{n}$ com norma

$$
\left\|\left(x_{1}, \ldots, x_{n}\right)\right\|=\left(\sum_{j=1}^{n}\left\|x_{j}\right\|_{X_{j}}^{2}\right)^{\frac{1}{2}} .
$$

Esse espaço normado é denotado por $X_{1} \oplus \cdots \oplus X_{n}$.

Os subescritos das normas de $X_{1}, \ldots, X_{n}$ serão omitidos.

Proposição 1.1.21. Sejam $X_{1}, \ldots, X_{n}$ espaços normados $e 1 \leq p \leq \infty$. Então

$$
\begin{aligned}
\left\|\left(x_{1}, \ldots, x_{n}\right)\right\|_{p} & =\left(\sum_{j=1}^{n}\left\|x_{j}\right\|^{p}\right)^{\frac{1}{p}} \\
\left\|\left(x_{1}, \ldots, x_{n}\right)\right\|_{\infty} & =\max \left\{\left\|x_{1}\right\|, \ldots,\left\|x_{n}\right\|\right\}
\end{aligned}
$$

são normas para a soma de $X_{1}, \ldots, X_{n}$, equivalentes à norma usual da soma direta definida acima. (vide [26, p. 68])

Teorema 1.1.22. Sejam $X_{1}, \ldots, X_{n}$ espaços normados. Então $X_{1} \oplus \cdots \oplus X_{n}$ é um espaço de Banach se, e somente se, cada $X_{j}$ é um espaço de Banach.

(vide [26, p. 63])

Teorema 1.1.23. Sejam $X_{1}, \ldots, X_{n}$ espaços normados. Então $X_{1} \oplus \cdots \oplus X_{n}$ é reflexivo se, $e$ somente se, cada $X_{j}$ é reflexivo.

(vide [26, p. 105])

Definição 1.1.24. Sejam $X_{1}, \ldots, X_{n}, Y_{1}, \ldots, Y_{n}$ espaços normados e $T_{j}$ operadores lineares de $X_{j}$ em $Y_{j}, j=1, \ldots, n$. Ent $\tilde{a} o$

$$
T_{1} \oplus \cdots \oplus T_{n}: X_{1} \oplus \cdots \oplus X_{n} \longrightarrow Y_{1} \oplus \cdots \oplus Y_{n}
$$

dado por $T_{1} \oplus \cdots \oplus T_{n}\left(x_{1}, \ldots, x_{n}\right)=\left(T_{1}\left(x_{1}\right), \ldots, T_{n}\left(x_{n}\right)\right)$, em que $\left(x_{1}, \ldots, x_{n}\right) \in X_{1} \oplus \cdots \oplus X_{n}$. Temos que $T_{1} \oplus \cdots \oplus T_{n}$ é linear.

Teorema 1.1.25. Sejam $X_{1}, \ldots, X_{n}, Y_{1}, \ldots, Y_{n}$ espaços normados e $T_{j}$ operadores lineares de $X_{j}$ em $Y_{j}, j=1, \ldots, n$. Então $T_{1} \oplus \cdots \oplus T_{n}$ é contínuo se, e somente se, cada $T_{j}$ é contínuo. Além disso, se $T_{1} \oplus \cdots \oplus T_{n}$ é contínuo, então $\left\|T_{1} \oplus \cdots \oplus T_{n}\right\|=\max \left\{\left\|T_{1}\right\|, \cdots,\left\|T_{n}\right\|\right\}$.

(vide [26, p. 66-6r]) 
Teorema 1.1.26. Sejam $X_{1}, \ldots, X_{n}$ espaços normados. Então, existe um isomorfismo isométrico que identifica $\left(X_{1} \oplus \cdots \oplus X_{n}\right)^{*}$ com $X_{1}^{*} \oplus \cdots \oplus X_{n}^{*}$ tal que se $\varphi \in\left(X_{1} \oplus \cdots \oplus X_{n}\right)^{*}$ está identificado com $\left(\varphi_{1}, \ldots, \varphi_{n}\right) \in X_{1}^{*} \oplus \cdots \oplus X_{n}^{*}$, então $\varphi\left(x_{1}, \ldots, x_{n}\right)=\sum_{j=1}^{n} \varphi_{j}\left(x_{j}\right)$, em que $\left(x_{1}, \ldots, x_{n}\right) \in X_{1} \oplus \cdots \oplus X_{n}$. (vide [26, p. 91])

O conceito de rede será importante para a caracterização de compacidade e também será utilizado em outros momentos ao longo do trabalho.

Definição 1.1.27. Dizemos que um conjunto parcialmente ordenado $(\Lambda, \leq)$ é dirigido, se dados $\beta, \gamma \in \Lambda$, existe $\lambda \in \Lambda$ tal que $\beta \leq \lambda$ e $\gamma \leq \lambda$.

Uma rede em um espaço topológico $\mathcal{X}$ é uma função $\lambda \in \Lambda \longmapsto x_{\lambda} \in \mathcal{X}$, em que $\Lambda$ é um conjunto dirigido.

De forma análoga à notação de sequências, denotamos uma rede por $\left(x_{\lambda}\right)_{\lambda \in \Lambda}$.

Dizemos que uma rede $\left(x_{\lambda}\right)_{\lambda \in \Lambda} \subset \mathcal{X}$ converge para $x \in \mathcal{X}$, se para toda vizinhança aberta $V$ de $x$ em $\mathcal{X}$, existe $\lambda_{0} \in \Lambda$ tal que, se $\lambda \geq \lambda_{0}$ então $x_{\lambda} \in V$. Denotamos por $x_{\lambda} \longrightarrow x$ ou $\lim _{\lambda} x_{\lambda}=x$.

Definição 1.1.28. Seja $\left(x_{\lambda}\right)_{\lambda \in \Lambda}$ uma rede em um espaço topológico $\mathcal{X}$. Dizemos que $x \in \mathcal{X}$ é um ponto de acumulação de $\left(x_{\lambda}\right)$ se para toda vizinhança $V$ de $x$ em $\mathcal{X}$ e todo $\lambda \in \Lambda$, existe $\lambda_{0} \in \Lambda$ tal que $\lambda \leq \lambda_{0}$ e $x_{\lambda_{0}} \in V$.

Teorema 1.1.29. Um espaço topológico Hausdorff $\mathcal{X}$ é compacto se, e somente se, toda rede em $\mathcal{X}$ tem um ponto de acumulação.

(vide [18, p. 128])

Serão apresentadas as topologias fraca e fraca-estrela e alguns resultados importantes para este trabalho.

Definição 1.1.30. Seja $X$ um espaço vetorial normado. A topologia fraca de $X$, denotada por $\sigma\left(X, X^{*}\right)$, é a topologia obtida tomando como base todos os conjuntos da forma:

$$
V\left(x_{0} ; f_{1}, f_{2}, \ldots, f_{n} ; \epsilon\right)=\left\{x \in X: \sup _{1 \leq i \leq k}\left|f_{i}(x)-f_{i}\left(x_{0}\right)\right|<\epsilon\right\},
$$

sendo $x_{0} \in X, f_{1}, f_{2}, \ldots, f_{n} \in X^{*} e \epsilon>0$.

O conjunto aberto, fechado, compacto, função contínua, operador compacto, rede convergente, sequência de Cauchy com relação à topologia fraca serão denotados por $w$-aberto, $w$-fechado, $w$-compacto, função $w$-contínua, operador $w$-compacto, rede $w$-convergente, sequência $w$-Cauchy. Além disso, denotaremos por $\bar{A}^{w}$, o fecho fraco do conjunto $A$.

O espaço topológico $(X, w)$ é um espaço Hausdorff e o operador $I d:(X,\|\cdot\|) \longrightarrow(X, w)$ é contínuo ou, equivalentemente, todo aberto em $(X, w)$ contém um aberto de $(X,\|\cdot\|)$.

Proposição 1.1.31. Uma rede $\left(x_{\lambda}\right)_{\lambda \in \Lambda}$ em um espaço vetorial normado $X$ é $w$-convergente para $x \in X$, denotamos por $x_{\lambda} \stackrel{w}{\longrightarrow} x$, se, e somente se, para todo $f \in X^{*}$, temos que $f\left(x_{\lambda}\right) \longrightarrow f(x)$.

(vide [7, p. 144]) 
Definição 1.1.32. Seja $X$ um espaço vetorial normado. A topologia fraca-estrela de $X^{*}$, denotada por $\sigma\left(X^{*}, X\right)$, é a topologia obtida tomando como base todos os conjuntos da forma:

$$
W\left(f_{0} ; x_{1}, x_{2}, \ldots, x_{n} ; \epsilon\right)=\left\{f \in X^{*}: \sup _{1 \leq i \leq k}\left|f\left(x_{i}\right)-f_{0}\left(x_{i}\right)\right|<\epsilon\right\},
$$

sendo $f_{0} \in X^{*}, x_{1}, x_{2}, \ldots, x_{n} \in X e \epsilon>0$.

Uma rede $w^{*}$-convergente está se referindo a um rede convergente com relação à topologia fraca-estrela.

O espaço topológico $\left(X^{*}, w^{*}\right)$ é um espaço Hausdorff e o operador $I d:\left(X^{*}, w\right) \longrightarrow\left(X^{*}, w^{*}\right)$ é contínuo ou, equivalentemente, todo aberto em $\left(X^{*}, w^{*}\right)$ contém um aberto de $\left(X^{*}, w\right)$.

Proposição 1.1.33. Seja $X$ um espaço vetorial normado. Uma rede $\left(f_{\lambda}\right)_{\lambda \in \Lambda}$ em um $X^{*}$ é $w^{*}$ convergente para $f \in X^{*}$, denotamos por $f_{\lambda} \stackrel{w^{*}}{\longrightarrow} f$, se, e somente se, para todo $x \in X$, temos que $f_{\lambda}(x) \longrightarrow f(x)$.

(vide [7, p. 152])

Vejamos alguns resultados relacionados a sequências e às topologias fraca e fraca-estrela que serão utilizados posteriormente.

Proposição 1.1.34. Seja $X$ um espaço normado. Se $x_{n} \stackrel{w}{\longrightarrow} x$ em $X$, então a sequência $\left(x_{n}\right)_{n}$ é limitada em $X$ e $\|x\| \leq \liminf _{n}\left\|x_{n}\right\|$.

(vide [\%, p. 145])

Proposição 1.1.35. Seja $X$ um espaço normado.

(a) Em $X^{*}$, a topologia fraca-estrela $\sigma\left(X^{*}, X\right)$ está contida na topologia fraca $\sigma\left(X^{*}, X^{* *}\right)$;

(b) As topologias fraca $\sigma\left(X^{*}, X^{* *}\right)$ e fraca-estrela $\sigma\left(X^{*}, X\right)$ coincidem em $X^{*}$ se, e somente se, $X$ é reflexivo.

(vide [7, p. 155])

Decorre da proposição anterior que se $f_{n} \stackrel{w}{\longrightarrow} f$ em $X^{*}$, então $f_{n} \stackrel{w^{*}}{\longrightarrow} f$ em $X^{*}$. Usaremos esse fato várias vezes ao longo do texto.

Teorema 1.1.36 (Eberlein-Smulian). Sejam $X$ um espaço de Banach e $W$ um subconjunto de $X$. Então, $W$ é w-relativamente compacto se, e somente se, toda sequência $\left(x_{n}\right)_{n} \subseteq W$ tem uma subsequência w-convergente em $X$.

(vide [26, p. 248])

Corolário 1.1.37. Seja X um espaço de Banach. Então, $X$ é reflexivo se, e somente se, toda sequência limitada em $X$ tem uma subsequência $w$-convergente.

(vide [26, p. 251])

Definição 1.1.38. Seja $X$ um espaço normado.

(a) Dizemos que uma senquência $\left(x_{n}\right)_{n}$ em X é $\boldsymbol{w}$-Cauchy se $f\left(x_{n}\right)$ é um sequência de Cauchy em $\mathbb{K}$, para todo $f \in X^{*}$; 
(b) Dizemos que X éw-sequencialmente completo se toda sequência w-Cauchy éw-convergente em $X$.

Exemplo 1.1.39. Todo espaço de Banach reflexivo é $w$-sequencialmente completo. (vide [26, p. 251])

Teorema 1.1.40. Sejam $X$ um espaço de Banach e $M$ um subespaço fechado de $X$ tal que $M$ não contém cópia do espaço $\ell_{1}$. Então toda sequência $w$-Cauchy em $X / M$ tem uma subsequência que é a imagem de uma sequência $w$-Cauchy em $X$ pela projeção $\pi: X \longrightarrow X / M$.

(vide [16])

Teorema 1.1.41 (Banach-Alaoglu-Bourbaki). Para todo espaço normado $X$ a bola $B_{X^{*}} e$ compacta na topologia fraca-estrela $\sigma\left(X^{*}, X\right)$ de $X^{*}$.

(vide [7, p. 156])

Teorema 1.1.42 (Goldstine). Seja $X$ um espaço normado e $C$ a aplicação canônica de $X$ em $X^{* *}$. Então $C\left(B_{X}\right)$ é $w^{*}$-denso em $B_{X^{* *} \text {. }}$

(vide [26, p. 232])

Corolário 1.1.43. Seja $X$ um espaço normado e $C$ a aplicação canônica de $X$ em $X^{* *}$. Então $C(X)$ é $w^{*}$-denso em $X^{* *}$.

(vide [26, p. 233])

A seguir, veremos a definição de duas propriedades relacionadas às propriedades DP e DP1.

Definição 1.1.44. Um espaço de Banach tem a propriedade de Schur se as sequências wconvergentes e convergentes coincidem.

Teorema 1.1.45. O espaço $\ell_{1}$ tem a propriedade de Schur.

(vide [26, p. 218])

Exemplo 1.1.46. $\mathrm{O}$ espaço $\ell_{2}$ não tem a propriedade de Schur.

(vide [26, p. 212])

A seguir, definimos uma versão mais fraca da propriedade de Schur.

Definição 1.1.47. Um espaço de Banach $X$ tem a propriedade Kadec-Klee se dados $\left(x_{n}\right)_{n} \subset X$ e $x \in X$, com $\left\|x_{n}\right\|=\|x\|=1$, para todo $n \in \mathbb{N}$, e $x_{n} \stackrel{w}{\longrightarrow} x$ em $X$, temos que $\left\|x_{n}-x\right\| \longrightarrow 0$.

Essa propriedade também é conhecida como propriedade de Radon-Riesz, pois J. Radon e F. Riesz provaram, em 1913 e 1928, respectivamente, que os espaços $L_{p}(\Omega, \Sigma, \mu)$, sendo $\mu$ uma medida positiva em uma $\sigma$-álgebra $\Sigma$ de subconjuntos do conjunto $\Omega$, possuem essa propriedade quando $1<p<\infty$. Em 1958 e 1960, M. I. Kadec e V. L. Klee usaram versões dessa propriedade para desenvolver pré-requisitos para a demonstração de que os espaços de Banach separáveis de dimensão infinita são homeomorfos. Assim, essa propriedade é citada por alguns de Kadec-Klee.

Nesta dissertação, usaremos KKP para denotar a propriedade Kadec-Klee.

Exemplo 1.1.48. $\mathrm{O}$ espaço $\ell_{2}$ tem a propriedade KKP.

(vide [26, p. 220]) 
Por fim, operadores $w$-compactos serão definidos.

Definição 1.1.49. Sejam $X$ e $Y$ espaços de Banach. Um operador linear $T: X \rightarrow Y$ é denominado $\boldsymbol{w}$-compacto se, para todo subconjunto limitado $W$ de $X$, temos que $T(W)$ é w-relativamente compacto em $Y$, ou seja, $\overline{T(W)}^{w}$ é compacto em $Y$.

O espaço de todas os operadores lineares $w$-compactos de $X$ em $Y$ será denotado por $K_{w}(X, Y)$.

Proposição 1.1.50. Sejam $X$ e $Y$ espaços de Banach. Se um operador linear $T: X \rightarrow Y$ é w-compacto, então $T$ é contínuo.

(vide [26, p. 340])

Proposição 1.1.51. Sejam X, Y e Z espaços de Banach. Então:

(a) Se $X$ ou $Y$ é reflexivo, então todo operador em $L(X, Y)$ é w-compacto;

(b) Se $T: X \rightarrow Y$ é w-compacto e $S \in L(Y, Z)$, então $S \circ T$ éw-compacto;

(c) Se $T: X \rightarrow Y$ é w-compacto e $S \in L(Z, X)$, então $T \circ S$ é w-compacto.

(vide [26, p. 340 e 342])

Teorema 1.1.52 (Gantmacher). Sejam $X, Y$ espaços de Banach e $T \in L(X, Y)$. Então, $T$ é $w$-compacto se, e somente se, $T^{*}$ é w-compacto.

(vide [26, p. 343])

Teorema 1.1.53. Sejam $X$ e $Z$ espaços de Banach. Se $T: X \rightarrow Z$ é um operador $w$-compacto, então existem $Y$ um espaço reflexivo, $S \in L(X, Y)$ e $R \in L(Y, Z)$ tais que $T=R \circ S$.

(vide Corolário 1 em [15])

Exemplo 1.1.54. Seja $c_{0}$ o espaço vetorial formado por todas as sequências de elementos em $\mathbb{K}$ que convergem para zero. O espaço $c_{0}$ é um espaço de Banach munido da norma do supremo. Sejam $X$ um espaço de Banach e $\left(f_{n}\right)_{n}$ uma sequência $w$-convergente para zero em $X^{*}$. Vamos definir $T \in L\left(X, c_{0}\right)$ por $T(x)=\left(f_{n}(x)\right)_{n}$, para todo $x \in X$. Assim, $T$ é operador linear, contínuo e $w$-compacto.

(vide $[24$, p. 12])

\subsection{Base de Schauder}

Nesta seção, daremos algumas definições e resultados sobre bases de Schauder. Além disso, desenvolveremos alguns exemplos, que serão úteis neste trabalho. A referência utilizada foi [26].

Definição 1.2.1. Seja $X$ um espaço de Banach. Uma base de Schauder de $X$ é uma sequência $\left(x_{n}\right)_{n}$ de vetores de $X$ tal que, para todo $x \in X$, existe uma única sequência $\left(a_{n}\right)_{n}$ de escalares reais tal que $x=\sum_{n=1}^{\infty} a_{n} x_{n}$.

Exemplo 1.2.2. Se $X=c_{0}$ ou $\ell_{p}$, com $1 \leq p<\infty$, é fácil ver que a sequência $\left(e_{n}\right)_{n}$ é uma base de Schauder de $X$ e que $\left(a_{n}\right)=\sum_{n=1}^{\infty} a_{n} e_{n}$, para todo $\left(a_{n}\right) \in X$.

Porém, a sequência $\left(e_{n}\right)_{n}$ não é uma base para $\ell_{\infty}$. Tome, por exemplo, a sequência $(1,1, \ldots) \in$ $\ell_{\infty}$, segue que não existe uma sequência $\left(a_{n}\right)_{n}$ de escalares tal que $(1,1, \ldots)=\sum_{n=1}^{\infty} a_{n} e_{n}$. 
As projeções canônicas são de grande valia na Seção 3.1, na qual tais projeções nos permitirão aproximar os operadores compactos.

Proposição 1.2.3. Sejam X um espaço de Banach, $\left(x_{n}\right)_{n}$ uma base de Schauder de Xe $x \in X$ tal que $x=\sum_{n=1}^{\infty} a_{n} x_{n}$.

(a) Para todo $m \in \mathbb{N}$, considere $P_{m}(x)=\sum_{n=1}^{m} a_{n} x_{n}$ a projeção canônica, então $P_{m}$ é linear, continua e $\sup _{m \in \mathbb{N}}\left\|P_{m}\right\|<\infty$;

(b) Para todo $m \in \mathbb{N}$, considere $\psi_{m}(x)=a_{m}$ o funcional coeficiente de $\left(x_{n}\right)_{n}$, então $\psi_{m}$ é contínuo.

(vide [26, p. 354])

Definição 1.2.4. Sejam $X$ um espaço de Banach, $\left(x_{n}\right)_{n}$ uma base de Schauder de $X$ e $P_{n}$ as projeçôes canônicas associadas a $\left(x_{n}\right)_{n}$. A constante $k=\sup _{n \in \mathbb{N}}\left\|P_{n}\right\|$ chama-se constante básica associada à base $\left(x_{n}\right)_{n}$.

Definição 1.2.5. Sejam $X$ um espaço de Banach e $\left(x_{n}\right)_{n}$ uma base de Schauder de $X$. Para cada $m \in \mathbb{N}$ e $f \in X^{*}$, considere $\|f\|_{(m)}$ a norma da restrição de $f$ em $\overline{\left[x_{n}: n>m\right]}$. Dizemos que $\left(x_{n}\right)_{n}$ é uma base contrátil se $\lim _{m \rightarrow \infty}\|f\|_{(m)}=0$, para cada $f \in X^{*}$.

Proposição 1.2.6. Sejam $X$ um espaço de Banach, $\left(x_{n}\right)_{n}$ uma base de Schauder de $X$ e $\left(\psi_{n}\right)_{n}$ a sequência de funcionais coeficientes de $\left(x_{n}\right)_{n}$. Então, $\left(\psi_{n}\right)_{n}$ é base de $X^{*}$ se, e somente se, $\left(x_{n}\right)_{n}$ é contrátil.

(vide [26, p. 403])

O conceito de base contrátil será de suma importância na Seção 3.1, uma vez que precisaremos que não só o espaço de Banach $X$ tenha base de Schauder, mas também o seu dual $X^{*}$.

Exemplo 1.2.7. Se $X=c_{0}$ ou $\ell_{p}$, com $1<p<\infty$, e $\left(e_{n}\right)_{n}$ a base canônica de $X$, então a identificação natural de $X^{*} \operatorname{com} \ell_{q}$, com $\frac{1}{p}+\frac{1}{q}=1$, mostra que $\left(e_{n}\right)_{n}$ é contrátil.

Porém, $\left(e_{n}\right)$ não é uma base contrátil de $\ell_{1}$, uma vez que $l_{1}^{*} \cong l_{\infty}$. De fato, considere $f \in \ell_{1}^{*}$ identificado com o elemento $(1,1, \cdots) \in \ell_{\infty}$, então $\left\|x^{*}\right\|_{(m)}=1$, para todo $m \in \mathbb{N}$.

É sabido que um espaço de Banach com base de Schauder $X$ é reflexivo se, e somente se, toda base de Schauder de $X$ é contrátil e completamente limitada (vide [26, p. 406]). Em [33] Zippin provou um resultado mais forte que enunciamos a seguir.

Teorema 1.2.8. Seja $X$ um espaço de Banach com base de Schauder. Então, $X$ é reflexivo se, e somente se, toda base de $X$ é contrátil.

(vide [33])

\subsection{Resultados em Espaços de Hilbert}

Enunciaremos alguns resultados aplicáveis aos espaços de Hilbert, que terão visibilidade no que será discutido na Seção 3.2. Como referências utilizamos os livros [7] e [14]. 
Teorema 1.3.1. Sejam $H$ um espaço de Hilbert e $M$ um subespaço fechado de $H$. Então

(a) $H=M \oplus M^{\perp}$, isto é, cada $x \in H$ admite uma única representação na forma $x=p+q$ com $p \in M$ e $q \in M^{\perp}$. Além disso, $\|x-p\|=\operatorname{dist}(x, M)$. O vetor $p$ é chamado de projeção ortogonal de $x$ sobre $M$;

(b) Os operadores $P, Q: H \longrightarrow H$ dados por $P(x)=p$ e $Q(x)=q$ são projeções, isto é, são lineares, continuas e $P^{2}=P$ e $Q^{2}=Q$. Mais ainda, $P(H)=M, Q(H)=M^{\perp},\|P\|=1$ se $M \neq\{0\}$ e $\|Q\|=1$ se $M \neq H$. O operador $P$ é chamado de projeção ortogonal de $H$ sobre $M$;

(c) $P Q=Q P=0$.

(vide [7, p. 111])

Teorema 1.3.2 (Desigualdade de Bessel). Seja $S=\left\{x_{i}: i \in I\right\}$ um conjunto ortonormal no espaço de Hilbert H. Então, para todo $x \in H$

$$
\sum_{i \in J}\left|\left\langle x, x_{i}\right\rangle\right|^{2} \leq\|x\|^{2}
$$

sendo $J=\left\{i \in I:\left\langle x, x_{i}\right\rangle \neq 0\right\}$.

(vide [7, p. 116])

Proposição 1.3.3. Sejam $H_{1}, \ldots, H_{n}$ espaços de Hilbert e $H=H_{1} \oplus \cdots \oplus H_{n}$. Definimos

$$
\langle x, y\rangle=\sum_{i=1}^{n}\left\langle x_{i}, y_{i}\right\rangle,
$$

para todos $x, y \in H$, sendo $x=\left(x_{n}\right)$ e $y=\left(y_{n}\right)$. Então, $\langle\cdot, \cdot\rangle$ é um produto interno em $H e$

$$
\|x\|=\left(\sum_{i=1}^{n}\left\|x_{i}\right\|^{2}\right)^{1 / 2}
$$

é a norma associada ao produto interno. Com este produto interno, $H$ é um espaço de Hilbert.

(vide [14, p. 24])

A seguir, os funcionais lineares contínuos em um espaço de Hilbert serão estudados.

Exemplo 1.3.4. Seja $H$ um espaço de Hilbert. Para cada $y \in H$, o funcional

$$
\begin{aligned}
\phi_{y}: H & \longrightarrow \mathbb{K} \\
x & \longrightarrow \phi_{y}(x)=\langle x, y\rangle
\end{aligned}
$$

é claramente linear. Da desigualdade de Cauchy-Schwarz, resulta que $\phi_{y}$ é contínuo e que $\left\|\phi_{y}\right\| \leq$ $\|y\|$. Para $y=0$ é imediato que $\left\|\phi_{y}\right\|=\|y\|$. Para $y \neq 0$, observemos que

$$
\left|\phi_{y}\left(\frac{y}{\|y\|}\right)\right|=\frac{\left|\phi_{y}(y)\right|}{\|y\|}=\|y\| .
$$

Em resumo, $\phi_{y} \in H^{*}$ e $\left\|\phi_{y}\right\|=\|y\|$. 
O próximo resultado garante que todos os funcionais lineares contínuos em um espaço de Hilbert são da forma descrita no exemplo anterior.

Teorema 1.3.5 (Riesz-Fréchet). Sejam $H$ um espaço de Hilbert e $\varphi: H \longrightarrow \mathbb{K}$ um funcional linear contínuo. Então existe um único $y_{0} \in H$ tal que $\varphi(x)=\left\langle x, y_{0}\right\rangle$ para todo $x \in H$. Além disso, $\|\varphi\|=\left\|y_{0}\right\|$.

(vide [7, p. 126])

Teorema 1.3.6. Todo espaço de Hilbert é reflexivo.

(vide [7, p. 128])

Definição 1.3.7. Sejam $H$ um espaço de Hilbert e $T \in L(H)$. Dizemos que $T$ é operador positivo se $\langle T(h), h\rangle \geq 0$, para todo $h \in H$.

(vide [14, p. 57])

O operador transposto será muito utilizado na Seção 3.2. A seguir, definimos tal operador e enunciamos algumas de suas propriedades.

Teorema 1.3.8. Sejam $H_{1}$ e $H_{2}$ espaços de Hilbert. Para cada $T \in L\left(H_{1}, H_{2}\right)$, existe um único $T^{t} \in L\left(H_{2}, H_{1}\right)$ tal que $\langle T(x), y\rangle=\left\langle x, T^{t}(y)\right\rangle$, para todo $x \in H_{1}$ e todo $y \in H_{2}$. Além disso, $\|T\|=\left\|T^{t}\right\|$, e se $T$ é compacto, então $T^{t}$ também é compacto.

(vide [14, p. 31])

Proposição 1.3.9. Sejam $H$ espaço de Hilbert, $T, S \in L(H)$ e $\alpha \in \mathbb{K}$. Então:

(a) $(T+S)^{t}=T^{t}+S^{t}$;

(b) $(\alpha T)^{t}=\bar{\alpha} T^{t}$;

(c) $(T S)^{t}=S^{t} T^{t}$;

(d) $\left(T^{t}\right)^{t}=T$.

(vide [14, p. 32])

O operador $T^{t}$ é chamado de operador transposto de $T$. É importante destacar que esse operador também é conhecido como operador adjunto de Hilbert, com a notação $T^{*}$. Para não causar confusão com o adjunto entre espaços duais (Definição 1.1.11), optamos por operador transposto e usaremos a notação $T^{t}$. Como pode ser visto em [14, p. 167], os operadores $T^{t}$ e $T^{*}$ se relacionam por meio de duas aplicações conjugadas-lineares.

\section{4 Álgebras de Banach}

Nesta seção, introduziremos o conceito de álgebras de Banach e seus principais exemplos. Esses conceitos serão usados na Seção 3.3, na qual obtemos uma caracterização da DP1 para subálgebras das álgebras $K(X)$ e $K(H)$. 
Definição 1.4.1. Dado A um espaço vetorial sobre um corpo $\mathbb{K}$ dizemos que $A$ é uma álgebra se existir uma aplicação bilinear

$$
\begin{aligned}
A \times A & \longrightarrow A \\
(a, b) & \longmapsto a b
\end{aligned}
$$

tal que, para $a, b, c \in A$ temos que

$$
a(b c)=(a b) c .
$$

Essa aplicação é denominada multiplicação.

Dizemos que $A$ tem unidade se existe $1_{A} \in A$ que satisfaz $a 1_{A} a=a 1_{A}=a$, para todo $a \in A$.

Se $B$ é subespaço vetorial de $A$ tal que $b_{1} b_{2} \in B$, para todo $b_{1}, b_{2} \in B$, então $B$ munido da multiplicação de A restrita a B, é uma subálgebra de $A$.

Definição 1.4.2. Seja A uma álgebra. Se A possui uma norma sub-multiplicativa $\|\cdot\|$, ou seja, que satisfaz à desigualdade

$$
\|a b\| \leq\|a\|\|b\|,
$$

para todo $a, b \in A$, então o par $(A,\|\cdot\|)$ é chamado álgebra normada.

Além disso, se A for completo na norma $\|\cdot\|$, dizemos que A é uma álgebra de Banach.

Seja $A$ uma álgebra normada. Segue da definição que para $a, b, c, d \in A$,

$$
\|a b-c d\|=\|a b-a d+a d-c d\| \leq\|a\|\|b-d\|+\|a-c\|\|d\|,
$$

de onde decorre a continuidade da multiplicação na álgebra normada $A$.

A seguir, vejamos alguns exemplos de álgebras de Banach.

Exemplo 1.4.3. Seja $K$ um espaço topológico Hausdorff localmente compacto. Seja $C_{0}(K)$ o conjunto das funções $f: K \longrightarrow \mathbb{C}$ que se anulam no infinito, ou seja, para todo $\varepsilon>0$ o conjunto $\{x \in K:|f(x)| \geq \varepsilon\}$ é compacto.

Consideremos as operações usuais de soma e multiplicação por escalar que tornam $C_{0}(K)$ um espaço vetorial sobre o corpo $\mathbb{C}$, e a operação usual de multiplicação que faz de $C_{0}(K)$ uma álgebra.

A norma do supremo

$$
\|f\|_{\infty}=\sup _{x \in K}|f(x)|
$$

é uma norma sub-multiplicativa e completa.

Temos que $C_{0}(K)$, munido dessas operações, é uma álgebra de Banach.

Exemplo 1.4.4. Seja $K$ um espaço topológico Hausdorff compacto, então $C_{0}(K)=C(K)=\{f$ : $K \longrightarrow \mathbb{C}: f$ é contínua\}. De fato, para toda $f \in C(K)$ e todo $\varepsilon>0$, temos que o conjunto $\{x \in K:|f(x)| \geq \varepsilon\}$ é compacto, pois é um subconjunto fechado de $K$. Temos que $C(K)$ é uma álgebra de Banach com unidade. 
Exemplo 1.4.5. Seja $X$ um espaço de Banach. O espaço $L(X)$ é uma álgebra de Banach não comutativa com unidade, sendo que a operação multiplicação é dada pela composição e a unidade é o operador identidade $I d_{X}$.

Exemplo 1.4.6. Seja $X$ um espaço de Banach. O espaço $K(X)$ é uma subálgebra de Banach de $L(X)$ sem unidade se $\operatorname{dim} X=\infty$.

A seguir, definiremos o espectro e o raio espectral de uma álgebra.

Definição 1.4.7. Sejam A uma álgebra com unidade 1 e $a \in A$. Definimos o espectro de a como sendo o conjunto

$$
\sigma(a)=\{\lambda \in \mathbb{C}: a-\lambda 1 \text { não é invertível em } A\} .
$$

Teorema 1.4.8. Sejam $A$ uma álgebra com unidade e $a \in A$. Se $\sigma(a)$ é não vazio e q é um polinômio na álgebra dos polinômios com coeficientes complexos, então $\sigma(q(a))=q(\sigma(a))$, sendo $q(\sigma(a))=\{q(\lambda) \in \mathbb{C}: \lambda \in \sigma(a)\}$.

(vide $[28, p .7])$

Definição 1.4.9. Sejam A uma álgebra de Banach com unidade e a $\in$ A. Definimos o raio espectral de a por

$$
r(a)=\sup _{\lambda \in \sigma(a)}|\lambda|
$$

Encerramos com a definição de ideal em uma álgebra.

Definição 1.4.10. Um ideal à esquerda em uma álgebra $A$ é um subespaço vetorial $I$ de $A$ tal que, se $a \in A$ e $b \in I$, então $a b \in I$.

Um ideal à direita em uma álgebra $A$ é um subespaço vetorial I de A tal que, se $a \in A$ e $b \in I$, então $b a \in I$.

Um ideal em uma álgebra $A$ é um subespaço vetorial que é, ao mesmo tempo, ideal à esquerda e à direita de $A$.

Claramente, $\{0\}$ e $A$ são ideais em $A$ chamados de ideais triviais.

Exemplo 1.4.11. Se $X$ é um espaço de Banach, então $K(X)$ é um ideal de $L(X)$. 


\section{Capítulo 2}

\section{Propriedade Dunford-Pettis Alternativa}

Neste capítulo, trataremos da Propriedade Dunford-Pettis Alternativa - ao longo do texto, usaremos propriedade DP1 por brevidade. Estudaremos resultados equivalentes à definição da propriedade DP1; reproduziremos exemplos de espaços que possuam (ou não) tal propriedade; e apresentaremos resultados que contribuem para a sua caracterização e são úteis no desenvolvimento do Capítulo 3. Entretanto, antes de estudar essa propriedade, torna-se necessário estudar a propriedade de Dunford-Pettis - ou apenas propriedade $D P$-, uma vez que a definição da propriedade DP1 é inspirada na propriedade DP.

A propriedade DP será apresentada na próxima seção, com alguns resultados e exemplos. Esses serão importantes para relacionarmos as propriedades DP e DP1, e, na Seção 4.4, verificarmos a equivalência entre elas em $C^{*}$-álgebras.

\subsection{Propriedade Dunford-Pettis}

Em 1940, Nelson J. Dunford e Billy J. Pettis provaram que, para uma medida $\mu$ e um espaço de Banach $X$, as aplicações lineares $w$-compactas de $L_{1}(\mu)$ em $X$ são $w$-sequencialmente contínuas [17]. No entanto, somente em 1953 Grothendieck introduziu a definição de um espaço de Banach ter a propriedade de Dunford-Pettis e mostrou que todo operador $w$-compacto em $C(K)$ é $w$ sequencialmente contínuo [22].

A propriedade DP apresenta fortes consequências na teoria de geometria de espaços de Banach e foi amplamente estudada, por exemplo, nos artigos [9], [11], [12], [13] e [16]. Nosso intuito nesta seção é enunciar os resultados e exemplos relacionados à propriedade DP que serão necessários ao longo do texto. Omitiremos as demonstrações, as quais podem ser examinadas na dissertação de mestrado [24] de A. Hissadomi, pois, apesar de não serem os resultados originais, trata-se de um texto completo, detalhado e bem redigido sobre a propriedade DP.

Entre os resultados serão encontrados: (i) equivalências para a definição da propriedade DP; (ii) exemplos de espaços que possuem a propriedade DP, como os espaços $\ell_{1}, c_{0}, \ell_{\infty}$ e $C(K)$, em que $K$ é um compacto; (iii) exemplos de espaços que não possuem a DP, como os espaços $\ell_{p}$, com $1<p<\infty$; (iv) a propriedade DP é preservada por subespaço complementado; e (v) se o dual de um espaço de Banach $X^{*}$ tem a propriedade DP, então $X$ tem a propriedade DP. A recíproca, no entanto, não é válida - o contra-exemplo foi dado por Stegall em [31]. 
Definição 2.1.1. Dizemos que um espaço de Banach $X$ tem propriedade Dunford-Pettis se, dados qualquer espaço de Banach $Y$ e qualquer aplicação linear w-compacta $T: X \rightarrow Y, T$ aplica sequências w-Cauchy em $X$ em sequências de Cauchy em $Y$.

O seguinte teorema nos dá várias equivalências importantes para a definição da propriedade DP.

Teorema 2.1.2. Seja $X$ um espaço de Banach. As seguintes condições são equivalentes:

(a) X tem a propriedade DP;

(b) dados qualquer espaço de Banach $Y$ e qualquer aplicação linear w-compacta $T: X \rightarrow Y, T$ aplica sequências w-Cauchy em sequências covergentes;

(c) dados qualquer espaço de Banach $Y$ e qualquer aplicação linear w-compacta $T: X \rightarrow Y, T$ aplica sequências $w$-convergentes a zero em sequências convergentes a zero, isto é, qualquer aplicação w-compacta é w-sequencialmente contínua;

(d) a condição (c) é satisfeita para $Y=c_{0}$;

(e) se $\left(x_{n}\right)_{n}$ e $\left(f_{n}\right)_{n}$ são sequências $w$-convergentes a zero em $X$ e $X^{*}$ respectivamente, então $\lim _{n \rightarrow \infty} f_{n}\left(x_{n}\right)=0$;

(f) se $\left(x_{n}\right)_{n}$ é uma sequência $w$-Cauchy em $X$ e $\left(f_{n}\right)_{n}$ é uma sequência w-convergente a zero em $X^{*}$, então $\lim _{n \rightarrow \infty} f_{n}\left(x_{n}\right)=0$.

(vide [24, p. 60])

A seguir, veremos alguns corolários, a partir dos quais obteremos exemplos de espaços que possuem (ou não) a propriedade DP.

Corolário 2.1.3. Um espaço de Banach reflexivo $X$ tem a propriedade $D P$ se, e somente se, $\operatorname{dim} X<\infty$.

(vide [24, p. 63])

Como consequência imediata do corolário anterior, temos o seguinte exemplo:

Exemplo 2.1.4. Seja $X=\ell_{p}$, para $1<p<\infty$. Como $X$ é reflexivo e $\operatorname{dim} X=\infty, X$ não tem a propriedade DP.

A propriedade de Schur foi definida em 1.1.44. Este corolário relaciona a propriedade de Schur com a propriedade DP.

Corolário 2.1.5. Se $X$ é um espaço de Banach com a propriedade de Schur, então $X$ tem a propriedade DP.

(vide [24, p. 64])

Exemplo 2.1.6. Pelo Teorema 1.1.45, sabemos que o espaço $\ell_{1}$ tem a propriedade de Schur, então segue, do corolário acima, que $\ell_{1}$ tem a propriedade DP. 
A propriedade DP é preservada por subespaço complementado:

Proposição 2.1.7. Seja $X$ um espaço de Banach com a propriedade DP. Então todo subespaço complementado de $X$ tem a propriedade DP.

(vide [24, p. 65])

Relacionaremos um espaço de Banach com o seu dual a partir da propriedade DP.

Proposição 2.1.8. Seja $X$ um espaço de Banach. Se $X^{*}$ tem a propriedade DP, então $X$ também tem a propriedade DP.

(vide [24, p. 64])

Exemplo 2.1.9. Seja $X=c_{0}$. Como $c_{0}^{*} \cong \ell_{1}$, segue que $X^{*}$ tem a propriedade DP. Assim, usando o corolário acima, temos que $c_{0}$ tem a propriedade DP.

É importante notar que a recíproca da Proposição 2.1.8 não vale. O exemplo é o espaço dado por Stegall em [31], apresentado a seguir.

Exemplo 2.1.10. Para cada $n \in \mathbb{N}$, denotaremos por $\ell_{2}^{(n)}$ o espaço vetorial $\mathbb{K}^{n}$ com a norma de $\ell_{2}$. Seja $X=\left(\oplus_{n=1}^{\infty} \ell_{2}^{(n)}\right)_{1}$, isto é, cada $x \in X$ é tal que $x=\left(x^{(n)}\right)_{n}$, em que $x^{(n)} \in \ell_{2}^{(n)}$ e $\|x\|=\sum_{n=1}^{\infty}\left\|x^{(n)}\right\|_{2}=\sum_{n=1}^{\infty}\left(\sum_{i=1}^{n}\left|x_{i}^{(n)}\right|^{2}\right)^{\frac{1}{2}}$. Temos que $X^{*}=\left(\oplus_{n=1}^{\infty} \ell_{2}^{(n)}\right)_{\infty}$. Além disso, $X$ é um espaço de Banach e tem a propriedade DP, mas $X^{*}$ não tem a propriedade DP.

(vide [24, p. 65])

Outros exemplos de espaços que possuem a propriedade DP:

Exemplo 2.1.11. Todo subespaço fechado de $c_{0}$ tem a propriedade DP. (vide $[24$, p. 70])

Exemplo 2.1.12. O espaço $C(K)$, em que $K$ é um compacto Hausdorff, tem a propriedade DP. (vide [24, p. 75])

Exemplo 2.1.13. $\ell_{\infty}$ tem a propriedade DP.

(vide [24, p. 75])

Como antecipado, os espaços $L_{1}(\mu)$ e $C(K)$ possuem a DP, sendo $\mu$ uma medida e $K$ um compacto Hausdorff. A partir desses resultados, é possível mostrar que toda álgebra de von Neumann (a ser definida na Seção 4.3) comutativa e seu pré-dual possuem a DP. Em geral, no caso não comutativo, isso não é verdade. Bunce mostrou, em [9], que o pré-dual de uma álgebra de von Neumann possui a DP se, e somente se, a álgebra de von Neumann satisfaz determinadas condições.

\subsection{Propriedade Dunford-Pettis Alternativa}

Freedman, em [21], definiu a propriedade Dunford-Pettis alternativa como a restrição da propriedade Dunford-Pettis à esfera unitária. Se observarmos a relação entre as propriedades de Schur e Kadec-Klee, definidas na Seção 1.1 (Definições 1.1.44 e 1.1.47), a propriedade Kadec-Klee é mais fraca que a propriedade de Schur, uma vez que a propriedade Kadec-Klee tem como hipótese a 
sequência estar na esfera unitária. Assim vemos que a propriedade DP1 foi definida de maneira análoga.

Já observamos que nem todo pré-dual de uma álgebra de von Neumann comutativa tem a propriedade DP. Ao definir a propriedade Dunford-Pettis alternativa, Freedman estava em busca de que mais pré-duais de álgebras de von Neumann não comutativas pudessem ter tal propriedade. Isso foi alcançado no artigo supracitado: ele mostrou um exemplo de uma álgebra de von Neumann não comutativa tal que o seu pré-dual possui a DP1, mas não possui a DP.

Ao final do mesmo artigo, Freedman conjecturou que o pré-dual de toda álgebra de von Neumann poderia ter a DP1. Entretanto, em [25], Martín e Peralta mostraram a existência de álgebras de von Neumann não comutativas tais que os pré-duais não possuam a propriedade DP1. Freedman também conjecturou que as propriedades DP e DP1 poderiam ser equivalentes em $C^{*}$-álgebras. Posteriormente, em [10], Bunce e Peralta mostraram essa equivalência. Apresentaremos esse resultado na Seção 4.4.

Iniciaremos esta seção pelo estudo da definição da propriedade DP1 e de relações entre as propriedades DP, DP1 e KKP. Posteriormente, veremos a definição de um operador DP1, o que nos trará algumas resultados equivalentes à definição da propriedade DP1. Essa caracterização por meio dos operadores DP1 será utilizada no Capítulo 3. É importante destacar que os operadores DP1 desempenham um papel relevante no estudo da propriedade DP1 - papel análogo aos operadores $w$-sequencialmente contínuos em relação à propriedade DP.

Estudaremos exemplos de espaços que possuem a DP1, entre os quais estão os espaços de Hilbert e os espaços $\ell_{p}$, com $1 \leq p \leq \infty$. Por outro lado, o espaço $\ell_{2} \oplus_{\infty} \ell_{2}$ não possui a DP1. Por meio desses exemplos, veremos que a propriedade DP1 é estritamente mais fraca que a propriedade DP em espaços de Banach em geral. Por fim, verificaremos que a propriedade DP1 é preservada por subespaços complementados, isomorfismo isométrico, soma direta com a norma $p$, com $1 \leq p<\infty$, e espaço quociente (sob algumas condições sobre o espaço).

Definição 2.2.1. Um espaço de Banach $X$ tem a propriedade Dunford-Pettis alternativa se, dados $\left(x_{n}\right)_{n} \subset X$ e $x \in X$ tais que $\left\|x_{n}\right\|=\|x\|=1$, para todo $n \in \mathbb{N}$, e $x_{n} \stackrel{w}{\longrightarrow} x$ em $X$, e dados $\left(f_{n}\right)_{n} \subset X^{*}$ e $f \in X^{*}$, com $f_{n} \stackrel{w}{\longrightarrow} f$ em $X^{*}$, temos que $f_{n}\left(x_{n}\right) \longrightarrow f(x)$.

Observação 2.2.2. Podemos supor, sem perda de generalidade, que $f=0$. Basta trocarmos $f_{n}$ por $f_{n}-f$.

A próxima proposição nos dá uma equivalência para a propriedade DP1, na qual a condição $\left\|x_{n}\right\|=\|x\|=1$ será substituída pela condição equivalente $\left\|x_{n}\right\| \longrightarrow\|x\|$.

Proposição 2.2.3. Seja $X$ um espaço de Banach. Então, $X$ tem a propriedade DP1 se, e somente se, para toda sequência $x_{n} \stackrel{w}{\longrightarrow} x$ em $X$, com $\left\|x_{n}\right\| \longrightarrow\|x\|$, e toda sequência $f_{n} \stackrel{w}{\longrightarrow} 0$ em $X^{*}$, temos que $f_{n}\left(x_{n}\right) \longrightarrow 0$.

Demonstração. $(\Longrightarrow)$ Sejam $X$ um espaço de Banach com a propriedade DP1, $x_{n} \stackrel{w}{\longrightarrow} x$ em $X$, com $\left\|x_{n}\right\| \longrightarrow\|x\|$, e $f_{n} \stackrel{w}{\longrightarrow} 0$ em $X^{*}$. Para mostrar que $f_{n}\left(x_{n}\right) \longrightarrow 0$, dividiremos em dois casos:

Caso (i): Suponha que $x \neq 0$.

Segue que $\|x\| \neq 0$ e existe $m \in \mathbb{N}$ tal que $\left\|x_{n}\right\| \neq 0$, para todo $n \geq m$. Assim:

$$
\left\|\frac{x_{n}}{\left\|x_{n}\right\|}\right\|=\left\|\frac{x}{\|x\|}\right\|=1 \quad \text { e } \quad \frac{x_{n}}{\left\|x_{n}\right\|} \stackrel{w}{\longrightarrow} \frac{x}{\|x\|} .
$$


Como $X$ tem a DP1, segue que

$$
\frac{1}{\left\|x_{n}\right\|} f_{n}\left(x_{n}\right)=f_{n}\left(\frac{x_{n}}{\left\|x_{n}\right\|}\right) \longrightarrow 0 .
$$

Mas $\left\|x_{n}\right\| \longrightarrow\|x\|$, então $f_{n}\left(x_{n}\right) \longrightarrow 0$.

Caso (ii): Suponha que $x=0$.

Tomemos $y \in X$ tal que $\|y\|=1$. Como $\left\|x_{n}\right\| \longrightarrow 0$, segue que $\left\|x_{n}+y\right\| \longrightarrow\|y\|$. E, como $x_{n} \stackrel{w}{\longrightarrow} 0$ em $X$, então $x_{n}+y \stackrel{w}{\longrightarrow} y$.

Pelo caso (i), segue que $f_{n}\left(x_{n}+y\right) \longrightarrow 0$. Como $f_{n} \stackrel{w}{\longrightarrow} 0$, então $f_{n}(y) \longrightarrow 0$. Logo, $f_{n}\left(x_{n}\right) \longrightarrow 0$.

$(\Longleftarrow)$ É imediata.

A seguir serão estudadas relações entre as propriedades Dunford-Pettis, Dunford-Pettis alternativa e Kadec-Klee.

Proposição 2.2.4. Sejam X e $Y$ espaços de Banach. Então:

(a) Se $X$ tem a propriedade DP, então $X$ tem a propriedade DP1;

(b) Se $X$ tem a propriedade KKP, então $X$ tem a propriedade DP1;

(c) Se X tem a propriedade DP1 e X, Y são isometricamente isomorfos, então $Y$ tem a propriedade DP1;

(d) Se $X$ tem a propriedade KKP e X, Y são isometricamente isomorfos, então $Y$ tem a propriedade KKP.

Demonstração. (a) Sejam $x_{n} \stackrel{w}{\longrightarrow} x$ em $X$, com $\left\|x_{n}\right\|=\|x\|=1$, e $f_{n} \stackrel{w}{\longrightarrow} 0$ em $X^{*}$. Então, $\left(x_{n}\right)_{n}$ é uma sequência $w$-Cauchy e, como $X$ tem a DP, segue do Teorema 2.1.2 que $f_{n}\left(x_{n}\right) \longrightarrow 0$. Logo, $X$ tem a propriedade DP1.

(b) Sejam $x_{n} \stackrel{w}{\longrightarrow} x$ em $X$, com $\left\|x_{n}\right\|=\|x\|=1$, e $f_{n} \stackrel{w}{\longrightarrow} f$ em $X^{*}$. Por hipótese, $X$ tem a propriedade KKP, então $\left\|x_{n}-x\right\| \longrightarrow 0$.

Como $f_{n} \stackrel{w}{\longrightarrow} f$, então existe $c$ tal que $\left\|f_{n}\right\| \leq c$ e $f_{n}(x) \longrightarrow f(x)$.

Dado $\varepsilon>0$, existe $n_{0} \in \mathbb{N}$ tal que, para todo $n \geq n_{0}$,

$$
\left\|x_{n}-x\right\|<\frac{\varepsilon}{2 c} \quad \text { e } \quad\left|f_{n}(x)-f(x)\right|<\frac{\varepsilon}{2} .
$$

Assim, para todo $n \geq n_{0}$, temos que

$$
\begin{aligned}
\left|f_{n}\left(x_{n}\right)-f(x)\right| & \leq\left|f_{n}\left(x_{n}\right)-f_{n}(x)\right|+\left|f_{n}(x)-f(x)\right| \\
& \leq\left\|f_{n}\right\|\left\|x_{n}-x\right\|+\left|f_{n}(x)-f(x)\right| \\
& <c \frac{\varepsilon}{2 c}+\frac{\varepsilon}{2}=\varepsilon .
\end{aligned}
$$

Logo, $f_{n}\left(x_{n}\right) \longrightarrow f(x)$ e, portanto, $X$ tem a propriedade DP1. 
(c) Sejam $T: X \rightarrow Y$ isomorfismo isométrico, $y_{n} \stackrel{w}{\longrightarrow} y$ em $Y$, com $\left\|y_{n}\right\|=\|y\|=1$, e $g_{n} \stackrel{w}{\longrightarrow} g$ em $Y^{*}$. Então, $T^{-1}\left(y_{n}\right) \stackrel{w}{\longrightarrow} T^{-1}(y)$ em $X,\left\|T^{-1}\left(y_{n}\right)\right\|=\left\|T^{-1}(y)\right\|=1$ e $T^{*} g_{n} \stackrel{w}{\longrightarrow} T^{*} g$ em $X^{*}$. Como $X$ tem a propriedade DP1, segue que $\left(T^{*} g_{n}\right)\left(T^{-1}\left(y_{n}\right)\right) \longrightarrow\left(T^{*} g\right)\left(T^{-1}(y)\right)$. Pela definição de adjunto, $g_{n}\left(y_{n}\right) \longrightarrow g(y)$. Logo, $Y$ tem a propriedade DP1.

(d) Sejam $T: X \rightarrow Y$ isomorfismo isométrico e $y_{n} \stackrel{w}{\longrightarrow} y$ em $Y$, com $\left\|y_{n}\right\|=\|y\|=1$. Então, $T^{-1}\left(y_{n}\right) \stackrel{w}{\longrightarrow} T^{-1}(y)$ em $X$ e $\left\|T^{-1}\left(y_{n}\right)\right\|=\left\|T^{-1}(y)\right\|=1$. Como $X$ tem a propriedade KKP, segue que $T^{-1}\left(y_{n}\right) \longrightarrow T^{-1}(y)$ em $X$. Assim, $T\left(T^{-1}\left(y_{n}\right)\right) \longrightarrow T\left(T^{-1}(y)\right)$ em $Y$, então $y_{n} \longrightarrow y$ em $Y$. Logo, $Y$ tem a propriedade KKP.

Como consequência do teorema anterior, temos o seguinte resultado:

Corolário 2.2.5. Todo espaço de Hilbert tem a propriedade DP1.

Demonstração. Seja $H$ um espaço de Hilbert. Pela Proposição 2.2.4 (b), basta mostrarmos que $H$ tem a propriedade KKP.

Seja $x_{n} \stackrel{w}{\longrightarrow} x$ em $X$, com $\left\|x_{n}\right\|=\|x\|=1$. Temos que

$$
\begin{aligned}
\left\|x_{n}-x\right\|^{2} & =\left\langle x_{n}-x, x_{n}-x\right\rangle=\left\langle x_{n}, x_{n}\right\rangle-\left\langle x_{n}, x\right\rangle-\left\langle x, x_{n}\right\rangle+\langle x, x\rangle \\
& =\left\|x_{n}\right\|^{2}+\|x\|^{2}-2 \operatorname{Re}\left(\left\langle x_{n}, x\right\rangle\right)=2\left(1-\operatorname{Re}\left(\left\langle x, x_{n}\right\rangle\right)\right) .
\end{aligned}
$$

Definimos $f(h)=\langle h, x\rangle$, para todo $h \in H$. Assim, $f$ é um operador linear contínuo em $H$ e $\|f\|=\|x\|=1$.

Como $x_{n} \stackrel{w}{\longrightarrow} x$, segue que $f\left(x_{n}\right) \longrightarrow f(x)$, isto é, $\left\langle x_{n}, x\right\rangle \longrightarrow\langle x, x\rangle=\|x\|=1$. Então, $\left\|x_{n}-x\right\| \longrightarrow 0$. Logo, $X$ tem a propriedade KKP e, portanto, tem propriedade DP1.

Em geral, a propriedade DP1 não implica a propriedade DP. Veremos isso no próximo exemplo.

Exemplo 2.2.6. $\mathrm{O}$ espaço $\ell_{2}$ não tem a propriedade DP, pois $\ell_{p}$, com $1<p<\infty$, não tem a propriedade DP (Exemplo 2.1.4). Como $\ell_{2}$ é um espaço de Hilbert, então tem a propriedade DP1. Assim, em geral, a propriedade DP1 não implica a propriedade DP.

Os operadores $w$-sequencialmente contínuos têm um papel importante no estudo da propriedade DP, como foi visto na Seção 2.1 brevemente. Definiremos os operadores DP1 que terão papel semelhante com relação à propriedade DP1.

Definição 2.2.7. Sejam $X$ e $Y$ espaços de Banach. Um operador $T \in L(X, Y)$ é um operador DP1 se, dados $\left(x_{n}\right)_{n} \subset X$ e $x \in X$ tais que $\left\|x_{n}\right\|=\|x\|=1$, para todo $n \in \mathbb{N}$, e $x_{n} \stackrel{w}{\longrightarrow} x$ em $X$, temos que $T\left(x_{n}\right) \longrightarrow T(x)$.

Proposição 2.2.8. Sejam $X, Y$ e $Z$ espaços de Banach. Se $T \in L(X, Y)$ é um operador DP1 e $S \in L(Y, Z)$, então $S \circ T$ é um operador DP1.

Demonstração. Seja $x_{n} \stackrel{w}{\longrightarrow} x$ em $X$, com $\left\|x_{n}\right\|=\|x\|=1$.

Como $T$ é um operador DP1, então $T\left(x_{n}\right) \longrightarrow T(x)$. Da continuidade de $S$, segue que $S\left(T\left(x_{n}\right)\right) \longrightarrow$ $S(T(x))$. Logo, $S \circ T$ é um operador DP1. 
De forma análoga ao Teorema 2.1.2 para a propriedade DP, o próximo teorema nos dará resultados equivalentes à definição da propriedade DP1. Essas equivalências serão usadas no Capítulo 3 .

Teorema 2.2.9. Seja $X$ um espaço de Banach. São equivalentes:

(a) X tem a propriedade DP1;

(b) Para todo $Y$ espaço de Banach, se $T: X \rightarrow Y$ é um operador $w$-compacto, então $T$ é um operador DP1;

(c) Para todo espaço $Y$ reflexivo, temos que todo operador $T: X \rightarrow Y$ é um operador DP1;

(d) Todo operador $T: X \rightarrow c_{0}$ w-compacto é um operador DP1;

(e) Seja $x_{n} \stackrel{w}{\longrightarrow} x$ em $X$, com $\left\|x_{n}\right\|=\|x\|=1$. Se $\left(f_{n}\right) \subseteq X^{*}$ é uma sequência $w$-Cauchy, então $f_{n}\left(x_{n}-x\right) \longrightarrow 0$.

Demonstração. (a) $\Rightarrow$ (b) Sejam $Y$ um espaço de Banach e $T: X \rightarrow Y$ um operador $w$-compacto. Suponha que $T$ não é um operador DP1, isto é, existe $x_{n} \stackrel{w}{\longrightarrow} x$ em $X$, com $\left\|x_{n}\right\|=\|x\|=1$, tal que $\left(T\left(x_{n}\right)\right)_{n}$ não converge em norma em $Y$. Então, $\left(T\left(x_{n}\right)\right)_{n}$ não é uma sequência de Cauchy em $Y$, pois $Y$ é um espaço de Banach. Assim, existem duas sequências de índices $\left\{n_{k}\right\}_{k=1}^{\infty},\left\{n_{k}^{\prime}\right\}_{k=1}^{\infty}$ e $\delta>0$ tais que

$$
\left\|T\left(x_{n_{k}}\right)-T\left(x_{n_{k}^{\prime}}\right)\right\|>\delta
$$

para todo $k \in \mathbb{N}$.

Pelo Teorema de Hahn-Banach (1.1.4), existe $g_{k} \in Y^{*}$ tal que $\left\|g_{k}\right\|=1$ e $g_{k}\left(T\left(x_{n_{k}}\right)-T\left(x_{n_{k}^{\prime}}\right)\right)=$ $\left\|T\left(x_{n_{k}}\right)-T\left(x_{n_{k}^{\prime}}\right)\right\|$. Então, para todo $k$, temos que

$$
\delta<\left|g_{k}\left(T\left(x_{n_{k}}\right)-T\left(x_{n_{k}^{\prime}}\right)\right)\right| .
$$

Definimos $f_{k}=T^{*}\left(g_{k}\right) \in T^{*}\left(B_{Y^{*}}\right) \subseteq X^{*}$, para todo $k$. Pelo Teorema 1.1.52, como $T$ é $w$ compacto, então $T^{*}$ é $w$-compacto, isto é, $T^{*}\left(B_{Y^{*}}\right)$ é $w$-relativamente compacto. Assim, pelo Teorema de Eberlein-Smulian (1.1.36), $\left(f_{k}\right)_{k}$ possui uma subsequência $w$-convergente. Então, sem perda de generalidade, existe $f \in X^{*}$ tal que $f_{k} \stackrel{w}{\longrightarrow} f$.

Mas, por hipótese, $X$ tem a propriedade DP1. Como $x_{n_{k}} \stackrel{w}{\longrightarrow} x$ e $x_{n_{k}^{\prime}} \stackrel{w}{\longrightarrow} x$, então $f_{k}\left(x_{n_{k}}\right) \longrightarrow$ $f(x)$ e $f_{k}\left(x_{n_{k}^{\prime}}\right) \longrightarrow f(x)$.

Assim, temos que

$$
\begin{aligned}
\delta & <\left|g_{k}\left(T\left(x_{n_{k}}\right)-T\left(x_{n_{k}^{\prime}}\right)\right)\right|=\left|g_{k}\left(T\left(x_{n_{k}}\right)\right)-g_{k}\left(T\left(x_{n_{k}^{\prime}}\right)\right)\right| \\
& =\left|T^{*}\left(g_{k}\right)\left(x_{n_{k}}\right)-T^{*}\left(g_{k}\right)\left(x_{n_{k}^{\prime}}\right)\right|=\left|f_{k}\left(x_{n_{k}}\right)-f_{k}\left(x_{n_{k}^{\prime}}\right)\right| \longrightarrow 0 .
\end{aligned}
$$

Absurdo. Logo, $T$ é um operador DP1.

(b) $\Rightarrow$ (c) Sejam $Y$ um espaço reflexivo e $T \in L(X, Y)$. Então, pela Proposição 1.1.51, $T$ é $w$-compacto. Assim, por hipótese, $T$ é um operador DP1. 
(c) $\Rightarrow$ (d) Seja $T: X \rightarrow c_{0}$ um operador $w$-compacto. Então, pelo Teorema 1.1.53, existem $Y$ um espaço reflexivo, $S \in L(X, Y)$ e $R \in L\left(Y, c_{0}\right)$ tal que $T=R \circ S$.

Seja $x_{n} \stackrel{w}{\longrightarrow} x$ em $X$, com $\left\|x_{n}\right\|=\|x\|=1$. Por hipótese, $S$ é um operador DP1, então, pela Proposição 2.2.8, $R \circ S$ é um operador DP1. Logo, $T$ é um operador DP1.

(d) $\Rightarrow$ (a) Sejam $x_{n} \stackrel{w}{\longrightarrow} x$ em $X$, com $\left\|x_{n}\right\|=\|x\|=1$, e $f_{n} \stackrel{w}{\longrightarrow} 0$ em $X^{*}$.

Definimos $T: X \rightarrow c_{0}$ dado por $T(x)=\left(f_{n}(x)\right)_{n}$, para todo $x \in X$. Assim, pelo Exemplo 1.1.54, temos que $T$ é um operador $w$-compacto. Por hipótese, $T$ é um operador DP1, ou seja, $T\left(x_{n}\right) \rightarrow T(x)$.

Para cada $n \in \mathbb{N}$, temos que

$$
\left\|T\left(x_{n}-x\right)\right\|=\left\|\left(f_{m}\left(x_{n}-x\right)\right)_{m=1}^{\infty}\right\|=\sup _{m \in \mathbb{N}}\left|f_{m}\left(x_{n}-x\right)\right| \geq\left|f_{n}\left(x_{n}-x\right)\right|
$$

e $\left(T\left(x_{n}-x\right)\right)_{n}$ converge para zero, então $f_{n}\left(x_{n}-x\right) \longrightarrow 0$. Como $f_{n} \stackrel{w}{\longrightarrow} 0$, segue que $f_{n}(x) \longrightarrow 0$. Logo, $f_{n}\left(x_{n}\right) \longrightarrow 0$ e, portanto, $X$ tem a propriedade DP1.

(a) $\Rightarrow$ (e) Sejam $x_{n} \stackrel{w}{\longrightarrow} x$ em $X$, com $\left\|x_{n}\right\|=\|x\|=1$, e $\left(f_{n}\right) \subset X^{*}$ uma sequência $w$-Cauchy. Fixemos $n \in \mathbb{N}$. Temos que $f_{n}\left(x_{k}\right) \longrightarrow f_{n}(x)$, quando $k \longrightarrow \infty$, pois $x_{k} \stackrel{w}{\longrightarrow} x$.

Assim, dado $\varepsilon>0$, existe $k_{n} \in \mathbb{N}$ tal que $\left|f_{n}\left(x_{k}-x\right)\right|<\frac{\varepsilon}{3}$, para todo $k \geq k_{n}$. Em particular, podemos escolher $k_{n}>n$ tal que $\left|f_{n}\left(x_{k_{n}}-x\right)\right|<\frac{\varepsilon}{3}$.

Ao fazer isso para cada $n \in \mathbb{N}$, obtemos subsequências $\left(x_{k_{n}}\right)_{n}$ de $\left(x_{n}\right)_{n}$ e $\left(f_{k_{n}}\right)_{n}$ de $\left(f_{n}\right)_{n}$. Dessa forma,

$$
\begin{aligned}
\left|f_{k_{n}}\left(x_{k_{n}}-x\right)\right| & =\left|f_{k_{n}}\left(x_{k_{n}}-x\right)-f_{n}\left(x_{k_{n}}-x\right)+f_{n}\left(x_{k_{n}}-x\right)\right| \\
& \leq\left|\left(f_{k_{n}}-f_{n}\right)\left(x_{k_{n}}-x\right)\right|+\left|f_{n}\left(x_{k_{n}}-x\right)\right| \\
& <\left|\left(f_{k_{n}}-f_{n}\right)\left(x_{k_{n}}\right)\right|+\left|\left(f_{k_{n}}-f_{n}\right)(x)\right|+\frac{\varepsilon}{3} .
\end{aligned}
$$

Como $\left(f_{n}\right)_{n}$ é $w$-Cauchy, segue que $f_{k_{n}}-f_{n} \stackrel{w}{\longrightarrow} 0$. Então, $\left(f_{k_{n}}-f_{n}\right)(x) \longrightarrow 0$, para todo $x \in X$.

De $x_{k_{n}} \stackrel{w}{\longrightarrow} x$, com $\left\|x_{k_{n}}\right\|=\|x\|=1$, e $f_{k_{n}}-f_{n} \stackrel{w}{\longrightarrow} 0$, e como $X$ tem a propriedade DP1, vem que $\left(f_{k_{n}}-f_{n}\right)\left(x_{k_{n}}\right) \longrightarrow 0$.

Assim, existe $n_{0} \in \mathbb{N}$ tal que, para todo $n \geq n_{0}$,

$$
\left|\left(f_{k_{n}}-f_{n}\right)(x)\right|<\frac{\varepsilon}{3} \quad \text { e } \quad\left|\left(f_{k_{n}}-f_{n}\right)\left(x_{k_{n}}\right)\right|<\frac{\varepsilon}{3} .
$$

Então, para todo $n \geq n_{0}$, temos que

$$
\left|f_{k_{n}}\left(x_{k_{n}}-x\right)\right|<\left|\left(f_{k_{n}}-f_{n}\right)\left(x_{k_{n}}\right)\right|+\left|\left(f_{k_{n}}-f_{n}\right)(x)\right|+\frac{\varepsilon}{3}<\frac{\varepsilon}{3}+\frac{\varepsilon}{3}+\frac{\varepsilon}{3}=\varepsilon,
$$

donde $f_{k_{n}}\left(x_{k_{n}}-x\right) \longrightarrow 0$.

Vejamos que $f_{n}\left(x_{n}-x\right) \longrightarrow 0$. Suponhamos, por absurdo, que $f_{n}\left(x_{n}-x\right) \nrightarrow 0$, isto é, existem $\theta>0$ e uma subsequência $\left(f_{n_{m}}\left(x_{n_{m}}-x\right)\right)_{m}$ tal que $\left|f_{n_{m}}\left(x_{n_{m}}-x\right)\right| \geq \theta$, para todo $m \in \mathbb{N}$.

Como $x_{n_{m}} \stackrel{w}{\longrightarrow} x$ em $S_{X}$ e $\left(f_{n_{m}}\right)_{m}$ é $w$-Cauchy em $X^{*}$, então, pelo que acabamos de mostrar, existem subsequências $\left(x_{n_{m_{i}}}\right)_{i}$ e $\left(f_{n_{m_{i}}}\right)_{i}$ tal que $f_{n_{m_{i}}}\left(x_{n_{m_{i}}}-x\right) \longrightarrow 0$, contradizendo o fato de que 
$\left|f_{n_{m}}\left(x_{n_{m}}-x\right)\right| \geq \theta$, para todo $m \in \mathbb{N}$. Logo, $f_{n}\left(x_{n}-x\right) \longrightarrow 0$.

(e) $\Rightarrow$ (a) Sejam $x_{n} \stackrel{w}{\longrightarrow} x$ em $X$, com $\left\|x_{n}\right\|=\|x\|=1$, e $f_{n} \stackrel{w}{\longrightarrow} f$ em $X^{*}$. Então, $\left(f_{n}\right)_{n}$ é $w$-Cauchy e, por hipótese, $f_{n}\left(x_{n}-x\right) \longrightarrow 0$. Como $f_{n} \stackrel{w}{\longrightarrow} f$, segue que $f_{n}(x) \longrightarrow f(x)$.

De modo análogo à demonstração de $(\mathbf{d}) \Rightarrow(\mathbf{a})$, segue que $f_{n}\left(x_{n}\right) \longrightarrow f(x)$. Logo, $X$ tem a propriedade DP1.

Em geral, as propriedades DP1 e KKP não são equivalentes (recíproca da Proposição 2.2.4 (b)). Com a hipótese adicional de o espaço ser reflexivo, obtemos tal recíproca.

Corolário 2.2.10. Seja X um espaço reflexivo. Se $X$ tem a propriedade DP1, então X tem a KKP.

Demonstração. Seja $x_{n} \stackrel{w}{\longrightarrow} x$ em $X$, com $\left\|x_{n}\right\|=\|x\|=1$. Como $X$ é reflexivo, pelo Teorema 2.2.9 (c), segue que o operador identidade $I d: X \rightarrow X$ é um operador DP1. Então, $I d\left(x_{n}\right) \longrightarrow I d(x)$, isto é, $x_{n} \longrightarrow x$. Logo, $\left\|x_{n}-x\right\| \longrightarrow 0$ e, portanto, $X$ tem a KKP.

Observação 2.2.11. Segue, da Proposição 2.2.4 (b) e do corolário anterior, que as propriedades KKP e DP1 são equivalentes em espaços de Banach reflexivos.

Exemplo 2.2.12. O espaço $L_{1}[0,1]$ não tem a propriedade KKP.

Seja $\left(r_{n}\right)_{n}$ a sequência formada pelas funções de Rademacher em $[0,1]$, isto é, para todo $n \in \mathbb{N}$ e $0 \leq t \leq 1$, temos

$$
r_{n}(t)=\operatorname{sgn}\left(\sin 2^{n} \pi t\right)
$$

Segue de [7, p. 139] que $\left\{r_{n}: n \in \mathbb{N}\right\}$ é ortonormal em $L_{2}[0,1]$.

Vejamos que $r_{n} \stackrel{w}{\longrightarrow} 0$ em $L_{2}[0,1]$. Como $\left\{r_{n}: n \in \mathbb{N}\right\}$ é ortonormal, segue pela desigualdade de Bessel (Teorema 1.3.2) que

$$
\sum_{n=1}^{\infty}\left|\left\langle\varphi, r_{n}\right\rangle\right|^{2} \leq\|\varphi\|^{2}
$$

para todo $\varphi \in L_{2}[0,1]$. Assim, $\sum_{n=1}^{\infty}\left|\left\langle\varphi, r_{n}\right\rangle\right|^{2}$ é convergente, então $\left\langle\varphi, r_{n}\right\rangle \longrightarrow 0$, para todo $\varphi \in$ $L_{2}[0,1]$. Logo, $r_{n} \stackrel{w}{\longrightarrow} 0$ em $L_{2}[0,1]$.

Como $L_{\infty}[0,1] \subseteq L_{2}[0,1]$ (vide [7, p. 28]), então $r_{n} \stackrel{w}{\longrightarrow} 0$ em $L_{1}[0,1]$.

Considere $f_{n}(t)=r_{n}(t)+1$, para todo $n \in \mathbb{N}$ e todo $0 \leq t \leq 1$. Como $r_{n} \stackrel{w}{\longrightarrow} 0$ em $L_{1}[0,1]$, então $f_{n} \stackrel{w}{\longrightarrow} 1$ em $L_{1}[0,1]$. Além disso, $\left\|f_{n}\right\|_{1}=1$, para todo $n \in \mathbb{N}$, pois

$$
\left\|f_{n}\right\|_{1}=\int_{0}^{1}\left|f_{n}(t)\right| d t=\int_{0}^{1}\left|r_{n}(t)+1\right| d t=1 .
$$

Entretando, $f_{n} \nrightarrow 1$ em norma, pois

$$
\left\|f_{n}-1\right\|_{1}=\left\|r_{n}\right\|_{1}=\int_{0}^{1}\left|r_{n}(t)\right| d t=1,
$$


para todo $n \in \mathbb{N}$

Logo, $L_{1}[0,1]$ não tem a propriedade KKP.

Observação 2.2.13. Como o espaço $L_{1}[0,1]$ tem a propriedade DP, então $L_{1}[0,1]$ também tem a propriedade DP1.

Assim, o espaço $L_{1}[0,1]$ tem a DP1 mas não tem a KKP.

O próximo exemplo evidenciará um espaço que não possui a propriedade DP1 e que nem todo espaço reflexivo possui a DP1 e a KKP.

Exemplo 2.2.14. O espaço de Banach $\ell_{2} \oplus_{\infty} \ell_{2}$ não tem a propriedade DP1. Considere $X=$ $\ell_{2} \oplus_{\infty} \ell_{2}$.

Sejam $y_{n} \stackrel{w}{\longrightarrow} y$ em $\ell_{2}$, com $\left\|y_{n}\right\|=\|y\|=1$, e $\left(e_{n}\right)_{n}$ a base canônica do $\ell_{2}$. Considere $x_{n}=$ $\left(e_{n}, y_{n}\right) \in X$ e $x=(0, y) \in X$.

Vejamos que $x_{n} \stackrel{w}{\longrightarrow} x$. Seja $\varphi \in\left(\ell_{2} \oplus_{\infty} \ell_{2}\right)^{*}$. Pela Proposição 1.1.26, existem $\varphi_{1}, \varphi_{2} \in \ell_{2}^{*}$ tais que $\varphi(a, b)=\varphi_{1}(a)+\varphi_{2}(b)$, para todo $a, b \in \ell_{2}$. Assim, $\varphi\left(e_{n}, y_{n}\right)=\varphi_{1}\left(e_{n}\right)+\varphi_{2}\left(y_{n}\right)$, para todo $n \in \mathbb{N}$.

Como $e_{n} \stackrel{w}{\longrightarrow} 0$ e $y_{n} \stackrel{w}{\longrightarrow} y$ em $\ell_{2}^{*}$, então $\varphi_{1}\left(e_{n}\right) \longrightarrow 0$ e $\varphi_{2}\left(y_{n}\right) \longrightarrow \varphi_{2}(y)$. Assim, $\varphi(x)=$ $\varphi\left(e_{n}, y_{n}\right) \longrightarrow(0, y)=x$.

Temos que $\left\|x_{n}\right\|=\|x\|=1$, pois

$$
\begin{gathered}
\left\|\left(e_{n}, y_{n}\right)\right\|=\sup \left\{\left\|e_{n}\right\|,\left\|y_{n}\right\|\right\}=1 \text { e } \\
\|(0, y)\|=\sup \{\|0\|,\|y\|\}=1 .
\end{gathered}
$$

Mas

$$
\begin{aligned}
\left\|x_{n}-x\right\| & =\left\|\left(e_{n}, y_{n}\right)-(0, y)\right\|=\left\|\left(e_{n}, y_{n}-y\right)\right\| \\
& =\sup \left\{\left\|e_{n}\right\|,\left\|y_{n}-y\right\|\right\} \geq 1,
\end{aligned}
$$

para todo $n \in \mathbb{N}$. Logo, $X$ não tem a KKP.

Pelo Teorema 1.1.23, $X$ é um espaço reflexivo. Logo, $X$ não tem a propriedade DP1.

Observação 2.2.15. Na Proposição 2.2.4 (c) e (d), vimos que as propriedades DP1 e KKP são preservadas por isomorfismos isométricos, ao contrário da propriedade DP, que é preservada por isomorfismos. No Exemplo 2.2.14, vimos que as propriedades DP1 e KKP não são preservadas apenas por isomorfismos.

O espaço $\ell_{2} \oplus_{2} \ell_{2}$ é um espaço de Hilbert (Proposição 1.3.3) e, portanto, tem a propriedade DP1 e a KKP. É fácil mostrar que os espaços $\ell_{2} \oplus_{\infty} \ell_{2}$ e $\ell_{2} \oplus_{2} \ell_{2}$ são isomorfos. Mas, como vimos no Exemplo 2.2.14, $\ell_{2} \oplus_{\infty} \ell_{2}$ não tem as propriedades DP1 e KKP.

Analogamente à propriedade DP, a propriedade DP1 é preservada em subespaços complementados. No caso da KKP, o subespaço precisa apenas ser fechado. Esses resultados serão apresentados nas próximas proposições.

Proposição 2.2.16. Seja $X$ um espaço de Banach com a propriedade DP1. Se $Z$ um subespaço complementado de $X$, então $Z$ tem a propriedade DP1. 
Demonstração. Seja $Z$ um subespaço complementado de $X$. Então, pela definição de subespaço complementado (Definição 1.1.7), existe uma projeção $P$ de $X$ sobre $Z$ e $Z$ é um subespaço fechado de $X$, portanto, um espaço de Banach.

Sejam $Y$ um espaço de Banach qualquer e $T: Z \rightarrow Y$ um operador $w$-compacto qualquer. Pela Proposição 1.1.51 (b), $T \circ P: X \rightarrow Y$ é $w$-compacto. Como $X$ tem a propriedade DP1, segue, pelo Teorema 2.2.9, que $T \circ P$ é um operador DP1.

Assim, dada uma sequência $z_{n} \stackrel{w}{\longrightarrow} z$ em $Z$, com $\left\|z_{n}\right\|=\|z\|=1$, temos que $(T \circ P)\left(z_{n}\right) \longrightarrow$ $(T \circ P)(z)$. Mas $(T \circ P)\left(z_{n}\right)=T\left(z_{n}\right)$ e $(T \circ P)(z)=T(z)$, donde $T\left(z_{n}\right) \longrightarrow T(z)$. Portanto, $T$ é um operador DP1.

Como $Y$ e $T$ eram quaisquer, segue que $Z$ tem a propriedade DP1.

Proposição 2.2.17. Seja $X$ um espaço de Banach com a KKP. Se $Z$ um subespaço fechado de $X$, então $Z$ tem a KKP.

Demonstração. Sejam $Z$ um subespaço fechado de $X$ e $z_{n} \stackrel{w}{\longrightarrow} z$ em $Z$ tais que $\left\|z_{n}\right\|=\|z\|=1$, para todo $n \in \mathbb{N}$.

Como $X$ tem a KKP, segue que $\left\|z_{n}-z\right\| \longrightarrow 0$ em $X$. Sendo $Z$ um subespaço fechado de $X$, então $\left\|z_{n}-z\right\| \longrightarrow 0$ em $Z$. Logo, $Z$ tem a KKP.

Nosso próximo objetivo é mostrar que as propriedades DP1 e a KKP são preservadas por somas diretas. A seguinte observação e o seguinte lema serão utilizados para esse fim.

Observação 2.2.18. Seja $I$ um conjunto qualquer e $\left\{a_{i}\right\}_{i \in I}$ não negativos. Então

$$
\sum_{i \in I} a_{i}=\sup \left\{\sum_{i \in J} a_{i}: J \subseteq I, J \text { finito }\right\} .
$$

Lema 2.2.19. Sejam $\left\{X_{i}: i \in I\right\}$ uma família de espaços de Banach e $X=\left(\oplus_{i} X_{i}\right)_{p}$, com $1 \leq p<\infty$. Considere $\left(x_{n}\right) \subset X, x \in X$, com $x=\left(x^{i}\right)$ e $x_{n}=\left(x_{n}^{i}\right)$, tais que $x_{n} \stackrel{w}{\longrightarrow} x$ e

$$
1=\left\|x_{n}\right\|^{p}=\left(\sum_{i}\left\|x_{n}^{i}\right\|^{p}\right)=\|x\|^{p}=\left(\sum_{i}\left\|x^{i}\right\|^{p}\right),
$$

para todo $n \in \mathbb{N}$. Então, $\left\|x_{n}^{i}\right\| \longrightarrow\left\|x^{i}\right\|, \forall i \in I$.

Demonstração. Seja $j \in I$. Suponha, por absurdo, que $\left\|x_{n}^{j}\right\| \nrightarrow\left\|x^{j}\right\|$.

Como limsup $\left\|x_{n}^{j}\right\| \geq\left\|x^{j}\right\|$, então existem $r>0$ e uma subsequência $\left(x_{n_{k}}^{j}\right)$ tais que $\left\|x_{n_{k}}^{j}\right\| \longrightarrow$ $r>\left\|x^{j}\right\|$. Assim:

$$
\begin{gathered}
1=\sum_{i}\left\|x^{i}\right\|^{p}=\sum_{i}\left\|x_{n_{k}}^{i}\right\|^{p}=\left\|x_{n_{k}}^{j}\right\|^{p}+\sum_{i \neq j}\left\|x_{n_{k}}^{i}\right\|^{p} \Longrightarrow \\
\sum_{i \neq j}\left\|x_{n_{k}}^{i}\right\|^{p}=1-\left\|x_{n_{k}}^{j}\right\|^{p}=\sum_{i \neq j}\left\|x^{i}\right\|^{p}+\left\|x^{j}\right\|^{p}-\left\|x_{n_{k}}^{j}\right\|^{p} \Longrightarrow
\end{gathered}
$$




$$
\sum_{i \neq j}\left\|x_{n_{k}}^{i}\right\|^{p} \longrightarrow \sum_{i \neq j}\left\|x^{i}\right\|^{p}+\left\|x^{j}\right\|^{p}-r^{p}<\sum_{i \neq j}\left\|x^{i}\right\|^{p}
$$

pois $\left\|x^{j}\right\|^{p}-r^{p}<0$

Para cada $n$, seja $\hat{x}=\left(\hat{x}_{n}^{i}\right) \in X$ definido por $\hat{x}_{n}^{i}=x_{n}^{i}, \forall i \neq j$, e $\hat{x}_{n}^{j}=0$. Analogamente, $\hat{x}=\left(\hat{x}^{i}\right)$.

Então, $\hat{x}_{n} \stackrel{w}{\longrightarrow} \hat{x}$ em $X$, pois $x_{n} \stackrel{w}{\longrightarrow} x$. Mas, por (2.1), temos que

$$
\lim _{k} \sum_{i \neq j}\left\|x_{n_{k}}^{i}\right\|^{p}<\lim _{k} \sum_{i \neq j}\left\|x^{i}\right\|^{p}
$$

donde $\lim _{k}\left\|\hat{x}_{n_{k}}\right\|<\|\hat{x}\|$. Absurdo, pois $\lim \sup \left\|\hat{x}_{n_{k}}\right\| \geq\|\hat{x}\|$. Logo, $\left\|x_{n}^{j}\right\| \longrightarrow\left\|x^{j}\right\|$.

Teorema 2.2.20. Sejam $\left\{X_{i}: i \in I\right\}$ uma familia de espaços de Banach e $X=\left(\oplus_{i} X_{i}\right)_{p}$, com $1 \leq p<\infty$. Então, $X$ tem a propriedade DP1 se, e somente se, para cada $i \in I, X_{i}$ tem a propriedade DP1.

Demonstração. $(\Rightarrow)$ Para cada $i \in I, X_{i}$ é um subespaço complementado em $X$. Assim, se $X$ tem a propriedade DP1, então, para cada $i \in I, X_{i}$ tem a propriedade DP1.

$(\Leftarrow)$ Sejam $\left(x_{n}\right) \subset X$ e $x \in X, \operatorname{com} x=\left(x^{i}\right)$ e $x_{n}=\left(x_{n}^{i}\right)$, tais que $x_{n} \stackrel{w}{\longrightarrow} x$ e, para todo $n \in \mathbb{N}$,

$$
1=\left\|x_{n}\right\|^{p}=\left(\sum_{i}\left\|x_{n}^{i}\right\|^{p}\right)=\|x\|^{p}=\left(\sum_{i}\left\|x^{i}\right\|^{p}\right) .
$$

Temos que $x_{n}^{i} \stackrel{w}{\longrightarrow} x^{i}$ em $X_{i}$, para todo $i \in I$.

Suponhamos que $X_{i}$ tem a propriedade DP1, $\forall i \in I$, mas $X$ não tem a propriedade DP1. Então, existe $\left(f_{n}\right) \subseteq X^{*}=\left(\oplus_{i} X_{i}^{*}\right)_{q}$, com $\frac{1}{p}+\frac{1}{q}=1$ e $f_{n}=\left(f_{n}^{i}\right)$, tal que $f_{n} \stackrel{w}{\longrightarrow} 0$ e $\left|f_{n}\left(x_{n}\right)\right| \nrightarrow 0$. Do Teorema 1.1.26, segue que

$$
\left|f_{n}\left(x_{n}\right)\right|=\left|\sum_{i} f_{n}^{i}\left(x_{n}^{i}\right)\right| \nrightarrow 0 .
$$

Como $f_{n} \stackrel{w}{\longrightarrow} 0$, então $\left(f_{n}(x)\right)_{n}$ é uma sequência limitada. Assim, pelo Teorema de BanachSteinhaus (1.1.1), $\left\|f_{n}\right\|<\infty$, para todo $n \in \mathbb{N}$. Podemos supor, sem perda de generalidade, que $\left\|f_{n}\right\| \leq 1$.

Para todo $n \in \mathbb{N}$,

$$
\left|\sum_{i} f_{n}^{i}\left(x_{n}^{i}\right)\right| \leq\left\|f_{n}\right\|\left\|x_{n}\right\| \leq 1 .
$$

Mas toda sequência limitada em $\mathbb{K}$ admite uma subsequência convergente. Então, existem uma sequência $\left(n_{k}\right) \subset \mathbb{N}$ e $0<s \leq 1$ tais que $\left|\sum_{i} f_{n_{k}}^{i}\left(x_{n_{k}}^{i}\right)\right| \longrightarrow s$.

Seja $0<\varepsilon<\frac{s^{p}}{2^{p+1}}$. Temos que $2(2 \varepsilon)^{\frac{1}{p}}<s$ e, como $2 \varepsilon<1$, vem que $2 \varepsilon \leq(2 \varepsilon)^{\frac{1}{p}}$ e $2 \varepsilon+(2 \varepsilon)^{\frac{1}{p}}<s$.

Tomemos um conjunto $I_{0} \subseteq I$, finito, tal que

$$
\sum_{i \notin I_{0}}\left\|x^{i}\right\|^{p}<\varepsilon
$$


e tomemos $N>0$ tal que, para todo $k \geq N$, temos

$$
\begin{gathered}
|| \sum_{i} f_{n_{k}}^{i}\left(x_{n_{k}}^{i}\right)|-s|<\varepsilon, \\
\sum_{i \in I_{0}}\left|f_{n_{k}}^{i}\left(x_{n_{k}}^{i}\right)\right|<\varepsilon \quad \mathrm{e} \\
\sum_{i \in I_{0}}\left|\left\|x_{n_{k}}^{i}\right\|^{p}-\left\|x^{i}\right\|^{p}\right|<\varepsilon .
\end{gathered}
$$

Isso é possível, pois, pelo Lema 2.2.19, temos que $\left\|x_{n_{k}}^{i}\right\| \longrightarrow\left\|x^{i}\right\|$ e $X_{i}$ tem a propriedade DP1, $\forall i \in I$.

$$
\begin{aligned}
& \text { Como } \sum_{i}\left\|x_{n}^{i}\right\|^{p}=\sum_{i}\left\|x^{i}\right\|^{p}=1 \text {, temos que } \\
& \qquad \begin{aligned}
\sum_{i \notin I_{0}}\left\|x_{n_{k}}^{i}\right\|^{p} & =1-\sum_{i \in I_{0}}\left\|x_{n_{k}}^{i}\right\|^{p} \\
& =\sum_{i \in I_{0}}\left\|x^{i}\right\|^{p}+\sum_{i \notin I_{0}}\left\|x^{i}\right\|^{p}-\sum_{i \in I_{0}}\left\|x_{n_{k}}^{i}\right\|^{p} \\
& =\sum_{i \in I_{0}}\left(\left\|x^{i}\right\|^{p}-\left\|x_{n_{k}}^{i}\right\|^{p}\right)+\sum_{i \notin I_{0}}\left\|x^{i}\right\|^{p} \\
& \leq \sum_{i \in I_{0}}\left|\left\|x^{i}\right\|^{p}-\left\|x_{n_{k}}^{i}\right\|^{p}\right|+\sum_{i \notin I_{0}}\left\|x^{i}\right\|^{p} .
\end{aligned}
\end{aligned}
$$

Por $(2.2)$ e $(2.5)$, temos que

$$
\sum_{i \notin I_{0}}\left\|x_{n_{k}}^{i}\right\|^{p}<2 \varepsilon
$$

Por (2.3), (2.4) e (2.6), segue que

$$
\begin{aligned}
s-\varepsilon & <\left|\sum_{i} f_{n_{k}}^{i}\left(x_{n_{k}}^{i}\right)\right|<\left|\sum_{i \in I_{0}} f_{n_{k}}^{i}\left(x_{n_{k}}^{i}\right)\right|+\left|\sum_{i \notin I_{0}} f_{n_{k}}^{i}\left(x_{n_{k}}^{i}\right)\right| \\
& <\varepsilon+\left|\sum_{i \notin I_{0}} f_{n_{k}}^{i}\left(x_{n_{k}}^{i}\right)\right| \leq \varepsilon+\left(\sum_{i \notin I_{0}}\left\|x_{n_{k}}^{i}\right\|^{p}\right)^{\frac{1}{p}}\left\|f_{n_{k}}\right\|<\varepsilon+(2 \varepsilon)^{\frac{1}{p}} .
\end{aligned}
$$

Assim, $s-\varepsilon<\varepsilon+(2 \varepsilon)^{\frac{1}{p}}$, donde $s<2 \varepsilon+(2 \varepsilon)^{\frac{1}{p}}$. Absurdo!

Logo, $f_{n}\left(x_{n}\right) \longrightarrow 0$ e, portanto, $X$ tem a propriedade DP1.

Observação 2.2.21. Dos Exemplos 2.1 .4 e 2.1.13, temos que $\ell_{p}$, para $1<p<\infty$, não possui a propriedade DP e $\ell_{\infty}$ possui a propriedade DP. Pelo Teorema 2.2.20, segue que $\ell_{p}$, para $1 \leq p<\infty$, possui a propriedade DP1. Assim, $\ell_{p}$, para $1 \leq p \leq \infty$, possui a propriedade DP1 e $\ell_{p}$, para $1<p<\infty$, não possui a propriedade DP.

Teorema 2.2.22. Sejam $\left\{X_{i}: i \in I\right\}$ uma família de espaços de Banach e $X=\left(\oplus_{i} X_{i}\right)_{p}$, com $1 \leq p<\infty$. Então $X$ tem a KKP se, e somente se, para cada $i \in I, X_{i}$ tem a KKP. 
Demonstração. $(\Rightarrow)$ Para cada $i \in I, X_{i}$ é um subespaço complementado em $X$. Então $X_{i}$ é fechado em $X$. Assim, se $X$ tem a KKP, então, para cada $i \in I, X_{i}$ tem a KKP.

$(\Leftarrow)$ Suponhamos que $X_{i}$ tem a KKP, $\forall i \in I$, mas $X$ não tem a KKP. Então, existem $\left(x_{n}\right) \subset X$, $x \in X, \operatorname{com} x=\left(x^{i}\right)$ e $x_{n}=\left(x_{n}^{i}\right)$, tais que $x_{n} \stackrel{w}{\longrightarrow} x$, para todo $n \in \mathbb{N}$,

$$
1=\left\|x_{n}\right\|^{p}=\left(\sum_{i}\left\|x_{n}^{i}\right\|^{p}\right)=\|x\|^{p}=\left(\sum_{i}\left\|x^{i}\right\|^{p}\right)
$$

e $\left\|x_{n}-x\right\| \nrightarrow 0$.

Para todo $i \in I$, temos que $x_{n}^{i} \stackrel{w}{\longrightarrow} x^{i}$ em $X_{i}$ e, pelo Lema 2.2.19, $\left\|x_{n}^{j}\right\| \longrightarrow\left\|x^{j}\right\|$. Como $X_{i}$ tem a KKP, segue que $\left\|x_{n}^{j}-x^{j}\right\| \longrightarrow 0$, para todo $j \in I$.

Mas $\left\|x_{n}\right\|=1$ e $\|x\|=1$, donde $\left\|x_{n}\right\|+\|x\|=2$. Assim, $\left\|x_{n}-x\right\| \leq\left\|x_{n}\right\|+\|x\|=2$, ou seja, $\left\|x_{n}-x\right\|^{p} \leq 2^{p}$.

Dessa maneira, existem uma subsequência $\left(x_{n_{k}}\right)_{k}$ e $0<s \leq 2^{p}$ tais que

$$
\left\|x_{n_{k}}-x\right\|^{p}=\sum_{i}\left\|x_{n_{k}}^{i}-x^{i}\right\|^{p} \longrightarrow s
$$

Sejam $0<\varepsilon<\frac{s}{2+2^{p}+2^{p+1}}$ e $I_{0} \subset I$, finito, tais que

$$
\sum_{i \notin I_{0}}\left\|x^{i}\right\|^{p}<\varepsilon
$$

e tomemos $N>0$ tal que, para todo $k \geq N$, temos

$$
\begin{gathered}
\left|\sum_{i}\left\|x_{n_{k}}^{i}-x^{i}\right\|^{p}-s\right|<\varepsilon, \\
\sum_{i \in I_{0}}\left\|x_{n_{k}}^{i}-x^{i}\right\|^{p}<\varepsilon \quad \mathrm{e} \\
\sum_{i \in I_{0}}\left|\left\|x_{n_{k}}^{i}\right\|^{p}-\left\|x^{i}\right\|^{p}\right|<\varepsilon .
\end{gathered}
$$

Como $\sum_{i}\left\|x_{n}^{i}\right\|^{p}=\sum_{i}\left\|x^{i}\right\|^{p}=1$, segue que

$$
\begin{aligned}
\sum_{i \notin I_{0}}\left\|x_{n_{k}}^{i}\right\|^{p} & =1-\sum_{i \in I_{0}}\left\|x_{n_{k}}^{i}\right\|^{p} \\
& =\sum_{i \in I_{0}}\left\|x^{i}\right\|^{p}+\sum_{i \notin I_{0}}\left\|x^{i}\right\|^{p}-\sum_{i \in I_{0}}\left\|x_{n_{k}}^{i}\right\|^{p} \\
& =\sum_{i \in I_{0}}\left(\left\|x^{i}\right\|^{p}-\left\|x_{n_{k}}^{i}\right\|^{p}\right)+\sum_{i \notin I_{0}}\left\|x^{i}\right\|^{p} \\
& \leq \sum_{i \in I_{0}}\left|\left\|x^{i}\right\|^{p}-\left\|x_{n_{k}}^{i}\right\|^{p}\right|+\sum_{i \notin I_{0}}\left\|x^{i}\right\|^{p} .
\end{aligned}
$$

Por (2.7) e (2.10), vem que

$$
\sum_{i \notin I_{0}}\left\|x_{n_{k}}^{i}\right\|^{p}<2 \varepsilon
$$


Por (2.7), (2.8), (2.9) e (2.6), temos que

$$
\begin{aligned}
s-\varepsilon & <\sum_{i}\left\|x_{n_{k}}^{i}-x^{i}\right\|^{p}=\sum_{i \in I_{0}}\left\|x_{n_{k}}^{i}-x^{i}\right\|^{p}+\sum_{i \notin I_{0}}\left\|x_{n_{k}}^{i}-x^{i}\right\|^{p} \\
& <\varepsilon+\sum_{i \notin I_{0}}\left\|x_{n_{k}}^{i}-x^{i}\right\|^{p} \leq \varepsilon+\sum_{i \notin I_{0}}\left(\left\|x_{n_{k}}^{i}\right\|+\left\|x^{i}\right\|\right)^{p} \\
& \leq \varepsilon+\sum_{i \notin I_{0}}\left(2 \max \left\{\left\|x_{n_{k}}^{i}\right\|,\left\|x^{i}\right\|\right\}\right)^{p}=\varepsilon+2^{p} \sum_{i \notin I_{0}}\left(\max \left\{\left\|x_{n_{k}}^{i}\right\|,\left\|x^{i}\right\|\right\}\right)^{p} \\
& \leq \varepsilon+2^{p} \sum_{i \notin I_{0}}\left(\left\|x_{n_{k}}^{i}\right\|^{p}+\left\|x^{i}\right\|^{p}\right)<\varepsilon+2^{p}(2 \varepsilon+\varepsilon)=\varepsilon\left(1+2^{p}+2^{p+1}\right) .
\end{aligned}
$$

Então, $s<\varepsilon\left(2+2^{p}+2^{p+1}\right)$. Absurdo! Logo, $\left\|x_{n}-x\right\| \longrightarrow 0$ e, portanto, $X$ tem a KKP.

Vimos que se $X$ é um espaço de Banach tal que $X^{*}$ tem a propriedade DP, então $X$ também tem a propriedade DP. Mas isto não é fálido para a propriedade DP1. Veremos no Proposição 4.4.7 que o espaço $K(H)$, com $H$ um espaço de Hilbert de dimensão infinita, não tem a propriedade DP1, mas o seu dual $\mathscr{L}_{1}(H)$ tem a propriedade DP1 (Teorema 4.1.30).

No Exemplo 2.1.10, foi visto que o espaço dado por Stegall tem a propriedade DP, mas o seu dual não tem a propriedade DP. Esse espaço se comporta da mesma maneira com relação à propriedade DP1, isto é, ele tem a propriedade DP1, mas o seu dual, não.

Para estudarmos a demonstração de que o dual desse espaço não tem a propriedade DP1, serão necessários dois resultados: uma extensão da desigualdade de Minkowski (vide [5, p. 21]) e um teorema conhecido como The Principle of Local Selection.

Extensão da Desigualdade de Minkowski: Se $x_{i j} \in \mathbb{K}$, para $i=1,2, \ldots, n$ e $j=1,2, \ldots, m$ e $p>1$, então

$$
\left(\sum_{i=1}^{n}\left(\sum_{j=1}^{m}\left|x_{i j}\right|\right)^{p}\right)^{\frac{1}{p}} \leq \sum_{j=1}^{m}\left(\sum_{i=1}^{n}\left|x_{i j}\right|^{p}\right)^{\frac{1}{p}}
$$

Teorema 2.2.23 (The Principle of Local Selection). Sejam $T \in L(X, Y),\left(F_{\lambda}\right)_{\lambda \in \Lambda}$ uma rede de subespaços de $Y$, ordenados pela inclusão, com $Y_{0}=\bigcup F_{\lambda}$ denso em $Y$. Para cada $\lambda$, existe $S_{\lambda}: F_{\lambda} \longrightarrow X$ tal que $T \circ S_{\lambda}=I d_{F_{\lambda}}$ e limsup $\left\|S_{\lambda}\right\| \leq \alpha<\infty$. Então, $T$ é um operador quociente de $X$ sobre $Y$ e $T^{*}\left(Y^{*}\right)$ é complementado em $X^{*}$ por uma projeção de norma menor ou igual a $\alpha\|T\|$.

(vide [16, p. 23])

Exemplo 2.2.24. Para cada $n \in \mathbb{N}$, denotaremos por $\ell_{2}^{(n)}$ o espaço vetorial do $\mathbb{K}^{n}$ com a norma de $\ell_{2}$. Seja $X=\left(\oplus_{n=1}^{\infty} \ell_{2}^{(n)}\right)_{1}$, isto é, cada $x \in X$ é tal que $x=\left(x^{(n)}\right)_{n}$, em que $x^{(n)} \in \ell_{2}^{(n)}$ e $\|x\|=\sum_{n=1}^{\infty}\left\|x^{(n)}\right\|_{2}=\sum_{n=1}^{\infty}\left(\sum_{i=1}^{n}\left|x_{i}^{(n)}\right|^{2}\right)^{\frac{1}{2}}$. Temos que $X^{*}=\left(\oplus_{n=1}^{\infty} \ell_{2}^{(n)}\right)_{\infty}$.

No Exemplo 2.1.10, vimos que se $X$ tem a propriedade DP, então $X$ tem a propriedade DP1. Mostraremos que $X^{*}$ não tem a propriedade DP1. 
Observemos que $X^{*}$ é isometricamente isomorfo a $\left(\oplus_{n=1}^{\infty} \ell_{2}^{(2 n)}\right)_{\infty} \oplus_{\infty}\left(\oplus_{n=1}^{\infty} \ell_{2}^{(2 n-1)}\right)_{\infty}$. Vamos mostrar que para qualquer sequência crescente $\left(n_{k}\right) \subseteq \mathbb{N}$, temos que $\left(\oplus_{k=1}^{\infty} \ell_{2}^{\left(n_{k}\right)}\right)_{\infty}$ contém uma cópia complementada isometricamente isomorfa a $\ell_{2}$. Denotaremos por $Z=\left(\oplus_{k=1}^{\infty} \ell_{2}^{\left(n_{k}\right)}\right)_{1}$ e $Z^{*}=\left(\oplus_{k=1}^{\infty} \ell_{2}^{\left(n_{k}\right)}\right)_{\infty}$

Consideremos $T:\left(\oplus_{k=1}^{\infty} \ell_{2}^{\left(n_{k}\right)}\right)_{1} \longrightarrow \ell_{2}$ dado por

$$
T(x)=\left(x_{1}^{\left(n_{1}\right)}+x_{1}^{\left(n_{2}\right)}+\cdots, x_{2}^{\left(n_{1}\right)}+x_{2}^{\left(n_{2}\right)}+\cdots, \ldots, x_{k}^{\left(n_{k}\right)}+x_{k}^{\left(n_{k+1}\right)}+\cdots, \ldots\right),
$$

em que $x=\left(\left(x_{1}^{\left(n_{1}\right)}, x_{2}^{\left(n_{1}\right)}, \ldots, x_{n_{1}}^{\left(n_{1}\right)}\right),\left(x_{1}^{\left(n_{2}\right)}, x_{2}^{\left(n_{2}\right)}, \ldots, x_{n_{2}}^{\left(n_{2}\right)}\right), \ldots,\left(x_{1}^{\left(n_{k}\right)}, x_{2}^{\left(n_{k}\right)}, \ldots, x_{n_{k}}^{\left(n_{k}\right)}\right), \ldots\right) \in\left(\oplus_{k=1}^{\infty} \ell_{2}^{\left(n_{k}\right)}\right)_{1}$.

Vejamos que $T$ está bem definido, isto é, $T(x) \in \ell_{2}$, para todo $x \in\left(\oplus_{k=1}^{\infty} \ell_{2}^{\left(n_{k}\right)}\right)_{1}$. Seja $x \in$ $\left(\oplus_{k=1}^{\infty} \ell_{2}^{\left(n_{k}\right)}\right)_{1}$. Temos que $\sum_{k=1}^{\infty}\left(\sum_{i=1}^{n_{k}}\left|x_{i}^{\left(n_{k}\right)}\right|^{2}\right)^{\frac{1}{2}}<\infty$. Pela extensão da desigualdade de Minkowski, vem que

$$
\left(\sum_{i=1}^{\infty}\left|\sum_{k=1}^{\infty} x_{i}^{\left(n_{k}\right)}\right|^{2}\right)^{\frac{1}{2}} \leq \sum_{k=1}^{\infty}\left(\sum_{i=1}^{n_{k}}\left|x_{i}^{\left(n_{k}\right)}\right|^{2}\right)^{\frac{1}{2}}<\infty
$$

(Notemos que poderíamos escrever $\sum_{k=1}^{\infty}\left(\sum_{i=1}^{\infty}\left|x_{i}^{\left(n_{k}\right)}\right|^{2}\right)^{\frac{1}{2}}$ se considerássemos $x_{i}^{\left(n_{k}\right)}=0$, para $i>k$.)

Verifiquemos que $T$ é contínuo. Considerando a mesma desigualdade, para $x \in X$, temos que

$$
\|T(x)\|_{2}=\left(\sum_{i=1}^{\infty}\left|\sum_{k=1}^{\infty} x_{i}^{\left(n_{k}\right)}\right|^{2}\right)^{\frac{1}{2}} \leq \sum_{k=1}^{\infty}\left(\sum_{i=1}^{n_{k}}\left|x_{i}^{\left(n_{k}\right)}\right|^{2}\right)^{\frac{1}{2}}=\|x\|
$$

Além disso, $\left\|T\left(e_{1}\right)\right\|=\left\|e_{1}\right\|=1$. Logo, $\|T\|=1$.

Verifiquemos também que $T^{*}\left(\ell_{2}\right)$ é um subespaço complementado em $Z^{*}$. Para tanto usaremos The Principle of Local Selection (Teorema 2.2.23).

Seja $Y_{i}$ o espaço vetorial gerado por $e_{1}, \cdots, e_{i}$, para cada $i \in \mathbb{N}$, em que $e_{i}$ é o $i$-ésimo vetor da base canônica de $\ell_{2}$. Então

$$
Y_{1} \subset Y_{2} \subset \cdots \subset Y_{n} \subset \cdots \text { e } \bigcup_{n=1}^{\infty} Y_{n}=\ell_{2} .
$$

Consideremos o operador $S_{i}: Y_{i} \rightarrow X$, para cada $i \in \mathbb{N}$, definido por

$$
S_{i}\left(\alpha_{1} e_{1}+\cdots+\alpha_{i} e_{i}\right)=(0,(0,0), \cdots, \overbrace{\left(\alpha_{1}, \cdots, \alpha_{i}\right)}^{i \text {-ésimo }},(0, \cdots, 0), \cdots),
$$


para todo $\left(\alpha_{1} e_{1}+\cdots+\alpha_{i} e_{i}\right) \in Y_{i}$. Assim, temos que

$$
\begin{aligned}
T \circ S_{i}\left(\alpha_{1} e_{1}+\cdots+\alpha_{i} e_{i}\right) & =T\left(0,(0,0), \cdots,\left(\alpha_{1}, \cdots, \alpha_{i}\right),(0, \cdots, 0), \cdots\right) \\
& =\left(\alpha_{1}, \cdots, \alpha_{i}, 0, \cdots\right)=\alpha_{1} e_{1}+\cdots+\alpha_{i} e_{i},
\end{aligned}
$$

para todo $\left(\alpha_{1} e_{1}+\cdots+\alpha_{i} e_{i}\right) \in Y_{i}$ e para cada $i \in \mathbb{N}$. Portanto, $T \circ S_{i}=I d_{Y_{i}}$, em que $I d_{Y_{i}}$ é o operador identidade em $Y_{i}$.

Além disso,

$$
\begin{aligned}
\left\|S_{i}\right\| & =\sup \left\{\left\|S_{i}\left(\alpha_{1} e_{1}+\cdots+\alpha_{i} e_{i}\right)\right\|:\left\|\left(\alpha_{1} e_{1}+\cdots+\alpha_{i} e_{i}\right)\right\| \leq 1\right\} \\
& =\sup \left\{\left\|S_{i}\left(0,(0,0), \cdots,\left(\alpha_{1}, \cdots, \alpha_{i}\right),(0, \cdots, 0), \cdots\right)\right\|:\left\|\left(\alpha_{1} e_{1}+\cdots+\alpha_{i} e_{i}\right)\right\| \leq 1\right\},
\end{aligned}
$$

para todo $i \in \mathbb{N}$. Assim, $\lim _{i \rightarrow \infty}\left\|S_{i}\right\|=1<\infty$.

Logo, pelo Teorema 2.2 .23 , $T$ é sobrejetor e $T^{*}\left(\ell_{2}\right)$ é um subespaço complementado em $Z^{*}$.

Resta-nos provar que $T^{*}: \ell_{2}^{*} \rightarrow Z^{*}$ é uma isometria, isto é, $\left\|T^{*}(\varphi)\right\|=\|\varphi\|$, para todo $\varphi \in$ $\ell_{2}^{*} \cong \ell_{2}$. Primeiramente, vejamos que $T\left(B_{Z}\right)$ é denso em $B_{\ell_{2}}$. Observe que $T\left(e_{j}^{\left(n_{k}\right)}\right)=e_{j}$, para todo $k \in \mathbb{N}$ e todo $j \in \mathbb{N}$.

Seja $y=\sum_{j=1}^{\infty} \gamma_{j} e_{j} \in B_{\ell_{2}}$. Temos que $\sum_{j=1}^{k} \gamma_{j} e_{j} \longrightarrow y \mathrm{e}$

$$
\sum_{j=1}^{k} \gamma_{j} e_{j}=\sum_{j=1}^{k} \gamma_{j} T\left(e_{j}^{\left(n_{k}\right)}\right)=T\left(\sum_{j=1}^{k} \gamma_{j} e_{j}^{\left(n_{k}\right)}\right) .
$$

Mas

$$
\begin{aligned}
\left\|\sum_{j=1}^{k} \gamma_{j} e_{j}^{\left(n_{k}\right)}\right\| & =\left\|\left(0,(0,0),(0,0,0), \ldots,\left(\gamma_{1}, \ldots, \gamma_{k}\right),(0, \ldots, 0), \ldots\right)\right\| \\
& =\left\|\left(\gamma_{1}, \ldots, \gamma_{k}\right)\right\|_{2}=\left(\sum_{j=1}^{k}\left|\gamma_{j}\right|^{2}\right)^{\frac{1}{2}} \leq\left(\sum_{j=1}^{\infty}\left|\gamma_{j}\right|^{2}\right)^{\frac{1}{2}}=\|y\| \leq 1 .
\end{aligned}
$$

Então $\sum_{j=1}^{k} \gamma_{j} e_{j}^{\left(n_{k}\right)} \in B_{Z}$. Logo, $y \in \overline{T\left(B_{Z}\right)}$ e, portanto, $B_{\ell_{2}} \subseteq \overline{T\left(B_{Z}\right)}$.

Seja $x \in B_{Z}$. Temos que $\|T(x)\| \leq\|T\|\|x\| \leq 1$, donde $T(x) \in B_{\ell_{2}}$. Assim, $T\left(B_{Z}\right) \subseteq B_{\ell_{2}}$. Mas, $\overline{T\left(B_{Z}\right)} \subseteq \overline{B_{\ell_{2}}}=B_{\ell_{2}}$. Logo, $\overline{T\left(B_{Z}\right)}=B_{\ell_{2}}$, isto é, $T\left(B_{Z}\right)$ é denso em $B_{\ell_{2}}$.

Seja $\varphi \in \ell_{2}^{*}$. Temos

$$
\begin{gathered}
\|\varphi\|=\sup _{y \in T\left(B_{Z}\right)}|\varphi(y)| \\
\left\|T^{*}(\varphi)\right\|=\sup _{x \in B_{Z}}\left|T^{*}(\varphi)(x)\right|=\sup _{x \in B_{Z}}|\varphi(T(x))|=\sup _{y \in T\left(B_{Z}\right)}|\varphi(y)|=\|\varphi\|
\end{gathered}
$$

Logo, $T^{*}$ é uma isometria e $T^{*}$ é injetor.

Como $T^{*}$ é linear, contínuo e injetor, segue, pelo Teorema da Aplicação Aberta (Teorema 
1.1.2), que $\left(T^{*}\right)^{-1}: T^{*}\left(\ell_{2}\right) \longrightarrow \ell_{2}$ é contínuo. Consequentemente, $T^{*}\left(\ell_{2}\right)$ é isomorfo a $\ell_{2}$, isto é, $\left(\oplus_{k=1}^{\infty} \ell_{2}^{\left(n_{k}\right)}\right)_{\infty}$ contém uma cópia complementada isometricamente isomorfa a $\ell_{2}$.

Portanto, $X^{*}$ contém uma cópia complementada isometricamente isomorfa a $\ell_{2} \oplus_{\infty} \ell_{2}$. Mas, pelo Exemplo 2.2.14, $\ell_{2} \oplus_{\infty} \ell_{2}$ não tem a propriedade DP1, então $X^{*}$ não tem a propriedade DP1.

O teorema abaixo nos mostra que as propriedades DP1 e KKP são preservadas por espaço quociente com algumas hipóteses sobre o espaço.

Teorema 2.2.25. Sejam $X$ um espaço de Banach w-sequencialmente completo e $M \subseteq X$ um subespaço reflexivo. Então

(a) Se $X$ tem a propriedade DP1, então $X / M$ tem a propriedade DP1;

(b) Se $X$ tem a KKP, então $X / M$ tem a KKP.

Demonstração. (a) Suponhamos, por absurdo, que $X / M$ não tem a propriedade DP1. Então, existem $y_{n} \stackrel{w}{\longrightarrow} y \mathrm{em} X / M$, com $\left\|y_{n}\right\|=\|y\|=1$, e $f_{n} \stackrel{w}{\longrightarrow} 0$ em $(X / M)^{*}$ tais que $f_{n}\left(y_{n}\right) \nrightarrow 0$, isto é, existem $\theta>0$ e uma subsequência $\left(f_{n_{i}}\left(x_{n_{i}}\right)\right)_{i}$ tais que $\left|f_{n_{i}}\left(x_{n_{i}}\right)\right| \geq \theta$, para todo $i \in \mathbb{N}$.

Como $M$ é reflexivo, pelo Teorema 1.1.40, existem uma subsequência $\left(y_{n_{i_{k}}}\right)_{k}$ e uma sequência $\left(x_{k}\right)_{k} w$-Cauchy em $X$ tais que $\pi\left(x_{k}\right)=y_{n_{i_{k}}}$, para todo $k \in \mathbb{N}$, em que $\pi: X \longrightarrow X / M$ é a aplicação quociente.

Como $X$ é $w$-sequencialmente completo, pela Definição 1.1.38, existe $x \in X$ tal que $x_{k} \stackrel{w}{\longrightarrow} x$ em $X$. Assim, $y_{n_{i_{k}}}=\pi\left(x_{k}\right) \stackrel{w}{\longrightarrow} \pi(x)$, donde $\pi(x)=y$ e $\left\|\pi\left(x_{k}\right)\right\|=\|\pi(x)\|=1$.

Para todo $k \in \mathbb{N}$, sabemos que

$$
1=\left\|y_{n_{i_{k}}}\right\|=\inf _{m \in M}\left\|x_{k}-m\right\| .
$$

Tomemos $m_{k} \in M$ tal que $1 \leq\left\|x_{k}-m_{k}\right\|<1+2^{-k}$, para todo $k \in \mathbb{N}$. Então, $\left\|x_{k}-m_{k}\right\| \longrightarrow 1$ e, como $\left(x_{k}\right)_{k}$ é limitado, temos que

$$
\left\|m_{k}\right\| \leq\left\|m_{k}-x_{k}\right\|+\left\|x_{k}\right\|<1+2^{-k}+\left\|x_{k}\right\| .
$$

Logo, $\left(m_{k}\right)_{k}$ é limitado.

Como $X$ é reflexivo, pelo Corolário 1.1.37, existe uma subsequência $\left(m_{k_{p}}\right)_{p}$ tal que $m_{k_{p}} \stackrel{w}{\longrightarrow} m$ em $M$. Podemos supor, sem perda de generalidade, que $m_{k} \stackrel{w}{\longrightarrow} m$ em $M$. Consequentemente, $x_{k}-m_{k} \stackrel{w}{\longrightarrow} x-m$ em $M$ e $\left\|x_{k}-m_{k}\right\| \longrightarrow 1$. Pela Proposição 1.1.34, vem que $\|x-m\| \leq$ $\liminf _{k}\left\|x_{k}-m_{k}\right\|=1$.

Por outro lado,

$$
1=\|\pi(x)\|=\|\pi(x-m)\| \leq\|\pi\|\|x-m\|=\|x-m\| .
$$

Assim, $x_{k}-m_{k} \stackrel{w}{\longrightarrow} x-m$ em $X$ e $\left\|x_{k}-m_{k}\right\| \longrightarrow\|x-m\|=1$.

Em vista da definição de operador adjunto (Definição 1.1.11), temos que $\pi^{*}:(X / M)^{*} \longrightarrow X^{*}$ e $\pi^{*}\left(f_{n_{i_{k}}}\right)=f_{n_{i_{k}}} \circ \pi$. Como $f_{n} \stackrel{w}{\longrightarrow} 0$ em $(X / M)^{*}$, temos que $\pi^{*}\left(f_{n_{i_{k}}}\right) \stackrel{w}{\longrightarrow} 0$ em $X^{*}$. 
Como $X$ tem a propriedade DP1, segue que $\pi^{*}\left(f_{n_{i_{k}}}\right)\left(x_{k}-m_{k}\right) \longrightarrow 0$. Dessa forma,

$$
f_{n_{i_{k}}}\left(y_{n_{i_{k}}}\right)=f_{n_{i_{k}}}\left(\pi\left(x_{k}\right)\right)=f_{n_{i_{k}}}\left(\pi\left(x_{k}-m_{k}\right)\right)=\left(\pi^{*} f_{n_{i_{k}}}\right)\left(x_{k}-m_{k}\right) \longrightarrow 0,
$$

contradizendo o fato de que $\left|f_{n_{i}}\left(x_{n_{i}}\right)\right| \geq \theta$, para todo $i \in \mathbb{N}$.

Logo, $X / M$ tem a propriedade DP1.

(b) Suponhamos, por absurdo, que $X / M$ não tem a KKP. Então, existe $y_{n} \stackrel{w}{\longrightarrow} y \mathrm{em} X / M$ com $\left\|y_{n}\right\|=\|y\|=1$ tal que $\left\|y_{n}-y\right\| \nrightarrow 0$, isto é, existem $\theta>0$ e uma subsequência $\left(\left\|y_{n_{i}}-y\right\|\right)_{i}$ tais que $\left\|y_{n_{i}}-y\right\| \geq \theta$, para todo $i \in \mathbb{N}$.

Pelo Teorema 1.1.40, existem uma subsequência $\left(y_{n_{i_{k}}}\right)_{k}$ e uma sequência $\left(x_{k}\right)_{k} w$-Cauchy em $X$ tais que $\pi\left(x_{k}\right)=y_{n_{i_{k}}}$, para todo $k \in \mathbb{N}$, em que $\pi: X \longrightarrow X / M$ é a aplicação quociente.

Como $X$ é $w$-sequencialmente completo, pela Definição 1.1.38, existe $x \in X$ tal que $x_{k} \stackrel{w}{\longrightarrow} x$ em $X$. Assim, $y_{n_{i_{k}}}=\pi\left(x_{k}\right) \stackrel{w}{\longrightarrow} \pi(x)$, donde $\pi(x)=y$ e $\left\|\pi\left(x_{k}\right)\right\|=\|\pi(x)\|=1$.

Para todo $k \in \mathbb{N}$, sabemos que

$$
1=\left\|y_{n_{i_{k}}}\right\|=\inf _{m \in M}\left\|x_{k}-m\right\|
$$

Tomemos $m_{k} \in M$ tal que $1 \leq\left\|x_{k}-m_{k}\right\|<1+2^{-k}$, para todo $k \in \mathbb{N}$. Então, $\left\|x_{k}-m_{k}\right\| \longrightarrow 1$ e, como $\left(x_{k}\right)_{k}$ é limitado, temos que

$$
\left\|m_{k}\right\| \leq\left\|m_{k}-x_{k}\right\|+\left\|x_{k}\right\|<1+2^{-k}+\left\|x_{k}\right\|
$$

Logo, $\left(m_{k}\right)_{k}$ é limitado.

Pelo Corolário 1.1.37, como $X$ é reflexivo, existe $\left(m_{k_{p}}\right)_{p}$ subsequência tal que $m_{k_{p}} \stackrel{w}{\longrightarrow} m$ em $M$. Podemos supor, sem perda de generalidade, que $m_{k} \stackrel{w}{\longrightarrow} m$ em $M$. Consequentemente, $x_{k}-m_{k} \stackrel{w}{\longrightarrow}$ $x-m$ em $M$ e $\left\|x_{k}-m_{k}\right\| \longrightarrow$ 1. Pela Proposição 1.1.34, vem que $\|x-m\| \leq \liminf _{k}\left\|x_{k}-m_{k}\right\|=1$.

Por outro lado,

$$
1=\|\pi(x)\|=\|\pi(x-m)\| \leq\|\pi\|\|x-m\|=\|x-m\|
$$

Assim, $x_{k}-m_{k} \stackrel{w}{\longrightarrow} x-m$ em $X$ e $\left\|x_{k}-m_{k}\right\| \longrightarrow\|x-m\|=1$. Como $X$ tem a KKP, segue que $\left\|x_{k}-m_{k}-(x-m)\right\| \longrightarrow 0$. Dessa forma,

$$
\left\|y_{n_{i_{k}}}-y\right\|=\left\|\pi\left(x_{k}-x\right)\right\|=\| \pi\left(x_{k}-x-\left(m_{k}-m\right)\|\leq\| \pi\|\| x_{k}-m_{k}-(x-m) \| \longrightarrow 0,\right.
$$

contradizendo o fato de que $\left\|y_{n_{i}}-y\right\| \geq \theta$, para todo $i \in \mathbb{N}$.

Logo, $X / M$ tem a KKP. 


\section{Capítulo 3}

\section{Subespaços Fechados do Espaço dos Operadores Compactos com Propriedade Dunford-Pettis Alternativa}

O objetivo deste capítulo é estudar mais exemplos de espaços com a propriedade DP1. Para tal estudaremos subespaços fechados do espaço dos operadores compactos com a propriedade DP1. Na Seção 3.1, trataremos de subespaços fechados do espaço dos operadores compactos em espaços de Banach com a propriedade DP1. Na Seção 3.2, serão vistos os subespaços fechados do espaço dos operadores compactos em espaços de Hilbert. Finalmente, na Seção 3.3, as subálgebras fechadas da álgebra dos operadores compactos em espaços de Banach e de Hilbert.

\subsection{Subespaços Fechados do Espaço dos Operadores Compactos em Espaços de Banach com Propriedade Dunford-Pettis Alterna- tiva}

A proposição seguinte nos dá uma condição necessária para que um subespaço fechado do espaço dos operadores contínuos num espaço de Banach reflexivo tenha a propriedade DP1.

Proposição 3.1.1. Sejam $X, Y$ espaços de Banach reflexivos e $M$ um subespaço fechado de $L(X, Y)$. Se $M$ tem a propriedade $D P 1$, então, para todo $x \in X$ e $g \in Y^{*}$, os operadores avaliação dados por

$$
\begin{aligned}
\delta_{x}: M & \longrightarrow Y & \gamma_{g}: M & \longrightarrow X^{*} \\
S & \longmapsto S(x) & S & \longmapsto S^{*}(g)
\end{aligned}
$$

são operadores DP1.

Demonstração. Como $M$ tem a propriedade DP1, pelo Teorema 2.2 .9 (c), qualquer operador contínuo de $M$ num espaço de Banach reflexivo é um operador DP1.

Assim, como $X^{*}$ e $Y$ são reflexivos, então os operadores $\delta_{x}$ e $\gamma_{g}$ são operadores DP1 para todo $x \in X$ e $g \in Y^{*}$. 
No Teorema 2.2.9, vimos que um espaço de Banach $X$ tem DP1 se, e somente se, para todo espaço de Banach $Y$ e todo operador $T: X \longrightarrow Y$ w-compacto, temos que $T$ é um operador DP1. Veremos que é possível enfraquecer essas hipóteses dependendo do espaço em que estivermos, isto é, será suficiente verificar que os operadores avaliação são operadores DP1. Ademais, veremos que se $X$ é um espaço de Banach com base de Schauder contrátil e $Y$ um espaço de Banach com base de Schauder, então, para cada $M$ subespaço fechado de $K(X, Y)$ que satisfaz à propriedade $\mathcal{P}$ (Definição 3.1.7), é suficiente supor que os operadores avaliação são DP1 para que $M$ possua a propriedade DP1 (Teorema 3.1.14).

O objetivo desta seção é demonstrar esse teorema e os próximos resultados serão necessários para tal. São eles: (i) um resultado equivalente à definição da propriedade DP1 apresentada em 2.2.1; (ii) supondo que os operadores avaliação sejam operadores DP1, apresentamos outros operadores DP1; (iii) a aproximação de operadores compactos por meio de projeções canônicas (operadores de posto finito); (iv) a definição da propriedade $\mathcal{P}$; (v) exemplos de espaços que possuem a propriedade $\mathcal{P}$; e (vi) uma generalização da inequação dada na definição da propriedade $\mathcal{P}$ para somas finitas de operadores.

Começaremos por um resultado equivalente à definição da propriedade DP1, que será utilizado posteriormente.

Proposição 3.1.2. Seja X um espaço de Banach. São equivalentes:

(a) X tem a propriedade DP1;

(b) Para toda sequência $x_{n} \stackrel{w}{\longrightarrow} 0$ em $X, x \in X$, com $\left\|x_{n}+x\right\|=\|x\|=1$, e toda sequência $f_{n} \stackrel{w}{\longrightarrow} 0$ em $X^{*}$, temos que $f_{n}\left(x_{n}\right) \longrightarrow 0$.

Demonstração. (a) $\Rightarrow$ (b) Sejam $x_{n} \stackrel{w}{\longrightarrow} 0$ em $X, x \in X$, com $\left\|x_{n}+x\right\|=\|x\|=1$, e $f_{n} \stackrel{w}{\longrightarrow} 0$ em $X^{*}$.

Temos que $x_{n}+x \stackrel{w}{\longrightarrow} x$ em $X$ e $X$ tem a propriedade DP1, então $f_{n}\left(x_{n}+x\right) \longrightarrow 0$. Como $f_{n} \stackrel{w}{\longrightarrow} 0$, segue que $f_{n}\left(x_{n}\right) \longrightarrow 0$.

(b) $\Rightarrow$ (a) Sejam $x_{n} \stackrel{w}{\longrightarrow} x$ em $X$, com $\left\|x_{n}\right\|=\|x\|=1$, e $f_{n} \stackrel{w}{\longrightarrow} 0$ em $X^{*}$.

Temos que $x_{n}-x \stackrel{w}{\longrightarrow} 0$ e $\left\|\left(x_{n}-x\right)+x\right\|=1$, então, por hipótese, $f_{n}\left(x_{n}-x\right) \longrightarrow 0$. Como $f_{n} \stackrel{w}{\longrightarrow} 0$, segue que $f_{n}\left(x_{n}\right) \longrightarrow 0$.

Supondo que os operadores avaliação são operadores DP1, podemos obter mais operadores com tal propriedade. Isso será visto na próxima proposição. Antes, porém, precisaremos do seguinte lema:

Lema 3.1.3. Sejam $X, Y$ e $Z$ espaços de Banach, $\left(T_{n}\right)_{n} \subset L(X, Y)$ e $T \in L(X, Y)$. Se $T_{n}(x) \longrightarrow$ $T(x)$, para todo $x \in X$, então $T_{n} S \longrightarrow T S$, para todo $S \in K(Z, X)$.

Demonstração. Seja $S \in K(Z, X)$. Para todo $z \in B_{Z}$, temos que $S(z) \in S\left(B_{Z}\right) \subset \overline{S\left(B_{Z}\right)}$ e $\overline{S\left(B_{Z}\right)}$ é compacto, então, pela Proposição 1.1.19,

$$
\sup _{S(z) \in \overline{S\left(B_{Z}\right)}}\left\|T_{n}(S(z))-T(S(z))\right\| \longrightarrow 0
$$


Assim

$$
\left\|T_{n} S-T S\right\|=\sup _{z \in B_{Z}}\left\|\left(T_{n} S-T S\right)(z)\right\| \leq \sup _{S(z) \in \overline{S\left(B_{Z}\right)}}\left\|T_{n}(S(z))-T(S(z))\right\| \longrightarrow 0 .
$$

Logo, $T_{n} S \longrightarrow T S$

Proposição 3.1.4. Sejam $X, Y$ espaços de Banach e $M$ um subespaço fechado de $L(X, Y)$. Se, para todo $x \in X$ e $g \in Y^{*}$, os operadores avaliação dados por

$$
\begin{array}{rlrl}
\delta_{x}: M & \longrightarrow Y & \gamma_{g}: M & \longrightarrow X^{*} \\
S & \longmapsto S(x) & S \longmapsto S^{*}(g)
\end{array}
$$

forem operadores DP1, então, para todo $U \in K(X)$ e $R \in K\left(Y^{*}\right)$, os operadores

$$
\begin{aligned}
& \delta_{U}: M \longrightarrow K(X, Y) \longrightarrow S U \\
& S \longmapsto S
\end{aligned}
$$

$$
\begin{aligned}
\gamma_{R}: M & \longrightarrow K\left(Y^{*}, X^{*}\right) \\
S & \longmapsto S^{*} R
\end{aligned}
$$

são operadores DP1.

Demonstração. Seja $T_{n} \stackrel{w}{\longrightarrow} T$ em $M$, com $\left\|T_{n}\right\|=\|T\|=1$. Como $\delta_{x}$ é um operador DP1, temos que $\left\|\delta_{x}\left(T_{n}\right)-\delta_{x}(T)\right\| \longrightarrow 0$, então $\left\|T_{n}(x)-T(x)\right\| \longrightarrow 0$, para todo $x \in X$. Assim, pelo Lema 3.1.3, para todo $U \in K(X)$, temos que $T_{n} U \longrightarrow T U$. Então, $\left\|\delta_{U}\left(T_{n}\right)-\delta_{U}(T)\right\| \longrightarrow 0$. Logo, $\delta_{U}$ é um operador DP1.

Além disso, como $\gamma_{g}$ é um operador DP1, então $\left\|\gamma_{g}\left(T_{n}\right)-\gamma_{g}(T)\right\| \longrightarrow 0$, isto é, $\left\|T_{n}^{*}(g)-T^{*}(g)\right\| \longrightarrow$ 0, para todo $g \in Y^{*}$. Assim, pelo Lema 3.1.3, para todo $R \in K\left(Y^{*}\right)$, temos que $T_{n}^{*} R \longrightarrow T^{*} R$. Então, $\left\|\gamma_{R}\left(T_{n}\right)-\gamma_{R}(T)\right\| \longrightarrow 0$. Logo, $\gamma_{R}$ é um operador DP1.

Na próxima proposição veremos que podemos aproximar operadores compactos usando projeções em espaços de Banach com base de Schauder. Usaremos esse resultado na demonstração do Teorema 3.1 .14

Proposição 3.1.5. Sejam $X$ um espaço de Banach com base de Schauder contrátil $\left(x_{n}\right)_{n}$ e $Y$ um espaço de Banach com base de Schauder $\left(y_{n}\right)_{n}$. Considere $P_{n}$ e $Q_{n}$ as projeções canônicas sobre as $n$ primeiras coordenadas das bases de $X$ e $Y$ respectivamente. Então, para todo $S \in K(X, Y)$, temos $\left\|S-Q_{n} S P_{n}\right\| \longrightarrow 0$.

Demonstração. Sejam $\varepsilon>0$ e $\alpha$ a constante básica associada à base $\left(x_{n}\right)_{n}$ (Definição 1.2.4).

Como $Q_{n}(y) \longrightarrow y$, para todo $y \in Y$, e $S$ é compacto, segue, pelo Lema 3.1.3, que $Q_{n} S \longrightarrow S$. Logo, existe $n_{1} \in \mathbb{N}$ tal que $\left\|\left(Q_{n}-I\right) S\right\|<\frac{\varepsilon}{2 \alpha}$, para todo $n \geq n_{1}$.

Pela Proposição 1.2.6, como $\left(x_{n}\right)_{n}$ é base contrátil de $X$, então $\left(\psi_{n}\right)_{n}$ é base de Schauder de $X^{*}$. Considerando $P_{n}^{*}: X^{*} \longrightarrow X^{*}$, dado por $P_{n}^{*}(f)=P_{n}^{*}\left(\sum_{i=1}^{\infty} \alpha_{i} \psi_{i}\right)=\sum_{i=1}^{n} \alpha_{i} \psi_{i}$, temos que $P_{n}^{*}(f) \longrightarrow f$, para todo $f \in X^{*}$.

Como $S^{*}$ é compacto (Teorema 1.1.16), segue, pelo Lema 3.1.3, que $P_{n}^{*} S^{*} \longrightarrow S^{*}$. Assim, existe $n_{2} \in \mathbb{N}$ tal que $\left\|\left(P_{n}^{*}-I\right) S^{*}\right\|<\frac{\varepsilon}{2}$, para todo $n \geq n_{2}$. 
Considere $n_{0}=\max \left\{n_{1}, n_{2}\right\}$. Então, para todo $n \geq n_{0}$, temos que

$$
\begin{aligned}
\left\|Q_{n} S P_{n}-S\right\| & =\left\|\left(Q_{n} S P_{n}-S\right)^{*}\right\|=\left\|P_{n}^{*} S^{*} Q_{n}^{*}-S^{*}\right\| \\
& \leq\left\|P_{n}^{*} S^{*} Q_{n}^{*}-P_{n}^{*} S^{*}\right\|+\left\|P_{n}^{*} S^{*}-S^{*}\right\| \\
& \leq\left\|P_{n}^{*}\right\|\left\|S^{*} Q_{n}^{*}-S^{*}\right\|+\left\|\left(P_{n}^{*}-I\right) S^{*}\right\| \\
& =\left\|P_{n}\right\|\left\|\left(Q_{n}-I\right) S\right\|+\left\|\left(P_{n}^{*}-I\right) S^{*}\right\| \\
& <\alpha \frac{\varepsilon}{2 \alpha}+\frac{\varepsilon}{2}=\varepsilon .
\end{aligned}
$$

Logo, $\left\|Q_{n} S P_{n}-S\right\| \longrightarrow 0$.

Proposição 3.1.6. Sejam $X$ um espaço de Banach e $\left(x_{n}\right)_{n}$ uma sequência limitada em $X$. Então, existe $\varphi \in X^{* *}$ um ponto de acumulação fraco-estrela de $\left(x_{n}\right)_{n}$, isto é, $C\left(x_{n}\right) \stackrel{w^{*}}{\longrightarrow} \varphi$, em que $C$ é a aplicação canônica de $X$ em $X^{* *}$.

Demonstração. Como $\left(x_{n}\right)_{n}$ é uma sequência limitada em $X$, podemos supor, sem perda de generalidade, que $\left(x_{n}\right)_{n} \subset B_{X^{* *}}$.

Pelo Teorema de Banach-Alaoglu-Bourbaki (1.1.41), temos que $B_{X^{* *}}$ é $w^{*}$-compacta. Então, segue do Teorema 1.1.29 que toda rede em $B_{X^{* *}}$ tem um ponto de acumulação fraco-estrela. Assim, existe $\varphi \in X^{* *}$ um ponto de acumulação fraco-estrela de $\left(x_{n}\right)_{n}$.

Para verificar que determinados subespaços fechados do espaço dos operadores compactos em espaços de Banach possuem a propriedade DP1, introduziremos a propriedade $\mathcal{P}$. Essa propriedade será definida e estudada nos próximos resultados, como visto em [27].

Definição 3.1.7. Sejam $X$ e $Y$ espaços de Banach com bases de Schauder. Diremos que $M$ um subespaço fechado de $L(X, Y)$ tem a propriedade $\mathcal{P}$ se, para todos $m, n \in \mathbb{N}$ e todos os operadores $T, S \in M$, temos que

$$
\left\|Q_{W} T P_{V}+\left(I_{Y}-Q_{W}\right) S\left(I_{X}-P_{V}\right)\right\| \leq \max \left\{\left\|Q_{W} T P_{V}\right\|,\left\|\left(I_{Y}-Q_{W}\right) S\left(I_{X}-P_{V}\right)\right\|\right\}
$$

sendo $V$ e $W$ subespaços de $X$ e $Y$, respectivamente, gerados pelos primeiros $m$ e $n$ vetores de suas bases, $P_{V}$ e $Q_{W}$ são as projeções canônicas sobre $V$ e $W$ respectivamente, $I_{X}$ e $I_{Y}$ são os operadores identidades em $X$ e $Y$ respectivamente.

As próximas duas proposições nos dão exemplos de espaços de Banach que possuem a propriedade $\mathcal{P}$.

Proposição 3.1.8. Sejam $X$ e $Y$ somas diretas de espaços de Banach de dimensão finita com norma $p$ ( $\ell_{p}$-soma direta) e norma $q$ ( $\ell_{q}$-soma direta) respectivamente, sendo $1<p \leq q<\infty$. Se $M$ é um subespaço fechado de $L(X, Y)$, então $M$ tem a propriedade $\mathcal{P}$.

Demonstração. Sejam $M$ um subespaço fechado de $L(X, Y)$ e $T, S \in M$. Fixemos $m, n \in \mathbb{N}$. Como $X$ e $Y$ têm bases de Schauder, por serem somas diretas de espaços Banach de dimensão finita, consideremos $V, W$ subespaços gerados pelos primeiros $m, n$ vetores das bases de $X, Y$ respectivamente. 
Sejam $P_{V}$ e $Q_{W}$, respectivamente, as projeções de $X$ e $Y$ sobre $V$ e $W, I_{X}$ e $I_{Y}$ são os operadores identidades em $X$ e $Y$ respectivamente.

Considere $Q_{W} T P_{V} \uparrow_{V}: V \longrightarrow W$ e $\left(I_{Y}-Q_{W}\right) S\left(I_{X}-P_{V}\right) \uparrow_{V_{1}}: V_{1} \longrightarrow W_{1}$ operadores contínuos, em que $V_{1}$ e $W_{1}$ são, respectivamente, os subespaços complementares de $V$ e $W$.

Temos que

$$
\begin{gathered}
\left\|Q_{W} T P_{V} \uparrow_{V}\right\|=\sup _{x \in B_{V}}\left\|Q_{W} T P_{V}(x)\right\| \leq \sup _{x \in B_{X}}\left\|Q_{W} T P_{V}(x)\right\|=\left\|Q_{W} T P_{V}\right\| \quad \mathrm{e} \\
\left\|\left(I_{Y}-Q_{W}\right) S\left(I_{X}-P_{V}\right) \uparrow_{V_{1}}\right\|=\sup _{x \in B_{V_{1}}}\left\|\left(I_{Y}-Q_{W}\right) S\left(I_{X}-P_{V}\right)(x)\right\| \\
\leq \sup _{x \in B_{X}}\left\|\left(I_{Y}-Q_{W}\right) S\left(I_{X}-P_{V}\right)(x)\right\|=\left\|\left(I_{Y}-Q_{W}\right) S\left(I_{X}-P_{V}\right)\right\|
\end{gathered}
$$

Segue, do Teorema 1.1.25, que

$\| Q_{W} T P_{V} \uparrow_{V} \oplus\left(I_{Y}-Q_{W}\right) S\left(I_{X}-P_{V}\right)\left\lceil_{V_{1}} \|=\max \left\{\| Q_{W} T P_{V}\left\lceil_{V}\|\|,\left(I_{Y}-Q_{W}\right) S\left(I_{X}-P_{V}\right)\left\lceil_{V_{1}} \|\right\}\right.\right.\right.$

$$
\leq \max \left\{\left\|Q_{W} T P_{V}\right\|,\left\|\left(I_{Y}-Q_{W}\right) S\left(I_{X}-P_{V}\right)\right\|\right\}
$$

Temos que $V \oplus_{p} V_{1}$ e $W \oplus_{q} W_{1}$ são isometricamente isomorfos a $X$ e $Y$ respectivamente, e $Q_{W} T P_{V} \uparrow_{V} \oplus\left(I_{Y}-Q_{W}\right) S\left(I_{X}-P_{V}\right) \uparrow_{V_{1}}$ como operador de $X$ em $Y$ é igual ao operador $Q_{W} T P_{V}+$ $\left(I_{Y}-Q_{W}\right) S\left(I_{X}-P_{V}\right)$. Logo,

$$
\left\|Q_{W} T P_{V}+\left(I_{Y}-Q_{W}\right) S\left(I_{X}-P_{V}\right)\right\| \leq \max \left\{\left\|Q_{W} T P_{V}\right\|,\left\|\left(I_{Y}-Q_{W}\right) S\left(I_{X}-P_{V}\right)\right\|\right\}
$$

Exemplo 3.1.9. Os espaços $L\left(\ell_{p}, \ell_{q}\right)$ e $K\left(\ell_{p}, \ell_{q}\right)$, com $1<p \leq q<\infty$, possuem a propriedade $\mathcal{P}$, uma vez que satisfazem às condições da Proposição 3.1.8.

Proposição 3.1.10. Sejam X um espaço de Banach com base de Schauder e $Y$ uma soma direta de espaços de Banach de dimensão finita com a norma $c_{0}$ (c $c_{0}$-soma direta). Se $M$ é um subespaço fechado de $L(X, Y)$, então $M$ tem a propriedade $\mathcal{P}$.

Demonstração. Sejam $M$ um subespaço fechado de $L(X, Y)$ e $T, S \in M$. Fixemos $m, n \in \mathbb{N}$. Como $Y$ tem base de Schauder, por ser soma direta de espaços Banach de dimensão finita, consideremos $V, W$ subespaços gerados, respectivamente, pelos primeiros $m, n$ vetores das bases de $X, Y$. Sejam $P_{V}$ e $Q_{W}$, respectivamente, projeções de $X$ e $Y$ sobre $V$ e $W, I_{X}$ e $I_{Y}$ são os operadores identidades em $X$ e $Y$ respectivamente.

Consideremos $Q_{W} T P_{V} \uparrow_{V}: V \longrightarrow W$ e $\left(I_{Y}-Q_{W}\right) S\left(I_{X}-P_{V}\right) \uparrow_{V_{1}}: V_{1} \longrightarrow W_{1}$ operadores contínuos, em que $V_{1}$ e $W_{1}$ são os subespaços complementares, respectivamente, de $V$ e $W$.

Seja $x \in B_{X}$. Então, $\left(Q_{W} T P_{V}\right)(x) \in W$ e $\left(I_{Y}-Q_{W}\right) S\left(I_{X}-P_{V}\right)(x) \in W_{1}$. Pela definição da norma $c_{0}$, temos que

$$
\begin{aligned}
\left\|\left(Q_{W} T P_{V}+\left(I_{Y}-Q_{W}\right) S\left(I_{X}-P_{V}\right)\right)(x)\right\| & =\left\|Q_{W} T P_{V}(x)+\left(I_{Y}-Q_{W}\right) S\left(I_{X}-P_{V}\right)(x)\right\| \\
& =\max \left\{\left\|Q_{W} T P_{V}(x)\right\|,\left\|\left(I_{Y}-Q_{W}\right) S\left(I_{X}-P_{V}\right)(x)\right\|\right\} \\
& \leq \max \left\{\left\|Q_{W} T P_{V}\right\|,\left\|\left(I_{Y}-Q_{W}\right) S\left(I_{X}-P_{V}\right)\right\|\right\} .
\end{aligned}
$$


Então,

$$
\begin{aligned}
\left\|Q_{W} T P_{V}+\left(I_{Y}-Q_{W}\right) S\left(I_{X}-P_{V}\right)\right\| & =\sup _{x \in B_{X}}\left\|\left(Q_{W} T P_{V}(x)+\left(I_{Y}-Q_{W}\right) S\left(I_{X}-P_{V}\right)\right)(x)\right\| \\
& \leq \max \left\{\left\|Q_{W} T P_{V}\right\|,\left\|\left(I_{Y}-Q_{W}\right) S\left(I_{X}-P_{V}\right)\right\|\right\} .
\end{aligned}
$$

Exemplo 3.1.11. Os espaços $L\left(X, c_{0}\right)$ e $K\left(X, c_{0}\right)$, em que $X$ é um espaço de Banach com base de Schauder, possuem a propriedade $\mathcal{P}$, como consequência da Proposição 3.1.10.

Dado um subespaço $M$ de $L(X, Y)$ com a propriedade $\mathcal{P}$, em que $X$ e $Y$ têm base de Schauder, podemos obter uma inequação para somas finitas de operadores de $M$ - análoga à inequação dada na definição da propriedade $\mathcal{P}$ (Definição 3.1.7). Tal resultado será estudado nesta proposição:

Proposição 3.1.12. Sejam X, $Y$ espaços de Banach com bases de Schauder e $M$ um subespaço fechado de $L(X, Y)$ com a propriedade $\mathcal{P}$. Sejam $V_{k}$ e $W_{k}$ subespaços de $X$ e $Y$ gerados, respectivamente, pelos primeiros $k$ vetores das bases de $X$ e $Y$. Consideremos $P_{V_{k}}$ e $Q_{W_{k}}$ as projeções canônicas, respectivamente, sobre $V_{k}$ e $W_{k}$, com $P_{V_{0}}=0$ e $Q_{W_{0}}=0$. Então, para toda sequência $\left(T_{k}\right) \subset M$, temos que

$$
\left\|\sum_{k=1}^{n}\left(Q_{W_{k}}-Q_{W_{k-1}}\right) T_{k}\left(P_{V_{k}}-P_{V_{k-1}}\right)\right\| \leq \max _{1 \leq k \leq n}\left\|\left(Q_{W_{k}}-Q_{W_{k-1}}\right) T_{k}\left(P_{V_{k}}-P_{V_{k-1}}\right)\right\| .
$$

Demonstração. Para todo $p \geq 1$ e todo $k \geq p+1$, sendo $p, k \in \mathbb{N}$, temos que

$$
\begin{aligned}
& \left(I_{Y}-Q_{W_{p}}\right)\left(Q_{W_{k}}-Q_{W_{k-1}}\right) T_{k}\left(P_{V_{k}}-P_{V_{k-1}}\right)\left(I_{X}-P_{V_{p}}\right) \\
& =\left(Q_{W_{k}}-Q_{W_{k-1}}-Q_{W_{p}} Q_{W_{k}}+Q_{W_{p}} Q_{W_{k-1}}\right) T_{k}\left(P_{V_{k}}-P_{V_{k-1}}-P_{V_{k}} P_{V_{p}}+P_{V_{k-1}} P_{V_{p}}\right) \\
& =\left(Q_{W_{k}}-Q_{W_{k-1}}\right) T_{k}\left(P_{V_{k}}-P_{V_{k-1}}\right),
\end{aligned}
$$

sendo $I_{X}$ e $I_{Y}$ são os operadores identidades em $X$ e $Y$ respectivamente.

Faremos indução sobre $n$. Para $n=1,2$ é trivial. Para $n=3$, utilizando a definição da propriedade $\mathcal{P}$ e a equação anterior, temos que

$$
\begin{aligned}
\| & \sum_{k=1}^{3}\left(Q_{W_{k}}-Q_{W_{k-1}}\right) T_{k}\left(P_{V_{k}}-P_{V_{k-1}}\right) \| \\
= & \left\|Q_{W_{1}} T_{1} P_{V_{1}}+\left(Q_{W_{2}}-Q_{W_{1}}\right) T_{2}\left(P_{V_{2}}-P_{V_{1}}\right)+\left(Q_{W_{3}}-Q_{W_{2}}\right) T_{3}\left(P_{V_{3}}-P_{V_{2}}\right)\right\| \\
= & \| Q_{W_{1}} T_{1} P_{V_{1}}+\left(I_{Y}-Q_{W_{1}}\right)\left(Q_{W_{2}}-Q_{W_{1}}\right) T_{2}\left(P_{V_{2}}-P_{V_{1}}\right)\left(I_{X}-P_{V_{1}}\right) \\
& +\left(I_{Y}-Q_{W_{1}}\right)\left(Q_{W_{3}}-Q_{W_{2}}\right) T_{3}\left(P_{V_{3}}-P_{V_{2}}\right)\left(I_{X}-P_{V_{1}}\right) \| \\
= & \left\|Q_{W_{1}} T_{1} P_{V_{1}}+\left(I_{Y}-Q_{W_{1}}\right)\left[\left(Q_{W_{2}}-Q_{W_{1}}\right) T_{2}\left(P_{V_{2}}-P_{V_{1}}\right)+\left(Q_{W_{3}}-Q_{W_{2}}\right) T_{3}\left(P_{V_{3}}-P_{V_{2}}\right)\right]\left(I_{X}-P_{V_{1}}\right)\right\| \\
\leq & \max \left\{\left\|Q_{W_{1}} T_{1} P_{V_{1}}\right\|,\left\|\left(I_{Y}-Q_{W_{1}}\right)\left[\left(Q_{W_{2}}-Q_{W_{1}}\right) T_{2}\left(P_{V_{2}}-P_{V_{1}}\right)+\left(Q_{W_{3}}-Q_{W_{2}}\right) T_{3}\left(P_{V_{3}}-P_{V_{2}}\right)\right]\left(I_{X}-P_{V_{1}}\right)\right\|\right\} \\
= & \max \left\{\left\|Q_{W_{1}} T_{1} P_{V_{1}}\right\|,\left\|Q_{W_{2}}\left(I_{Y}-Q_{W_{1}}\right) T_{2}\left(I_{X}-P_{V_{1}}\right) P_{V_{2}}+\left(I_{Y}-Q_{W_{2}}\right) Q_{W_{3}} T_{3} P_{V_{3}}\left(I_{X}-P_{V_{2}}\right)\right\|\right\} \\
\leq & \max \left\{\left\|Q_{W_{1}} T_{1} P_{V_{1}}\right\|, \max \left\{\left\|Q_{W_{2}}\left(I_{Y}-Q_{W_{1}}\right) T_{2}\left(I_{X}-P_{V_{1}}\right) P_{V_{2}}\right\|,\left\|\left(I_{Y}-Q_{W_{2}}\right) Q_{W_{3}} T_{3} P_{V_{3}}\left(I_{X}-P_{V_{2}}\right)\right\|\right\}\right\} \\
= & \max \left\{\left\|Q_{W_{1}} T_{1} P_{V_{1}}\right\|,\left\|Q_{W_{2}}\left(I_{Y}-Q_{W_{1}}\right) T_{2}\left(I_{X}-P_{V_{1}}\right) P_{V_{2}}\right\|,\left\|\left(I_{Y}-Q_{W_{2}}\right) Q_{W_{3}} T_{3} P_{V_{3}}\left(I_{X}-P_{V_{2}}\right)\right\|\right\} \\
= & \max \left\|\left(Q_{W_{k}}-Q_{W_{k-1}}\right) T_{k}\left(P_{V_{k}}-P_{V_{k-1}}\right)\right\| .
\end{aligned}
$$


Supondo que valha para $n$ :

$$
\left\|\sum_{k=1}^{n}\left(Q_{W_{k}}-Q_{W_{k-1}}\right) T_{k}\left(P_{V_{k}}-P_{V_{k-1}}\right)\right\| \leq \max _{1 \leq k \leq n}\left\|\left(Q_{W_{k}}-Q_{W_{k-1}}\right) T_{k}\left(P_{V_{k}}-P_{V_{k-1}}\right)\right\| .
$$

Mostraremos que vale para $n+1$.

$$
\begin{aligned}
& \left\|\sum_{k=1}^{n+1}\left(Q_{W_{k}}-Q_{W_{k-1}}\right) T_{k}\left(P_{V_{k}}-P_{V_{k-1}}\right)\right\| \\
& \leq \max \left\{\left\{\left\|\left(Q_{W_{k}}-Q_{W_{k-1}}\right) T_{k}\left(P_{V_{k}}-P_{V_{k-1}}\right)\right\|: 1 \leq k \leq n-1\right\},\right. \\
& \left.\quad\left\|\left(Q_{W_{n}}-Q_{W_{n-1}}\right) T_{n}\left(P_{V_{n}}-P_{V_{n-1}}\right)+\left(Q_{W_{n+1}}-Q_{W_{n}}\right) T_{n+1}\left(P_{V_{n+1}}-P_{V_{n}}\right)\right\|\right\} \\
& =\max \left\{\left\{\left\|\left(Q_{W_{k}}-Q_{W_{k-1}}\right) T_{k}\left(P_{V_{k}}-P_{V_{k-1}}\right)\right\|: 1 \leq k \leq n-1\right\},\right. \\
& \left.\quad\left\|Q_{W_{n}}\left(I_{Y}-Q_{W_{n-1}}\right) T_{n}\left(I_{X}-P_{V_{n-1}}\right) P_{V_{n}}+\left(I_{Y}-Q_{W_{n}}\right) Q_{W_{n+1}} T_{n+1} P_{V_{n+1}}\left(I_{X}-P_{V_{n}}\right)\right\|\right\} \\
& \leq \max \left\{\left\{\left\|\left(Q_{W_{k}}-Q_{W_{k-1}}\right) T_{k}\left(P_{V_{k}}-P_{V_{k-1}}\right)\right\|: 1 \leq k \leq n-1\right\},\right. \\
& \left.\quad \max \left\{\left\|Q_{W_{n}}\left(I_{Y}-Q_{W_{n-1}}\right) T_{n}\left(I_{X}-P_{V_{n-1}}\right) P_{V_{n}}\right\|,\left\|\left(I_{Y}-Q_{W_{n}}\right) Q_{W_{n+1}} T_{n+1} P_{V_{n+1}}\left(I_{X}-P_{V_{n}}\right)\right\|\right\}\right\} \\
& =\max _{1 \leq k \leq n+1}\left\|\left(Q_{W_{k}}-Q_{W_{k-1}}\right) T_{k}\left(P_{V_{k}}-P_{V_{k-1}}\right)\right\| .
\end{aligned}
$$

Lema 3.1.13. Sejam $X, Y$ espaços de Banach com bases de Schauder e $M$ um subespaço fechado de $L(X, Y)$. Sejam $V_{k}$ e $W_{k}$ subespaços de $X$ e $Y$ gerados respectivamente, pelos primeiros $k$ vetores das bases de $X$ e de $Y$. Consideremos $P_{V_{k}}$ e $Q_{W_{k}}$, respectivamente, as projeções canônicas sobre $V_{k}$ $e W_{k}, I_{X}$ e $I_{Y}$ são os operadores identidades em $X$ e $Y$ respectivamente. Se, para todo $x \in X$ e $g \in Y^{*}$, os operadores avaliação dados por

$$
\begin{aligned}
\delta_{x}: M & \longrightarrow Y & \gamma_{g}: M & \longrightarrow X^{*} \\
S & \longmapsto S(x) & S & \longmapsto S^{*}(g)
\end{aligned}
$$

são operadores DP1, então, para toda sequência $T_{n} \stackrel{w}{\longrightarrow} T$ em $M$, com $\left\|T_{n}\right\|=\|T\|=1$, temos que $\left\|T_{n}-\left(I_{Y}-Q_{W_{k}}\right) T_{n}\left(I_{X}-P_{V_{k}}\right)\right\| \longrightarrow 0$.

Demonstração. Sejam $\left(x_{n}\right)_{n}$ e $\left(y_{n}\right)_{n}$ bases de Schauder de $X$ e $Y$ respectivamente, e $\alpha$ a constante básica associada à base de $X$. Seja $T_{n} \stackrel{w}{\longrightarrow} T$ em $M$, com $\left\|T_{n}\right\|=\|T\|=1$.

Fixemos $k \in \mathbb{N}$. Como $P_{V_{k}}$ e $Q_{W_{k}}$ são operadores de posto finito, então são compactos e $Q_{W_{k}}^{*}$ também é compacto (Teorema 1.1.16). Segue, da Proposição 3.1.4, que os operadores

$$
\begin{array}{rlrl}
\delta_{P_{V_{k}}}: M & \longrightarrow K(X, Y) & \gamma_{Q_{W_{k}}^{*}}: M & \longrightarrow K\left(Y^{*}, X^{*}\right) \\
S & \longmapsto S P_{V_{k}} & S & \longmapsto S^{*} Q_{W_{k}}^{*}
\end{array}
$$

são operadores DP1. Logo,

$$
\begin{aligned}
\delta_{P_{V_{k}}}\left(T_{n}\right) \longrightarrow 0 & \Longrightarrow T_{n} P_{V_{k}} \longrightarrow 0 \Longrightarrow\left\|T_{n} P_{V_{k}}\right\| \longrightarrow 0 \mathrm{e} \\
\gamma_{Q_{W_{k}}^{*}}\left(T_{n}\right) \longrightarrow 0 & \Longrightarrow T_{n}^{*} Q_{W_{k}}^{*} \longrightarrow 0 \Longrightarrow\left\|T_{n}^{*} Q_{W_{k}}\right\|=\left\|Q_{W_{k}} T_{n}\right\| \longrightarrow 0
\end{aligned}
$$


Como $\alpha$ é a constante básica associada a $\left(x_{n}\right)_{n}$, temos que

$$
\left\|I_{X}-P_{V_{k}}\right\| \leq\left\|I_{X}\right\|+\left\|P_{V_{k}}\right\| \leq 1+\alpha .
$$

Logo,

$$
\begin{aligned}
\left\|T_{n}-\left(I_{Y}-Q_{W_{k}}\right) T_{n}\left(I_{X}-P_{V_{k}}\right)\right\| & =\left\|T_{n}-\left(T_{n}-Q_{W_{k}} T_{n}\right)\left(I_{X}-P_{V_{k}}\right)\right\| \\
& =\left\|T_{n}-T_{n}+T_{n} P_{V_{k}}+Q_{W_{k}} T_{n}\left(I_{X}-P_{V_{k}}\right)\right\| \\
& \leq\left\|T_{n} P_{V_{k}}\right\|+\left\|Q_{W_{k}} T_{n}\right\|\left\|I_{X}-P_{V_{k}}\right\| \\
& \leq\left\|T_{n} P_{V_{k}}\right\|+\left\|Q_{W_{k}} T_{n}\right\|(1+\alpha) \longrightarrow 0 .
\end{aligned}
$$

O teorema a seguir nos dá condições suficientes para que um subespaço fechado do espaço dos operadores compactos num espaço de Banach possua a propriedade DP1. A demonstração utilizará os resultados estudados ao longo desta seção.

Teorema 3.1.14. Sejam $X$ um espaço de Banach com base de Schauder contrátil, $Y$ um espaço de Banach com base de Schauder e $M$ subespaço fechado de $K(X, Y)$ satisfazendo à propriedade $\mathcal{P}$. Se, para todos $x \in X$ e $g \in Y^{*}$, os operadores avaliação

$$
\begin{aligned}
& \delta_{x}: M \longrightarrow Y \\
& \gamma_{g}: M \longrightarrow X^{*} \\
& S \mapsto S(x) \\
& S \mapsto S^{*}(g)
\end{aligned}
$$

são operadores DP1, então $M$ tem a propriedade DP1.

Demonstração. Sejam $\left(x_{n}\right)_{n}$ e $\left(y_{n}\right)_{n}$ bases de Schauder, respectivamente, de $X$ e $Y$, e $\alpha$ e $\beta$ as constantes básicas associadas, respectivamente, às bases de $X$ e $Y$. Considerem $I_{X}$ e $I_{Y}$ os operadores identidades em $X$ e $Y$ respectivamente.

Suponhamos que $M$ não tem a propriedade DP1. Então existem $T_{n} \stackrel{w}{\longrightarrow} 0$ em $M, T \in M$, com $\left\|T_{n}+T\right\|=\|T\|=1$, e $f_{n} \stackrel{w}{\longrightarrow} 0$ em $M^{*}$ tal que $f_{n}\left(T_{n}\right) \nrightarrow 0$, isto é, existe $r>0$ tal que, para todo $n \in \mathbb{N}$,

$$
\left|f_{n}\left(T_{n}\right)\right| \geq r
$$

Construíremos uma subsequência de $\left(f_{n}\right)_{n}$ que não converge fracamente a zero. Isso nos levará a uma contradição, pois $\left(f_{n}\right)_{n}$ converge fracamente a zero.

Consideremos $f_{\sigma(1)}=f_{1}, T_{\sigma(1)}=T_{1}$. Como $T_{\sigma(1)} \in K(X, Y)$, pela Proposição 3.1.5, existe $p_{1} \in \mathbb{N}$ tal que

$$
\left\|T_{\sigma(1)}-Q_{W_{1}} T_{\sigma(1)} P_{V_{1}}\right\|<\frac{1}{2}
$$

em que $V_{1}=\left[x_{1}, \ldots, x_{p_{1}}\right], W_{1}=\left[y_{1}, \ldots, y_{p_{1}}\right], P_{V_{1}}$ e $Q_{W_{1}}$ são, respectivamente, as projeções de $X, Y$ sobre $V_{1}, W_{1}$. 
Segue, pelo Lema 3.1.13, que, para um $n$ suficientemente grande,

$$
\left\|T_{n}-\left(I_{Y}-Q_{W_{1}}\right) T_{n}\left(I_{X}-P_{V_{1}}\right)\right\|<\frac{1}{2^{4}} .
$$

Como $T_{n} \stackrel{w}{\longrightarrow} 0$ e $f_{n} \stackrel{w}{\longrightarrow} 0$, temos que

$$
f_{\sigma(1)}\left(T_{n}\right) \longrightarrow 0 \Longrightarrow\left|f_{\sigma(1)}\left(T_{n}\right)\right|<\frac{r}{2^{3}} \quad \text { e } \quad f_{n}\left(T_{\sigma(1)}\right) \longrightarrow 0 \Longrightarrow\left|f_{n}\left(T_{\sigma(1)}\right)\right|<\frac{r}{2^{3}} .
$$

Escolhemos $\sigma(2)>\sigma(1)$ satisfazendo a essas condições, ou seja,

$$
\begin{array}{r}
\| T_{\sigma(2)}-\left(I_{Y}-Q_{W_{1}}\right) T_{\sigma(2)}\left(I_{X}-P_{V_{1}}\right)||<\frac{1}{2^{4}}, \\
\left|f_{\sigma(1)}\left(T_{\sigma(2)}\right)\right|<\frac{r}{2^{3}}, \\
\left|f_{\sigma(2)}\left(T_{\sigma(1)}\right)\right|<\frac{r}{2^{3}} \quad \mathrm{e} \\
\left|f_{\sigma(2)}\left(T_{\sigma(2)}\right)\right| \geq r .
\end{array}
$$

Como $T_{\sigma(2)}$ é compacto, então $\left(I-Q_{W_{1}}\right) T_{\sigma(2)}\left(I-P_{V_{1}}\right)$ é um operador compacto. Pela Proposição 3.1.5, existe $p_{2}>p_{1}$ tal que

$$
\left\|\left(I_{Y}-Q_{W_{1}}\right) T_{\sigma(2)}\left(I_{X}-P_{V_{1}}\right)-Q_{W_{2}}\left(I_{Y}-Q_{W_{1}}\right) T_{\sigma(2)}\left(I_{X}-P_{V_{1}}\right) P_{V_{2}}\right\|<\frac{1}{2^{4}},
$$

em que $V_{2}=\left[x_{1}, \ldots, x_{p_{2}}\right], W_{2}=\left[y_{1}, \ldots, y_{p_{2}}\right], P_{V_{2}}$ e $Q_{W_{2}}$ são, respectivamente, as projeções de $X$ e $Y$ sobre $V_{2}$ e $W_{2}$.

Observemos que $p_{2}>p_{1}$, então $Q_{W_{2}} Q_{W_{1}}=Q_{W_{1}} Q_{W_{2}}=Q_{W_{1}}$ e $P_{V_{2}} P_{V_{1}}=P_{V_{1}} P_{V_{2}}=P_{V_{1}}$, donde $Q_{W_{2}}\left(I_{Y}-Q_{W_{1}}\right) T_{\sigma(2)}\left(I_{X}-P_{V_{1}}\right) P_{V_{2}}=\left(Q_{W_{2}}-Q_{W_{2}} Q_{W_{1}}\right) T_{\sigma(2)}\left(P_{V_{2}}-P_{V_{1}} P_{V_{2}}\right)=\left(Q_{W_{2}}-\right.$ $\left.Q_{W_{1}}\right) T_{\sigma(2)}\left(P_{V_{2}}-P_{V_{1}}\right)$. Assim:

$$
\begin{aligned}
& \left\|T_{\sigma(2)}-\left(Q_{W_{2}}-Q_{W_{1}}\right) T_{\sigma(2)}\left(P_{V_{2}}-P_{V_{1}}\right)\right\| \\
= & \left\|T_{\sigma(2)}-\left(I_{Y}-Q_{W_{1}}\right) T_{\sigma(2)}\left(I_{X}-P_{V_{1}}\right)+\left(I_{Y}-Q_{W_{1}}\right) T_{\sigma(2)}\left(I_{X}-P_{V_{1}}\right)-\left(Q_{W_{2}}-Q_{W_{1}}\right) T_{\sigma(2)}\left(P_{V_{2}}-P_{V_{1}}\right)\right\| \\
\leq & \left\|T_{\sigma(2)}-\left(I_{Y}-Q_{W_{1}}\right) T_{\sigma(2)}\left(I_{X}-P_{V_{1}}\right)\right\|+\left\|\left(I_{Y}-Q_{W_{1}}\right) T_{\sigma(2)}\left(I_{X}-P_{V_{1}}\right)-\left(Q_{W_{2}}-Q_{W_{1}}\right) T_{\sigma(2)}\left(P_{V_{2}}-P_{V_{1}}\right)\right\| \\
< & \frac{1}{2^{4}}+\frac{1}{2^{4}}=\frac{1}{2^{3}} .
\end{aligned}
$$

Suponhamos que escolhemos $p_{1}<p_{2}<\cdots<p_{k} \in \mathbb{N}$ e os $k$ primeiros índices da subsequência, denotados por $\sigma(1)<\sigma(2)<\cdots<\sigma(k) \in \mathbb{N}$, tais que

$$
\begin{aligned}
& \left\|T_{\sigma(i)}-\left(Q_{W_{i}}-Q_{W_{i-1}}\right) T_{\sigma(i)}\left(P_{V_{i}}-P_{V_{i-1}}\right)\right\|<\frac{1}{2^{i+1}}, \\
& \left|f_{\sigma(i)}\left(T_{\sigma(i)}\right)\right| \geq r, \quad 1 \leq i \leq k, \\
& \left|f_{\sigma(i)}\left(T_{\sigma(k)}\right)\right|<\frac{r}{2^{k+1}} \quad \mathrm{e} \\
& \left|f_{\sigma(k)}\left(T_{\sigma(i)}\right)\right|<\frac{r}{2^{k+1}}, \quad i<n,
\end{aligned}
$$

sendo $V_{i}=\left[x_{1}, \cdots, x_{p_{i}}\right]$ e $W_{i}=\left[y_{1}, \cdots, y_{p_{i}}\right]$. 
Pelo Lema 3.1.13, para um $p$ suficientemente grande, temos que

$$
\left\|T_{p}-\left(I_{Y}-Q_{W_{k}}\right) T_{p}\left(I_{X}-P_{V_{k}}\right)\right\|<\frac{1}{2^{(k+1)+2}} .
$$

Como $T_{p} \stackrel{w}{\longrightarrow} 0$ e $f_{p} \stackrel{w}{\longrightarrow} 0$, para $i \leq p$, temos que

$$
f_{\sigma_{i}}\left(T_{p}\right) \longrightarrow 0 \Longrightarrow\left|f_{\sigma_{i}}\left(T_{p}\right)\right|<\frac{r}{2^{k+2}} \quad \text { e } \quad f_{p}\left(T_{\sigma_{i}}\right) \longrightarrow 0 \Longrightarrow\left|f_{p}\left(T_{\sigma_{i}}\right)\right|<\frac{r}{2^{k+2}}
$$

Escolhemos $\sigma(k+1)>\sigma(k)$ satisfazendo às condições anteriores, ou seja,

$$
\begin{aligned}
\left\|T_{\sigma(k+1)}-\left(I_{Y}-Q_{W_{k}}\right) T_{\sigma(k+1)}\left(I_{X}-P_{V_{k}}\right)\right\| & <\frac{1}{2^{(k+1)+2}}, \\
\left|f_{\sigma_{1}}\left(T_{\sigma(k+1)}\right)\right| & <\frac{r}{2^{k+2}}, \\
\left|f_{\sigma(i)}\left(T_{\sigma_{i}}\right)\right| & <\frac{r}{2^{k+2}} \quad \text { e } \\
\left|f_{\sigma(k+1)}\left(T_{\sigma(2)}\right)\right| & \geq r,
\end{aligned} \quad i \leq k .
$$

Pela Proposição 3.1.5, podemos aproximar o operador compacto $\left(I_{Y}-Q_{W_{k}}\right) T_{\sigma(k+1)}\left(I_{X}-P_{V_{k}}\right)$, isto é, existe $p_{k+1}>p_{k}$ tal que

$$
\left\|\left(I_{Y}-Q_{W_{k}}\right) T_{\sigma(k+1)}\left(I_{X}-P_{V_{k}}\right)-Q_{W_{k+1}}\left(I_{Y}-Q_{W_{k}}\right) T_{\sigma(k+1)}\left(I_{X}-P_{V_{k}}\right) P_{V_{k+1}}\right\|<\frac{1}{2^{(k+1)+2}},
$$

em que $V_{k+1}=\left[x_{1}, \ldots, x_{p_{k+1}}\right], W_{k+1}=\left[y_{1}, \ldots, y_{p_{k+1}}\right]$.

Observemos novamente que $Q_{W_{k+1}} Q_{W_{k}}=Q_{W_{k}} Q_{W_{k+1}}=Q_{W_{k}}$ e $P_{V_{k+1}} P_{V_{k}}=P_{V_{k}} P_{V_{k+1}}=P_{V_{k}}$. Assim:

$$
\begin{aligned}
& \left\|T_{\sigma(k+1)}-\left(Q_{W_{k+1}}-Q_{W_{k}}\right) T_{\sigma(k+1)}\left(P_{V_{k+1}}-P_{V_{k}}\right)\right\| \\
\leq & \left\|T_{\sigma(k+1)}-\left(I_{Y}-Q_{W_{k}}\right) T_{\sigma(k+1)}\left(I_{X}-P_{V_{k}}\right)\right\| \\
& +\left\|\left(I_{Y}-Q_{W_{k}}\right) T_{\sigma(k+1)}\left(I_{X}-P_{V_{k}}\right)-\left(Q_{W_{k+1}}-Q_{W_{k}}\right) T_{\sigma(k+1)}\left(P_{V_{k+1}}-P_{V_{k}}\right)\right\| \\
< & \frac{1}{2^{k+3}}+\frac{1}{2^{k+3}}=\frac{1}{2^{k+2}} .
\end{aligned}
$$

Além disso,

$$
\begin{array}{rlr}
\left|f_{\sigma(k+1)}\left(T_{\sigma(k+1)}\right)\right| & \geq r \\
\left|f_{\sigma(k+1)}\left(T_{\sigma(i)}\right)\right| & <\frac{r}{2^{k+1}} \quad \mathrm{e} & \\
\left|f_{\sigma(i)}\left(T_{\sigma(k+1)}\right)\right| & <\frac{r}{2^{k+1}}, & i<k+1 .
\end{array}
$$

Dessa forma, construímos $\left(f_{\sigma(n)}\right)_{n}$ uma subsequência de $\left(f_{n}\right)_{n}$. Mostraremos que $f_{\sigma(n)} \stackrel{w}{\rightarrow} 0$. Primeiramente, vejamos que $S_{n}=\sum_{k=1}^{n} T_{\sigma(k)}$ é limitado. Lembremos que $\alpha$ e $\beta$ são as constantes 
associadas, respectivamente, às bases de $X$ e $Y$. Consideremos $P_{V_{0}}=0$ e $Q_{W_{0}}=0$. Pela Proposição 3.1.12, segue que

$$
\begin{aligned}
& \left\|\sum_{k=1}^{n}\left(Q_{W_{k}}-Q_{W_{k-1}}\right) T_{\sigma(k)}\left(P_{V_{k}}-P_{V_{k-1}}\right)\right\| \leq \max _{1 \leq k \leq n}\left\|\left(Q_{W_{k}}-Q_{W_{k-1}}\right) T_{\sigma(k)}\left(P_{V_{k}}-P_{V_{k-1}}\right)\right\| \\
& \leq\left(\max _{1 \leq k \leq n}\left\|Q_{W_{k}}\right\|+\max _{1 \leq k \leq n}\left\|Q_{W_{k-1}}\right\|\right) \max _{1 \leq k \leq n}\left\|T_{\sigma(k)}\right\|\left(\max _{1 \leq k \leq n}\left\|P_{V_{k}}\right\|+\max _{1 \leq k \leq n}\left\|P_{V_{k-1}}\right\|\right) \\
& \leq 4 \alpha \beta \max _{1 \leq k \leq n}\left\|T_{\sigma(k)}\right\| .
\end{aligned}
$$

Como $T_{\sigma(k)} \stackrel{w}{\longrightarrow} 0$ em $M$, então $\left(\left\|T_{\sigma(k)}\right\|\right)_{n}$ é limitada, isto é, existe $D>0$ tal que $\left\|T_{\sigma(k)}\right\| \leq D$, para todo $n \in \mathbb{N}$.

Temos ainda que

$$
\begin{aligned}
\left\|\sum_{k=1}^{n} T_{\sigma(k)}\right\| & \leq\left\|\sum_{k=1}^{n} T_{\sigma(k)}-\sum_{k=1}^{n}\left(Q_{W_{k}}-Q_{W_{k-1}}\right) T_{\sigma(k)}\left(P_{V_{k}}-P_{V_{k-1}}\right)\right\| \\
& +\left\|\sum_{k=1}^{n}\left(Q_{W_{k}}-Q_{W_{k-1}}\right) T_{\sigma(k)}\left(P_{V_{k}}-P_{V_{k-1}}\right)\right\| \\
& \leq \sum_{k=1}^{n}\left\|T_{\sigma(k)}-\left(Q_{W_{k}}-Q_{W_{k-1}}\right) T_{\sigma(k)}\left(P_{V_{k}}-P_{V_{k-1}}\right)\right\| \\
& +\left\|\sum_{k=1}^{n}\left(Q_{W_{k}}-Q_{W_{k-1}}\right) T_{\sigma(k)}\left(P_{V_{k}}-P_{V_{k-1}}\right)\right\| \\
& \leq \sum_{k=1}^{n} \frac{1}{2(k+1)}+4 \alpha \beta D .
\end{aligned}
$$

Assim, $\left(S_{n}\right)_{n}$ é uma sequência limitada em $M$. Pela Proposição 3.1.6, existe $\varphi \in M^{* *}$ um ponto de acumulação fraco-estrela de $\left(S_{m}\right)$, isto é, $C\left(S_{m}\right) \stackrel{w^{*}}{\longrightarrow} \varphi$ em $M^{* *}$. Então

$$
\varphi_{m}=C\left(S_{m}\right) \stackrel{w^{*}}{\longrightarrow} \varphi \mathrm{em} M^{* *} \Longleftrightarrow C\left(S_{m}\right)(f) \longrightarrow \varphi(f), \quad \forall f \in M^{*} .
$$

Fixemos $n \in \mathbb{N}$,

$$
C\left(S_{m}\right)\left(f_{\sigma(n)}\right) \longrightarrow \varphi\left(f_{\sigma(n)}\right) \Rightarrow f_{\sigma(n)}\left(S_{m}\right) \longrightarrow \varphi\left(f_{\sigma(n)}\right)
$$


Para $p>n$ e usando (3.1) e (3.2), temos que

$$
\begin{aligned}
\left|f_{\sigma(n)}\left(\sum_{k=1}^{p} T_{\sigma(k)}\right)\right| & =\left|\sum_{k=1}^{p} f_{\sigma(n)}\left(T_{\sigma(k)}\right)\right|=\left|f_{\sigma(n)}\left(T_{\sigma(n)}\right)+\sum_{\substack{k=1 \\
k \neq n}}^{p} f_{\sigma(n)}\left(T_{\sigma(k)}\right)\right| \\
& \geq\left|f_{\sigma(n)}\left(T_{\sigma(n)}\right)\right|-\left|\sum_{\substack{k=1 \\
k \neq n}}^{p} f_{\sigma(n)}\left(T_{\sigma(k)}\right)\right| \geq\left|f_{\sigma(n)}\left(T_{\sigma(n)}\right)\right|-\sum_{\substack{k=1 \\
k \neq n}}^{p}\left|f_{\sigma(n)}\left(T_{\sigma(k)}\right)\right| \\
& \geq r-\sum_{k=1}^{n-1}\left|f_{\sigma(n)}\left(T_{\sigma(k)}\right)\right|-\sum_{k=n+1}^{p}\left|f_{\sigma(n)}\left(T_{\sigma(k)}\right)\right| \geq r-\sum_{k=1}^{n-1} \frac{r}{2^{n+1}}-\sum_{k=n+1}^{p} \frac{r}{2^{k+1}} \\
& =r\left(1-\left(\sum_{k=1}^{n-1} \frac{1}{2^{n+1}}-\sum_{k=n+1}^{p} \frac{1}{2^{k+1}}\right)\right) \geq r\left(1-\sum_{k=1}^{p} \frac{1}{2^{k+1}}\right) \\
& \geq r\left(1-\sum_{k=1}^{\infty} \frac{1}{2^{k+1}}\right)=r\left(1-\frac{1}{2}\right)=\frac{r}{2} .
\end{aligned}
$$

Assim,

$$
\left|f_{\sigma(n)}\left(S_{m_{k}}\right)-\varphi\left(f_{\sigma(n)}\right)\right| \geq\left|f_{\sigma(n)}\left(S_{m_{k}}\right)\right|-\left|\varphi\left(f_{\sigma(n)}\right)\right| \geq \frac{r}{2}-\left|\varphi\left(f_{\sigma(n)}\right)\right| .
$$

Então, para todo $n \in \mathbb{N}$, temos que

$$
\frac{r}{2}-\left|\varphi\left(f_{\sigma(n)}\right)\right| \leq\left|f_{\sigma(n)}\left(S_{m_{k}}\right)-\varphi\left(f_{\sigma(n)}\right)\right| \longrightarrow 0 \Longrightarrow\left|\varphi\left(f_{\sigma(n)}\right)\right| \geq \frac{r}{2}, \quad \forall n \in \mathbb{N} .
$$

Logo, $\varphi\left(f_{\sigma(n)}\right) \nrightarrow 0$ e, portanto, $f_{\sigma(n)} \stackrel{w}{\leftrightarrow} 0$.

Como $\left(f_{n}\right)_{n}$ converge fracamente a zero chegamos a uma contradição. Logo, $M$ tem a propriedade DP1.

Como consequência da Proposição 3.1.1 e do Teorema 3.1.14, obtemos o principal resultado desta seção: a caracterização dos subespaços fechados do espaço dos operadores compactos em espaços de Banach.

Corolário 3.1.15. Sejam X, Y espaços de Banach reflexivos com bases de Schauder e $M$ subespaço fechado de $K(X, Y)$ satisfazendo à propriedade $\mathcal{P}$. Então, $M$ tem a propriedade DP1 se, e somente se, para todo $x \in X$ e $g \in Y^{*}$, os operadores avaliação

$$
\begin{array}{rlrl}
\delta_{x}: M & \longrightarrow Y & \gamma_{g}: M & \longrightarrow X^{*} \\
S & \mapsto S(x) & S \mapsto S^{*}(g)
\end{array}
$$

são operadores DP1.

Demonstração. $\Rightarrow$ Segue da Proposição 3.1.1.

$\Leftarrow$ Como $X$ é reflexivo, pelo Teorema 1.2.8, a sua base de Schauder é contrátil. Segue do Teorema 3.1.14 que $M$ tem a propriedade DP1. 


\subsection{Subespaços Fechados do Espaço dos Operadores Compactos em Espaços de Hilbert com Propriedade Dunford-Pettis Alterna- tiva}

Nosso objetivo nesta seção será estudar um resultado análogo ao Corolário 3.1.15 em espaços de Hilbert. Em outras palavras, a demonstração do principal resultado desta seção utilizará a mesma construção da demonstração do Teorema 3.1.14. Para tal estudaremos resultados em espaços de Hilbert análogos aos resultados estudados em espaços de Banach.

Apesar da semelhança entre as demonstrações, uma diferença a ser notada está nos operadores avaliação: o operador transposto substituirá o operador adjunto. Assim, o operador avaliação $\gamma$ terá contradomínio no próprio espaço de Hilbert, e não no seu dual, como ocorre nos espaços de Banach. É válido lembrar que todo espaço de Hilbert é isometricamente isomorfo ao seu dual e que, como mencionado na Seção 1.3, os operadores transposto e adjunto estão relacionados em espaços de Hilbert. Veremos também que todo espaço de Hilbert satisfaz a uma equação análoga à equação da definição da propriedade $\mathcal{P}$ para espaços de Banach.

Sabemos que todo espaço de Hilbert possui um sistema ortonormal completo não necessariamente enumerável. Quando o espaço de Hilbert é separável, esse sistema ortonormal completo é enumerável e tem papel semelhante à base de Schauder em espaços de Banach. Apesar disso, apresentaremos um resultado para espaços de Hilbert análogo ao Corolário 3.1.15, sem a necessidade de que o espaço de Hilbert seja separável.

A proposição a seguir nos dá uma condição necessária para que um subespaço fechado do espaço dos operadores contínuos num espaço de Hilbert tenha a propriedade DP1. (Confronte com a Proposição 3.1.1)

Proposição 3.2.1. Sejam $H$ um espaço de Hilbert e $M$ um subespaço fechado de $L(H)$. Se $M$ tem a propriedade DP1, então, para todo $h \in H$, os operadores avaliação

$$
\begin{array}{rr}
\delta_{h}: M \longrightarrow H & \gamma_{h}: M \longrightarrow H \\
S \mapsto S(h) & S \mapsto S^{t}(h)
\end{array}
$$

são operadores DP1.

Demonstração. Como $M$ tem a propriedade DP1, pelo Teorema 2.2 .9 (c), qualquer operador contínuo de $M$ em um espaço de Banach reflexivo é um operador DP1.

Assim, como $H$ é reflexivo, então os operadores $\delta_{h}$ e $\gamma_{h}$ são operadores DP1, para todo $h \in H$.

Na próxima proposição, veremos que, ao supor que os operadores avaliação são operadores DP1, obtemos mais operadores com tal propriedade.

Proposição 3.2.2. Sejam $H$ um espaço de Hilbert e $M$ um subespaço fechado de $L(H)$. Se, para 
todo $h \in H$, os operadores avaliação

$$
\begin{array}{r}
\delta_{h}: M \longrightarrow H \\
S \mapsto S(h)
\end{array}
$$

$$
\begin{aligned}
\gamma_{h}: M & \longrightarrow H \\
S & \mapsto S^{t}(h)
\end{aligned}
$$

forem operadores DP1, então, para todo $U \in K(H)$, os operadores

$$
\begin{aligned}
\delta_{U}: M & \longrightarrow K(H) \\
S & \longmapsto S U
\end{aligned}
$$

$$
\begin{aligned}
\gamma_{U^{t}}: M & \longrightarrow K(H) \\
S & \longmapsto S^{t} U^{t}
\end{aligned}
$$

são operadores DP1.

Demonstração. Seja $T_{n} \stackrel{w}{\longrightarrow} T$ em $M$, com $\left\|T_{n}\right\|=\|T\|=1$. Como $\delta_{h}$ é operador DP1, temos que $\left\|\delta_{h}\left(T_{n}\right)-\delta_{h}(T)\right\| \longrightarrow 0$, então $\left\|T_{n}(h)-T(h)\right\| \longrightarrow 0$, para todo $h \in H$. Assim, pelo Lema 3.1.3, para todo $U \in K(H)$, temos que $T_{n} U \longrightarrow T U$. Então, $\left\|\delta_{U}\left(T_{n}\right)-\delta_{U}(T)\right\| \longrightarrow 0$. Logo, $\delta_{U}$ é um operador DP1.

Além disso, como $\gamma_{h}$ é um operador DP1, então $\left\|\gamma_{h}\left(T_{n}\right)-\gamma_{h}(T)\right\| \longrightarrow 0$, isto é, $\left\|T_{n}^{t}(h)-T^{t}(h)\right\| \longrightarrow$ 0 , para todo $h \in H$. Assim, pelo Lema 3.1.3, para todo $U \in K(H)$, temos que $T_{n}^{t} U \longrightarrow T^{t} U$. Então, $\left\|\gamma_{U^{t}}\left(T_{n}\right)-\gamma_{U^{t}}(T)\right\| \longrightarrow 0$. Logo, $\gamma_{U^{t}}$ é um operador DP1.

Na Proposição 3.1.5, vimos que podemos aproximar operadores compactos em espaços de Banach por meio de projeções. Um resultado análogo para operadores compactos em espaços de Hilbert será estudado a seguir.

Proposição 3.2.3. Se $H$ é um espaço de Hilbert e $S \in K(H)$, então, para todo $\varepsilon>0$, existe um subespaço de dimensão finita $V \subset H$ tal que $\left\|S-P_{V} S P_{V}\right\|<\varepsilon$, sendo $P_{V}$ a projeção ortogonal sobre $V$.

Demonstração. Sejam $H$ um espaço de Hilbert, $S \in K(H)$ e $\varepsilon>0$. Como $S$ é compacto, segue que $S\left(B_{H}\right)$ é relativamente compacto. Então, existem $\left\{x_{i}: 1 \leq i \leq n\right\}$ tais que $S\left(B_{H}\right) \subset \bigcup_{i=1}^{n} B_{\frac{\varepsilon}{4}}\left(x_{i}\right)$.

Para cada $h \in B_{H}$, temos que $S(h) \in \bigcup_{i=1}^{n} B_{\frac{\varepsilon}{4}}\left(x_{i}\right)$. Então, existe $j \in\{1, \ldots, n\}$ tal que $S(h) \in$ $B_{\frac{\varepsilon}{4}}\left(x_{j}\right)$, isto é, $\left\|S(h)-x_{j}\right\|<\frac{\varepsilon}{4}$.

Analogamente, como $S^{t} \in K(H)$, segue que $S^{t}\left(B_{H}\right)$ é relativamente compacto. Então, existem $\left\{y_{i}: 1 \leq i \leq m\right\}$ tais que $S^{t}\left(B_{H}\right) \subset \bigcup_{i=1}^{m} B_{\frac{\varepsilon}{4}}\left(y_{i}\right)$.

Para cada $h \in B_{H}$, temos que $S(h) \in \bigcup_{i=1}^{m} B_{\frac{\varepsilon}{4}}\left(y_{i}\right)$. Então, existe $k \in\{1, \ldots, m\}$ tal que $S(h) \in B_{\frac{\varepsilon}{4}}\left(y_{k}\right)$, isto é, $\left\|S(h)-y_{k}\right\|<\frac{\varepsilon}{4}$.

Consideremos $V=\left[x_{1}, \cdots, x_{n}, y_{1}, \cdots, y_{m}\right]$ e $P_{V}: H \longrightarrow V$ a projeção ortogonal de $H$ sobre $V$. Pelo Teorema 1.3.1, sabemos que $\left\|P_{V}\right\|=1$. Então, para todo $h \in B_{H}$, temos que

$$
\left\|P_{V} S(h)-x_{i}\right\|=\left\|P_{V} S(h)-P_{V}\left(x_{i}\right)\right\| \leq\left\|P_{V}\right\|\left\|S(h)-x_{i}\right\|=\left\|S(h)-x_{i}\right\|, \quad \forall 1 \leq i \leq n .
$$




$$
\left\|S(h)-P_{V} S(h)\right\| \leq\left\|S(h)-x_{j}\right\|+\left\|x_{j}-P_{V} S(h)\right\| \leq 2\left\|S(h)-x_{j}\right\|<\frac{\varepsilon}{2} .
$$

Analogamente,

$$
\begin{gathered}
\left\|P_{V} S^{t}(h)-y_{i}\right\|=\left\|P_{V} S^{t}(h)-P_{V}\left(y_{i}\right)\right\| \leq\left\|P_{V}\right\|\left\|S^{t}(h)-y_{i}\right\|=\left\|S^{t}(h)-y_{i}\right\|, \quad \forall 1 \leq i \leq m . \\
\left\|S^{t}(h)-P_{V} S^{t}(h)\right\| \leq\left\|S^{t}(h)-y_{k}\right\|+\left\|y_{k}-P_{V} S^{t}(h)\right\| \leq 2\left\|S^{t}(h)-y_{k}\right\|<\frac{\varepsilon}{2} .
\end{gathered}
$$

Assim,

$$
\begin{gathered}
\left\|P_{V} S-S\right\|=\sup _{h \in B_{H}}\left\|P_{V} S(h)-S(h)\right\|<\frac{\varepsilon}{2} \text { e } \\
\left\|P_{V} S^{t}-S^{t}\right\|=\sup _{h \in B_{H}}\left\|P_{V} S^{t}(h)-S^{t}(h)\right\|<\frac{\varepsilon}{2} .
\end{gathered}
$$

Segue que

$$
\begin{aligned}
\left\|P_{V} S P_{V}-S\right\| & \leq\left\|P_{V} S P_{V}-P_{V} S\right\|+\left\|P_{V} S-S\right\| \leq\left\|P_{V}\right\|\left\|S P_{V}-S\right\|+\left\|P_{V} S-S\right\| \\
& \leq\left\|P_{V} S^{t}-S^{t}\right\|+\left\|P_{V} S-S\right\|<\frac{\varepsilon}{2}+\frac{\varepsilon}{2}=\varepsilon .
\end{aligned}
$$

Veremos, na próxima proposição, que todo espaço de Hilbert satisfaz a uma equação análoga à equação da definição da propriedade $\mathcal{P}$.

Proposição 3.2.4. Sejam $H$ um espaço de Hilbert, $V$ e $W$ subespaços fechados de $H$. Consideremos $P_{V}$ e $Q_{W}$ as projeções ortogonais sobre $V$ e $W$ respectivamente, e $I$ o operador identidade em $H$. Então, para todos os operadores $T, S \in L(H)$, temos que

$$
\left\|Q_{W} T P_{V}+\left(I-Q_{W}\right) S\left(I-P_{V}\right)\right\|=\max \left\{\left\|Q_{W} T P_{V}\right\|,\left\|\left(I-Q_{W}\right) S\left(I-P_{V}\right)\right\|\right\} .
$$

Demonstração. Sejam $H$ um espaço de Hilbert, $V$ e $W$ subespaços fechados de $H$ e $T, S \in L(H)$. Consideremos

$$
\begin{array}{r}
Q_{W} T P_{V} \uparrow_{V}: V \longrightarrow W \quad \mathrm{e} \\
\left(I-Q_{W}\right) S\left(I-P_{V}\right)\left\lceil_{V^{\perp}}: V^{\perp} \longrightarrow W^{\perp}\right.
\end{array}
$$

Assim,

$\| Q_{W} T P_{V} \uparrow_{V} \oplus\left(I-Q_{W}\right) S\left(I-P_{V}\right)\left\lceil_{V^{\perp}} \|=\max \left\{\left\|Q_{W} T P_{V} \uparrow_{V}\right\|, \|\left(I-Q_{W}\right) S\left(I-P_{V}\right)\left\lceil_{V^{\perp}} \|\right\}\right.\right.$.

Mostraremos que $\left\|Q_{W} T P_{V} \uparrow_{V}\right\|=\left\|Q_{W} T P_{V}\right\|$. Para todo $h \in B_{H}$, temos que $h=x_{1}+x_{2}$, sendo $x_{1} \in V$ e $x_{2} \in V^{\perp}$. Como $\|h\|^{2}=\left\|x_{1}\right\|^{2}+\left\|x_{2}\right\|^{2}$, segue que $\left\|x_{1}\right\| \leq 1$. Então,

$$
\begin{aligned}
\left\|Q_{W} T P_{V} \uparrow_{V}\right\| & =\sup _{v \in B_{V}}\left\|Q_{W} T P_{V}(v)\right\|=\sup _{v \in B_{V}}\left\|Q_{W} T(v)\right\| \\
& =\sup _{x_{1} \in B_{V}}\left\|Q_{W} T\left(x_{1}\right)\right\|=\sup _{h \in B_{H}}\left\|Q_{W} T P_{V}(h)\right\|=\left\|Q_{W} T P_{V}\right\| .
\end{aligned}
$$


Analogamente, $\left\|\left.\left(I-Q_{W}\right) S\left(I-P_{V}\right)\right|_{V^{\perp}}\right\|=\left\|\left(I-Q_{W}\right) S\left(I-P_{V}\right)\right\|$.

Além disso, $V \oplus V^{\perp}=H, W \oplus W^{\perp}=H$ e $Q_{W} T P_{V} \uparrow_{V} \oplus\left(I-Q_{W}\right) S\left(I-P_{V}\right) \uparrow_{V^{\perp}}$ como operador de $H$ em $H$ é igual ao operador $Q_{W} T P_{V}+\left(I-Q_{W}\right) S\left(I-P_{V}\right)$. Logo,

$$
\left\|Q_{W} T P_{V}+\left(I-Q_{W}\right) S\left(I-P_{V}\right)\right\|=\max \left\{\left\|Q_{W} T P_{V}\right\|,\left\|\left(I-Q_{W}\right) S\left(I-P_{V}\right)\right\|\right\} .
$$

Podemos obter uma equação para somas finitas de operadores análoga à equação da Proposição 3.2.4. Tal resultado será estudado nesta proposição:

Proposição 3.2.5. Seja $H$ espaço de Hilbert. Para cada $n \in \mathbb{N}$ fixado, sejam $V_{k}$ e $W_{k}$ subespaços de $H$ tais que $V_{k} \subset V_{k+1}$ e $W_{k} \subset W_{k+1}$, para todo $1 \leq k \leq n$. Consideremos $P_{V_{k}}$ e $Q_{W_{k}}$ as projeções canônicas sobre $V_{k}$ e $W_{k}$ respectivamente, $P_{V_{0}}=0$ e $Q_{W_{0}}=0$. Então, para toda sequência $\left(T_{k}\right) \subset L(H)$, temos que

$$
\left\|\sum_{k=1}^{n}\left(Q_{W_{k}}-Q_{W_{k-1}}\right) T_{k}\left(P_{V_{k}}-P_{V_{k-1}}\right)\right\|=\max _{1 \leq k \leq n}\left\|\left(Q_{W_{k}}-Q_{W_{k-1}}\right) T_{k}\left(P_{V_{k}}-P_{V_{k-1}}\right)\right\| .
$$

Demonstração. Para todo $p \geq 1$ e todo $k \geq p+1$, sendo $p, k \in \mathbb{N}$, temos que $V_{p} \subset V_{k-1} \subset V_{k}$ e $W_{p} \subset W_{k-1} \subset W_{k}$. Então, $Q_{W_{p}} Q_{W_{k}}=Q_{W_{p}} Q_{W_{k-1}}=Q_{W_{p}}$ e $P_{V_{k}} P_{V_{p}}=P_{V_{k-1}} P_{V_{p}}=P_{V_{p}}$. Assim,

$$
\begin{aligned}
& \left(I-Q_{W_{p}}\right)\left(Q_{W_{k}}-Q_{W_{k-1}}\right) T_{k}\left(P_{V_{k}}-P_{V_{k-1}}\right)\left(I-P_{V_{p}}\right) \\
& =\left(Q_{W_{k}}-Q_{W_{k-1}}-Q_{W_{p}} Q_{W_{k}}+Q_{W_{p}} Q_{W_{k-1}}\right) T_{k}\left(P_{V_{k}}-P_{V_{k-1}}-P_{V_{k}} P_{V_{p}}+P_{V_{k-1}} P_{V_{p}}\right) \\
& =\left(Q_{W_{k}}-Q_{W_{k-1}}-Q_{W_{p}}+Q_{W_{p}}\right) T_{k}\left(P_{V_{k}}-P_{V_{k-1}}-P_{V_{p}}+P_{V_{p}}\right) \\
& =\left(Q_{W_{k}}-Q_{W_{k-1}}\right) T_{k}\left(P_{V_{k}}-P_{V_{k-1}}\right) .
\end{aligned}
$$

Faremos indução sobre $n$. Para $n=1,2$ é trivial. Para $n=3$, segue da Proposição 3.2.4 e da igualdade acima que

$$
\begin{aligned}
\| & \sum_{k=1}^{3}\left(Q_{W_{k}}-Q_{W_{k-1}}\right) T_{k}\left(P_{V_{k}}-P_{V_{k-1}}\right) \| \\
= & \left\|Q_{W_{1}} T_{1} P_{V_{1}}+\left(Q_{W_{2}}-Q_{W_{1}}\right) T_{2}\left(P_{V_{2}}-P_{V_{1}}\right)+\left(Q_{W_{3}}-Q_{W_{2}}\right) T_{3}\left(P_{V_{3}}-P_{V_{2}}\right)\right\| \\
= & \| Q_{W_{1}} T_{1} P_{V_{1}}+\left(I-Q_{W_{1}}\right)\left(Q_{W_{2}}-Q_{W_{1}}\right) T_{2}\left(P_{V_{2}}-P_{V_{1}}\right)\left(I-P_{V_{1}}\right) \\
& +\left(I-Q_{W_{1}}\right)\left(Q_{W_{3}}-Q_{W_{2}}\right) T_{3}\left(P_{V_{3}}-P_{V_{2}}\right)\left(I-P_{V_{1}}\right) \| \\
= & \left\|Q_{W_{1}} T_{1} P_{V_{1}}+\left(I-Q_{W_{1}}\right)\left[\left(Q_{W_{2}}-Q_{W_{1}}\right) T_{2}\left(P_{V_{2}}-P_{V_{1}}\right)+\left(Q_{W_{3}}-Q_{W_{2}}\right) T_{3}\left(P_{V_{3}}-P_{V_{2}}\right)\right]\left(I-P_{V_{1}}\right)\right\| \\
= & \max \left\{\left\|Q_{W_{1}} T_{1} P_{V_{1}}\right\|,\left\|\left(I-Q_{W_{1}}\right)\left[\left(Q_{W_{2}}-Q_{W_{1}}\right) T_{2}\left(P_{V_{2}}-P_{V_{1}}\right)+\left(Q_{W_{3}}-Q_{W_{2}}\right) T_{3}\left(P_{V_{3}}-P_{V_{2}}\right)\right]\left(I-P_{V_{1}}\right)\right\|\right\} \\
= & \max \left\{\left\|Q_{W_{1}} T_{1} P_{V_{1}}\right\|,\left\|Q_{W_{2}}\left(I-Q_{W_{1}}\right) T_{2}\left(I-P_{V_{1}}\right) P_{V_{2}}+\left(I-Q_{W_{2}}\right) Q_{W_{3}} T_{3} P_{V_{3}}\left(I-P_{V_{2}}\right)\right\|\right\} \\
= & \max \left\{\left\|Q_{W_{1}} T_{1} P_{V_{1}}\right\|, \max \left\{\left\|Q_{W_{2}}\left(I-Q_{W_{1}}\right) T_{2}\left(I-P_{V_{1}}\right) P_{V_{2}}\right\|,\left\|\left(I-Q_{W_{2}}\right) Q_{W_{3}} T_{3} P_{V_{3}}\left(I-P_{V_{2}}\right)\right\|\right\}\right\} \\
= & \max \left\{\left\|Q_{W_{1}} T_{1} P_{V_{1}}\right\|,\left\|Q_{W_{2}}\left(I-Q_{W_{1}}\right) T_{2}\left(I-P_{V_{1}}\right) P_{V_{2}}\right\|,\left\|\left(I-Q_{W_{2}}\right) Q_{W_{3}} T_{3} P_{V_{3}}\left(I-P_{V_{2}}\right)\right\|\right\} \\
= & \max _{1 \leq k \leq 3}\left\|\left(Q_{W_{k}}-Q_{W_{k-1}}\right) T_{k}\left(P_{V_{k}}-P_{V_{k-1}}\right)\right\| .
\end{aligned}
$$


Supondo que valha para $n$ :

$$
\left\|\sum_{k=1}^{n}\left(Q_{W_{k}}-Q_{W_{k-1}}\right) T_{k}\left(P_{V_{k}}-P_{V_{k-1}}\right)\right\|=\max _{1 \leq k \leq n}\left\|\left(Q_{W_{k}}-Q_{W_{k-1}}\right) T_{k}\left(P_{V_{k}}-P_{V_{k-1}}\right)\right\| .
$$

Mostraremos que vale para $n+1$.

$$
\begin{aligned}
& \left\|\sum_{k=1}^{n+1}\left(Q_{W_{k}}-Q_{W_{k-1}}\right) T_{k}\left(P_{V_{k}}-P_{V_{k-1}}\right)\right\| \\
& =\max \left\{\left\{\left\|\left(Q_{W_{k}}-Q_{W_{k-1}}\right) T_{k}\left(P_{V_{k}}-P_{V_{k-1}}\right)\right\|: 1 \leq k \leq n-1\right\},\right. \\
& \left.\left\|\left(Q_{W_{n}}-Q_{W_{n-1}}\right) T_{n}\left(P_{V_{n}}-P_{V_{n-1}}\right)+\left(Q_{W_{n+1}}-Q_{W_{n}}\right) T_{n+1}\left(P_{V_{n+1}}-P_{V_{n}}\right)\right\|\right\} \\
& =\max \left\{\left\{\left\|\left(Q_{W_{k}}-Q_{W_{k-1}}\right) T_{k}\left(P_{V_{k}}-P_{V_{k-1}}\right)\right\|: 1 \leq k \leq n-1\right\},\right. \\
& \left.\quad\left\|Q_{W_{n}}\left(I-Q_{W_{n-1}}\right) T_{n}\left(I-P_{V_{n-1}}\right) P_{V_{n}}+\left(I-Q_{W_{n}}\right) Q_{W_{n+1}} T_{n+1} P_{V_{n+1}}\left(I-P_{V_{n}}\right)\right\|\right\} \\
& =\max \left\{\left\{\left\|\left(Q_{W_{k}}-Q_{W_{k-1}}\right) T_{k}\left(P_{V_{k}}-P_{V_{k-1}}\right)\right\|: 1 \leq k \leq n-1\right\},\right. \\
& \left.\quad \max \left\{\left\|Q_{W_{n}}\left(I-Q_{W_{n-1}}\right) T_{n}\left(I-P_{V_{n-1}}\right) P_{V_{n}}\right\|,\left\|\left(I-Q_{W_{n}}\right) Q_{W_{n+1}} T_{n+1} P_{V_{n+1}}\left(I-P_{V_{n}}\right)\right\|\right\}\right\} \\
& =\max _{1 \leq k \leq n+1}\left\|\left(Q_{W_{k}}-Q_{W_{k-1}}\right) T_{k}\left(P_{V_{k}}-P_{V_{k-1}}\right)\right\| .
\end{aligned}
$$

Lema 3.2.6. Sejam $H$ espaço de Hilbert e $V$ um subespaço de $H$ de dimensão finita. Consideremos $P_{V}$ a projeção ortogonal de $H$ sobre $V$. Se, para todo $h \in H$, os operadores avaliação

$$
\begin{array}{rr}
\delta_{h}: M \longrightarrow H & \gamma_{h}: M \longrightarrow H \\
S \mapsto S(h) & S \mapsto S^{t}(h)
\end{array}
$$

são operadores DP1, então, para toda sequência $T_{n} \stackrel{w}{\longrightarrow} T$ em $M$, com $\left\|T_{n}\right\|=\|T\|=1$, temos que $\left\|T_{n}-\left(I-P_{V}\right) T_{n}\left(I-P_{V}\right)\right\| \longrightarrow 0$.

Demonstração. Como $P_{V}$ é um operador de posto finito, então é compacto. Pelo Teorema 1.3.8, temos que $P_{V}^{t}$ também é compacto. Segue, da Proposição 3.2.2, que os operadores

$$
\begin{array}{rlrl}
\delta_{P_{V}}: M & \longrightarrow K(H) & \gamma_{P_{V}^{t}}: M & \longrightarrow K(H) \\
S & \mapsto S P_{V} & S \mapsto S^{t} P_{V}^{t}
\end{array}
$$

são operadores DP1. Então,

$$
\begin{aligned}
\delta_{P_{V}}\left(T_{n}\right) \longrightarrow 0 & \Longrightarrow T_{n} P_{V} \longrightarrow 0 \Longrightarrow\left\|T_{n} P_{V}\right\| \longrightarrow 0 \mathrm{e} \\
\gamma_{P_{V}^{t}}\left(T_{n}\right) \longrightarrow 0 & \Longrightarrow T_{n}^{t} P_{V}^{t} \longrightarrow 0 \Longrightarrow\left\|T_{n}^{t} P_{V}^{t}\right\|=\left\|P_{V} T_{n}\right\| \longrightarrow 0 .
\end{aligned}
$$


Além disso, pelo Teorema 1.3.1, temos que $\left\|P_{V}\right\|=1$. Então,

$$
\begin{aligned}
\left\|T_{n}-\left(I-P_{V}\right) T_{n}\left(I-P_{V}\right)\right\| & =\left\|T_{n}-\left(T_{n}-P_{V} T_{n}\right)\left(I-P_{V}\right)\right\| \\
& =\left\|T_{n}-T_{n}+T_{n} P_{V}+P_{V} T_{n}\left(I-P_{V}\right)\right\| \\
& \leq\left\|T_{n} P_{V}\right\|+\left\|P_{V} T_{n}\right\|\left\|I-P_{V}\right\| \longrightarrow 0 .
\end{aligned}
$$

O seguinte teorema caracterizará subespaços fechados do espaço dos operadores compactos em espaços de Hilbert arbitrários (não necessariamente separáveis) satisfazendo à propriedade DP1, sendo este o principal resultado desta seção. A demonstração utilizará a mesma ideia da demonstração do Teorema 3.1.14 e os resultados em espaços de Hilbert que foram estudados ao longo desta seção.

Teorema 3.2.7. Sejam $H$ espaço de Hilbert e $M$ subespaço fechado de $K(H)$. Então, $M$ tem a propriedade DP1 se, e somente se, para todo $h \in H$, os operadores avaliação

$$
\begin{array}{rlrl}
\delta_{h}: M \longrightarrow H & \gamma_{h}: M \longrightarrow H \\
S & \mapsto S(h) & S & \mapsto S^{t}(h)
\end{array}
$$

são operadores DP1.

Demonstração. $\Rightarrow$ Segue da Proposição 3.2.1.

$\Leftarrow$ Suponhamos que $M$ não tem a propriedade DP1. Então, existem $T_{n} \stackrel{w}{\longrightarrow} 0$ em $M, T \in M$, com $\left\|T_{n}+T\right\|=\|T\|=1$, e $f_{n} \stackrel{w}{\longrightarrow} 0$ em $M^{*}$ tais que $f_{n}\left(T_{n}\right) \nrightarrow 0$, isto é, existe $r>0$ tal que, para todo $n \in \mathbb{N}$,

$$
\left|f_{n}\left(T_{n}\right)\right| \geq r .
$$

Construíremos uma subsequência de $\left(f_{n}\right)_{n}$ que não converge fracamente a zero. Isso nos levará a uma contradição, pois $\left(f_{n}\right)_{n}$ converge fracamente a zero.

Consideremos $f_{\sigma(1)}=f_{1}, T_{\sigma(1)}=T_{1}$. Como $T_{\sigma(1)} \in K(X, Y)$, pela Proposição 3.2.3, existe $V_{1}$ subespaço de $H$ de dimensão finita tal que

$$
\left\|T_{\sigma(1)}-P_{V_{1}} T_{\sigma(1)} P_{V_{1}}\right\|<\frac{1}{2}
$$

em que $P_{V_{1}}$ é a projeção de $H$ sobre $V_{1}$.

Segue, pelo Lema 3.2.6, que para um $n$ suficientemente grande,

$$
\left\|T_{n}-\left(I-P_{V_{1}}\right) T_{n}\left(I-P_{V_{1}}\right)\right\|<\frac{1}{2^{4}} .
$$

Como $T_{n} \stackrel{w}{\longrightarrow} 0$ e $f_{n} \stackrel{w}{\longrightarrow} 0$, temos que

$$
f_{\sigma_{1}}\left(T_{n}\right) \longrightarrow 0 \Longrightarrow\left|f_{\sigma_{1}}\left(T_{n}\right)\right|<\frac{r}{2^{3}} \quad \text { e } \quad f_{n}\left(T_{\sigma_{1}}\right) \longrightarrow 0 \Longrightarrow\left|f_{n}\left(T_{\sigma_{1}}\right)\right|<\frac{r}{2^{3}}
$$


Escolhemos $\sigma(2)<\sigma(1)$ satisfazendo a essas condições, isto é,

$$
\begin{array}{r}
\left\|T_{\sigma(2)}-\left(I-P_{V_{1}}\right) T_{\sigma(2)}\left(I-P_{V_{1}}\right)\right\|<\frac{1}{2^{4}}, \\
\left|f_{\sigma_{1}}\left(T_{n}\right)\right|<\frac{r}{2^{3}}, \\
\left|f_{n}\left(T_{\sigma_{1}}\right)\right|<\frac{r}{2^{3}} \quad \mathrm{e} \\
\left|f_{\sigma(2)\left(T_{\sigma(2)}\right)}\right| \geq r .
\end{array}
$$

Como $T_{\sigma(2)}$ é compacto, então $\left(I-P_{V_{1}}\right) T_{\sigma(2)}\left(I-P_{V_{1}}\right)$ é um operador compacto. Pela Proposição 3.2.3, existe $V_{2} \supset V_{1}$ subespaço de $H$ de dimensão finita tal que

$$
\left\|\left(I-P_{V_{1}}\right) T_{\sigma(2)}\left(I-P_{V_{1}}\right)-P_{V_{2}}\left(I-P_{V_{1}}\right) T_{\sigma(2)}\left(I-P_{V_{1}}\right) P_{V_{2}}\right\|<\frac{1}{2^{4}},
$$

em que $P_{V_{2}}$ é a projeção ortogonal de $H$ sobre $V_{2}$.

Observemos que $V_{2} \supset V_{1}$, então $P_{V_{2}} P_{V_{1}}=P_{V_{1}} P_{V_{2}}=P_{V_{1}}$, donde $P_{V_{2}}\left(I-P_{V_{1}}\right) T_{\sigma(2)}\left(I-P_{V_{1}}\right) P_{V_{2}}=$ $\left(P_{V_{2}}-P_{V_{2}} P_{V_{1}}\right) T_{\sigma(2)}\left(P_{V_{2}}-P_{V_{1}} P_{V_{2}}\right)=\left(P_{V_{2}}-P_{V_{1}}\right) T_{\sigma(2)}\left(P_{V_{2}}-P_{V_{1}}\right)$. Assim:

$$
\begin{aligned}
& \left\|T_{\sigma(2)}-\left(P_{V_{2}}-P_{V_{1}}\right) T_{\sigma(2)}\left(P_{V_{2}}-P_{V_{1}}\right)\right\| \\
= & \left\|T_{\sigma(2)}-\left(I-P_{V_{1}}\right) T_{\sigma(2)}\left(I-P_{V_{1}}\right)+\left(I-P_{V_{1}}\right) T_{\sigma(2)}\left(I-P_{V_{1}}\right)-\left(P_{V_{2}}-P_{V_{1}}\right) T_{\sigma(2)}\left(P_{V_{2}}-P_{V_{1}}\right)\right\| \\
\leq & \left\|T_{\sigma(2)}-\left(I-P_{V_{1}}\right) T_{\sigma(2)}\left(I-P_{V_{1}}\right)\right\|+\left\|\left(I-P_{V_{1}}\right) T_{\sigma(2)}\left(I-P_{V_{1}}\right)-\left(P_{V_{2}}-P_{V_{1}}\right) T_{\sigma(2)}\left(P_{V_{2}}-P_{V_{1}}\right)\right\| \\
< & \frac{1}{2^{4}}+\frac{1}{2^{4}}=\frac{1}{2^{3}} .
\end{aligned}
$$

Suponhamos que escolhemos $V_{1} \subset V_{2} \subset \cdots \subset V_{n}$ subespaços de $H$ de dimensão finita e os $n$ primeiros índices da subsequência, denotados por $\sigma(1)<\sigma(2)<\cdots<\sigma(n) \in \mathbb{N}$, tais que

$$
\begin{aligned}
& \left\|T_{\sigma(i)}-\left(P_{V_{i}}-P_{V_{i-1}}\right) T_{\sigma(i)}\left(P_{V_{i}}-P_{V_{i-1}}\right)\right\|<\frac{1}{2^{i+1}}, \\
& \left|f_{\sigma(i)}\left(T_{\sigma(i)}\right)\right| \geq r, \quad 1 \leq i \leq n, \\
& \left|f_{\sigma(i)}\left(T_{\sigma(n)}\right)\right|<\frac{r}{2^{n+1}} \quad \mathrm{e} \\
& \left|f_{\sigma(n)}\left(T_{\sigma(i)}\right)\right|<\frac{r}{2^{n+1}}, \quad i<n .
\end{aligned}
$$

Pelo Lema 3.2.6, para um $p$ suficientemente grande, temos que

$$
\left\|T_{p}-\left(I-P_{V_{n}}\right) T_{p}\left(I-P_{V_{n}}\right)\right\|<\frac{1}{2^{n+2}} .
$$

Como $T_{p} \stackrel{w}{\longrightarrow} 0$ e $f_{p} \stackrel{w}{\longrightarrow} 0$, para todo $i \leq p$, temos que

$$
f_{\sigma_{i}}\left(T_{p}\right) \longrightarrow 0 \Longrightarrow\left|f_{\sigma_{i}}\left(T_{p}\right)\right|<\frac{r}{2^{n+2}} \quad \text { e } \quad f_{p}\left(T_{\sigma_{i}}\right) \longrightarrow 0 \Longrightarrow\left|f_{p}\left(T_{\sigma_{i}}\right)\right|<\frac{r}{2^{n+2}} .
$$


Escolhemos $\sigma(n+1)>\sigma(n)$ satisfazendo às condições anteriores, isto é,

$$
\begin{gathered}
\left\|T_{\sigma(n+1)}-\left(I-P_{V_{n}}\right) T_{\sigma(n+1)}\left(I-P_{V_{n}}\right)\right\|<\frac{1}{2^{n+2}}, \\
\left|f_{\sigma_{1}}\left(T_{\sigma(n+1)}\right)\right|<\frac{r}{2^{n+2}}, \\
\left|f_{\sigma(i)}\left(T_{\sigma_{i}}\right)\right|<\frac{r}{2^{n+2}} \quad \mathrm{e} \\
\left|f_{\sigma(n+1)}\left(T_{\sigma(2)}\right)\right| \geq r, \quad i \leq n .
\end{gathered}
$$

Pela Proposição 3.2.3, podemos aproximar o operador compacto $\left(I-P_{V_{n}}\right) T_{\sigma(n+1)}\left(I-P_{V_{n}}\right)$, isto é, existe $V_{n+1} \supset V_{n}$ subespaço de $H$ de dimensão finita tal que

$$
\left\|\left(I-P_{V_{n}}\right) T_{\sigma(n+1)}\left(I-P_{V_{n}}\right)-P_{V_{n+1}}\left(I-P_{V_{n}}\right) T_{\sigma(n+1)}\left(I-P_{V_{n}}\right) P_{V_{n+1}}\right\|<\frac{1}{2^{n+1}},
$$

em que $P_{V_{n+1}}$ é a projeção ortogonal de $H$ sobre $V$.

Observemos novamente que $P_{V_{n+1}} P_{V_{n}}=P_{V_{n}} P_{V_{n+1}}=P_{V_{n}}$. Assim:

$$
\begin{aligned}
& \left\|T_{\sigma(n+1)}-\left(P_{V_{n+1}}-P_{V_{n}}\right) T_{\sigma(n+1)}\left(P_{V_{n+1}}-P_{V_{n}}\right)\right\| \\
\leq & \left\|T_{\sigma(n+1)}-\left(I-P_{v_{n}}\right) T_{\sigma(n+1)}\left(I-P_{V_{n}}\right)\right\| \\
+ & \left\|\left(I-P_{V_{n}}\right) T_{\sigma(n+1)}\left(I-P_{V_{n}}\right)-\left(P_{V_{n+1}}-P_{V_{n}}\right) T_{\sigma(n+1)}\left(P_{V_{n+1}}-P_{V_{n}}\right)\right\| \\
< & \frac{1}{2^{n+3}}+\frac{1}{2^{n+3}}=\frac{1}{2^{n+2}} .
\end{aligned}
$$

Além disso,

$$
\begin{aligned}
\left|f_{\sigma(n+1)}\left(T_{\sigma(n+1)}\right)\right| \geq r & \\
\left|f_{\sigma(n+1)}\left(T_{\sigma(i)}\right)\right|<\frac{r}{2^{n+1}} \quad \text { e } & \\
\left|f_{\sigma(i)}\left(T_{\sigma(n+1)}\right)\right|<\frac{r}{2^{n+1}}, & i<n+1 .
\end{aligned}
$$

Dessa forma, construímos $\left(f_{\sigma(n)}\right)_{n}$ uma subsequência de $\left(f_{n}\right)_{n}$. Mostraremos que $f_{\sigma(n)} \stackrel{w}{\rightarrow} 0$.

Primeiramente, vejamos que $S_{n}=\sum_{k=1}^{n} T_{\sigma(k)}$ é limitado. Consideremos $P_{V_{0}}=0$. Pela Proposição 3.2 .5 , segue que

$$
\begin{aligned}
& \left\|\sum_{k=1}^{n}\left(P_{V_{k}}-P_{V_{k-1}}\right) T_{\sigma(k)}\left(P_{V_{k}}-P_{V_{k-1}}\right)\right\|=\max _{1 \leq k \leq n}\left\|\left(P_{V_{k}}-P_{V_{k-1}}\right) T_{\sigma(k)}\left(P_{V_{k}}-P_{V_{k-1}}\right)\right\| \\
& \leq\left(\max _{1 \leq k \leq n}\left\|P_{V_{k}}\right\|+\max _{1 \leq k \leq n}\left\|P_{V_{k-1}}\right\|\right) \max _{1 \leq k \leq n}\left\|T_{\sigma(k)}\right\|\left(\max _{1 \leq k \leq n}\left\|P_{V_{k}}\right\|+\max _{1 \leq k \leq n}\left\|P_{V_{k-1}}\right\|\right) \\
& \leq 4 \max _{1 \leq k \leq n}\left\|T_{\sigma(k)}\right\| .
\end{aligned}
$$

Como $T_{\sigma(k)} \stackrel{w}{\longrightarrow} 0$ em $M$, então $\left(T_{\sigma(k)}\right)_{n}$ é limitada, isto é, existe $D>0$ tal que $\left\|T_{\sigma(k)}\right\| \leq D$, para todo $n \in \mathbb{N}$. 
Temos ainda que

$$
\begin{aligned}
\left\|\sum_{k=1}^{n} T_{\sigma(k)}\right\| & \leq\left\|\sum_{k=1}^{n} T_{\sigma(k)}-\sum_{k=1}^{n}\left(P_{V_{k}}-P_{V_{k-1}}\right) T_{\sigma(k)}\left(P_{V_{k}}-P_{V_{k-1}}\right)\right\|+\left\|\sum_{k=1}^{n}\left(P_{V_{k}}-P_{V_{k-1}}\right) T_{\sigma(k)}\left(P_{V_{k}}-P_{V_{k-1}}\right)\right\| \\
& \leq \sum_{k=1}^{n}\left\|T_{\sigma(k)}-\sum_{k=1}^{n}\left(P_{V_{k}}-P_{V_{k-1}}\right) T_{\sigma(k)}\left(P_{V_{k}}-P_{V_{k-1}}\right)\right\|+\left\|\sum_{k=1}^{n}\left(P_{V_{k}}-P_{V_{k-1}}\right) T_{\sigma(k)}\left(P_{V_{k}}-P_{V_{k-1}}\right)\right\| \\
& \leq \sum_{k=1}^{n} \frac{1}{2^{k}}+4 D .
\end{aligned}
$$

Assim, $\left(S_{n}\right)_{n}$ é uma sequência limitada em $M$. Pela Proposição 3.1.6, existe $\varphi \in M^{* *}$ um ponto de acumulação fraco-estrela de $\left(S_{m}\right)$, isto é, $C\left(S_{m}\right) \stackrel{w^{*}}{\longrightarrow} \varphi$ em $M^{* *}$. Então

$$
\varphi_{m}=C\left(S_{m}\right) \stackrel{w^{*}}{\longrightarrow} \varphi \text { em } M^{* *} \Longleftrightarrow C\left(S_{m}\right)(f) \longrightarrow \varphi(f), \quad \forall f \in M^{*} .
$$

Fixemos $n \in \mathbb{N}$,

$$
C\left(S_{m}\right)\left(f_{\sigma(n)}\right) \longrightarrow \varphi\left(f_{\sigma(n)}\right) \Rightarrow f_{\sigma(n)}\left(S_{m}\right) \longrightarrow \varphi\left(f_{\sigma(n)}\right)
$$

Para $p>n$ e usando (3.3) e (3.4), temos que

$$
\begin{aligned}
\left|f_{\sigma(n)}\left(\sum_{k=1}^{p} T_{\sigma(k)}\right)\right| & =\left|\sum_{k=1}^{p} f_{\sigma(n)}\left(T_{\sigma(k)}\right)\right|=\left|f_{\sigma(n)}\left(T_{\sigma(n)}\right)+\sum_{k=1_{k \neq n}}^{p} f_{\sigma(n)}\left(T_{\sigma(k)}\right)\right| \\
& \geq\left|f_{\sigma(n)}\left(T_{\sigma(n)}\right)\right|-\left|\sum_{k=1_{k \neq n}}^{p} f_{\sigma(n)}\left(T_{\sigma(k)}\right)\right| \geq\left|f_{\sigma(n)}\left(T_{\sigma(n)}\right)\right|-\sum_{k=1_{k \neq n}}^{p}\left|f_{\sigma(n)}\left(T_{\sigma(k)}\right)\right| \\
& \geq r-\sum_{k=1}^{n-1}\left|f_{\sigma(n)}\left(T_{\sigma(k)}\right)\right|-\sum_{k=n+1}^{p}\left|f_{\sigma(n)}\left(T_{\sigma(k)}\right)\right| \geq r-\sum_{k=1}^{n-1} \frac{r}{2^{n+1}}-\sum_{k=n+1}^{p} \frac{r}{2^{k+1}} \\
& =r\left(1-\left(\sum_{k=1}^{n-1} \frac{1}{2^{n+1}}-\sum_{k=n+1}^{p} \frac{1}{2^{k+1}}\right)\right) \geq r\left(1-\sum_{k=1}^{p} \frac{1}{2^{k+1}}\right) \\
& \geq r\left(1-\sum_{k=1}^{\infty} \frac{1}{2^{k+1}}\right)=r\left(1-\frac{1}{2}\right)=\frac{r}{2} .
\end{aligned}
$$

Assim,

$$
\left|f_{\sigma(n)}\left(S_{m_{k}}\right)-\varphi\left(f_{\sigma(n)}\right)\right| \geq\left|f_{\sigma(n)}\left(S_{m_{k}}\right)\right|-\left|\varphi\left(f_{\sigma(n)}\right)\right| \geq \frac{r}{2}-\left|\varphi\left(f_{\sigma(n)}\right)\right| .
$$

Então, para todo $n \in \mathbb{N}$, temos que

$$
\frac{r}{2}-\left|\varphi\left(f_{\sigma(n)}\right)\right| \leq\left|f_{\sigma(n)}\left(S_{m_{k}}\right)-\varphi\left(f_{\sigma(n)}\right)\right| \longrightarrow 0 \Longrightarrow\left|\varphi\left(f_{\sigma(n)}\right)\right| \geq \frac{r}{2}, \quad \forall n \in \mathbb{N} .
$$

Logo, $\varphi\left(f_{\sigma(n)}\right) \nrightarrow 0$ e, portanto, $f_{\sigma(n)} \stackrel{w}{\nrightarrow} 0$.

Como $\left(f_{n}\right)_{n}$ converge fracamente a zero, temos uma contradição. Logo, $M$ tem a propriedade DP1. 
Veremos a seguir, a partir de um exemplo, que a hipótese do subespaço $M$ ser um subespaço do espaço dos operadores compactos no Teorema 3.2.7 é essencial.

O exemplo a seguir nos mostra que a hipótese do subespaço $M$ ser um subespaço dos operadores compactos no Teorema 3.2.7 é essencial.

Exemplo 3.2.8. Sejam $H=\ell_{2}$ e $V_{n}, W_{n}$ subespaços de $H$ dois a dois ortogonais tais que $H$ pode se decompor na soma direta

$$
\ell_{2}=\left(\oplus_{n} V_{n}\right)_{2} \oplus_{2}\left(\oplus_{n} W_{n}\right)_{2}
$$

e $\operatorname{dim}\left(V_{n}\right)=\operatorname{dim}\left(W_{n}\right)=n$.

Por exemplo, tomemos $V_{i}=\left[e_{2 k-1}: k \leq i\right]$ e $W_{i}=\left[e_{2 k}: k \leq i\right]$.

Seja

$$
M=\left(\oplus_{n=1}^{\infty} L\left(V_{n}\right)\right)_{\infty} \oplus_{\infty}\left(\oplus_{n=1}^{\infty} L\left(W_{n}\right)\right)_{\infty}
$$

Vejamos que $M \subset L\left(\ell_{2}\right)$. Sejam $T_{n}: V_{n} \longrightarrow V_{n}$ e $S_{n}: W_{n} \longrightarrow W_{n}$. Então, $\left(\oplus_{n=1}^{\infty} T_{n}\right)_{\infty} \oplus_{\infty}$ $\left(\oplus_{n=1}^{\infty} S_{n}\right) \in M$. Mas,

$$
\left(\oplus_{n=1}^{\infty} T_{n}\right)_{\infty} \oplus_{\infty}\left(\oplus_{n=1}^{\infty} S_{n}\right)_{\infty}:\left(\oplus_{n} V_{n}\right)_{2} \oplus_{2}\left(\oplus_{n} W_{n}\right)_{2} \longrightarrow\left(\oplus_{n} V_{n}\right)_{2} \oplus_{2}\left(\oplus_{n} W_{n}\right)_{2}
$$

Assim, $\left(\oplus_{n=1}^{\infty} T_{n}\right)_{\infty} \oplus_{\infty}\left(\oplus_{n=1}^{\infty} S_{n}\right) \in L\left(\ell_{2}\right)$.

Vamos mostrar que $M$ não tem a propriedade DP1. Primeiramente, vejamos que $\left(\oplus_{k=1}^{\infty} L\left(V_{n_{k}}\right)\right)_{\infty}$ contém uma cópia complementada isométrica de $\left(\oplus_{k=1}^{\infty} l_{2}^{n_{k}}\right)_{\infty}$. Consideremos

$$
\begin{gathered}
\left(\oplus_{k=1}^{\infty} M_{n_{k}}(\mathbb{C})\right)_{\infty}=\left\{A=\left(\begin{array}{ccccc}
A_{1} & 0 & \cdots & 0 & \cdots \\
0 & A_{2} & \cdots & 0 & \cdots \\
\vdots & \vdots & \ddots & \vdots & \vdots \\
0 & 0 & \cdots & A_{k} & \cdots \\
\vdots & \vdots & \vdots & \vdots & \vdots
\end{array}\right): A_{k} \in M_{n_{k}}(\mathbb{C}), \sup _{k \in \mathbb{N}}\left\|A_{k}\right\|<\infty\right\} \\
\|A\|=\sup _{k \in \mathbb{N}}\left\|A_{k}\right\|=\sup _{k \in \mathbb{N}}\left(\sum_{i, j}^{n_{k}}\left|a_{i j}\right|^{2}\right)^{\frac{1}{2}}
\end{gathered}
$$


Definimos $T:\left(\oplus_{k=1}^{\infty} l_{2}^{n_{k}}\right)_{\infty} \longrightarrow\left(\oplus_{k=1}^{\infty} M_{n_{k}}(\mathbb{C})\right)_{\infty}$ dado por

$$
\begin{aligned}
& T(x)=T\left(\left(x_{1}^{\left(n_{1}\right)},\left(x_{1}^{\left(n_{2}\right)}, x_{2}^{\left(n_{2}\right)}\right), \ldots,\left(x_{1}^{\left(n_{k}\right)}, \ldots, x_{n_{k}}^{\left(n_{k}\right)}\right), \ldots\right)\right) \\
& =\left(\begin{array}{ccccccc}
\left(x_{1}^{\left(n_{1}\right)}\right) & \mathbf{0} & \cdots & & \mathbf{0} & & \cdots \\
\mathbf{0} & \left(\begin{array}{ccc}
x_{1}^{\left(n_{2}\right)} & 0 \\
x_{2}^{\left(n_{2}\right)} & 0
\end{array}\right) & \cdots & & \mathbf{0} & & \cdots \\
\vdots & \vdots & \ddots & & \vdots & & \vdots \\
& & & & & & \\
\mathbf{0} & \mathbf{0} & \cdots & \left(\begin{array}{cccc}
x_{1}^{\left(n_{k}\right)} & 0 & \cdots & 0 \\
\vdots & \vdots & \ddots & \vdots \\
x_{n_{k}}^{\left(n_{k}\right)} & 0 & \cdots & 0
\end{array}\right) & \cdots \\
\vdots & \vdots & \vdots & & \vdots & & \vdots
\end{array}\right)
\end{aligned}
$$

Temos que $T$ está bem definido, pois, como $x \in\left(\oplus_{k=1}^{\infty} l_{2}^{n_{k}}\right)_{\infty}$, segue que $\sup _{k \in \mathbb{N}}\left(\sum_{i, j}^{n_{k}}\left|a_{i j}\right|^{2}\right)^{\frac{1}{2}}<\infty$, isto é, $T(x) \in\left(\oplus_{k=1}^{\infty} M_{n_{k}}(\mathbb{C})\right)_{\infty}$. Além disso, $T$ é uma isometria, pois

$$
\|T(x)\|=\sup _{k \in \mathbb{N}}\left(\sum_{i, j}^{n_{k}}\left|a_{i j}\right|^{2}\right)^{\frac{1}{2}}=\|x\| .
$$

Assim, $T$ é injetor e, pelo Teorema da Aplicação Aberta (Teorema 1.1.2), $T^{-1}: \operatorname{Im} T \longrightarrow$ $\left(\oplus_{k=1}^{\infty} l_{2}^{n_{k}}\right)_{\infty}$ é contínuo. Vejamos que $\operatorname{Im} T$ é um subespaço complementado de $\left(\oplus_{k=1}^{\infty} M_{n_{k}}(\mathbb{C})\right)_{\infty}$. Definimos

$$
\begin{gathered}
\pi:\left(\oplus_{k=1}^{\infty} M_{n_{k}}(\mathbb{C})\right)_{\infty} \longrightarrow \operatorname{Im} T \\
\left(\begin{array}{ccccc}
A_{1} & 0 & \cdots & 0 & \cdots \\
0 & A_{2} & \cdots & 0 & \cdots \\
\vdots & \vdots & \ddots & \vdots & \vdots \\
0 & 0 & \cdots & A_{k} & \cdots \\
\vdots & \vdots & \vdots & \vdots & \vdots
\end{array}\right) \longmapsto\left(\begin{array}{ccccc}
B_{1} & 0 & \cdots & 0 & \cdots \\
0 & B_{2} & \cdots & 0 & \cdots \\
\vdots & \vdots & \ddots & \vdots & \vdots \\
0 & 0 & \cdots & B_{k} & \cdots \\
\vdots & \vdots & \vdots & \vdots & \vdots
\end{array}\right)
\end{gathered}
$$

sendo

$$
B_{k}=\left(\begin{array}{cccc}
a_{11}^{(k)} & 0 & \cdots & 0 \\
a_{22}^{(k)} & 0 & \cdots & 0 \\
\vdots & \vdots & \ddots & \vdots \\
a_{n_{k} n_{k}}^{(k)} & 0 & \cdots & 0
\end{array}\right)
$$

Temos que $\pi$ está bem definido, é sobrejetor e $\pi^{2}=\pi$. Assim, $\left(\oplus_{k=1}^{\infty} L\left(V_{n_{k}}\right)\right)_{\infty}$ contém uma cópia complementada isométrica de $\left(\oplus_{k=1}^{\infty} l_{2}^{n_{k}}\right)_{\infty}$.

Além disso, vimos em 2.2.24 que $\left(\oplus_{k=1}^{\infty} l_{2}^{n_{k}}\right)_{\infty}$ contém uma cópia complementada isométrica de $\ell_{2}$. Então, $M$ contém uma cópia complementada isométrica de $\ell_{2} \oplus_{\infty} \ell_{2}$. Pelo Exemplo 2.2.14, $\ell_{2} \oplus_{\infty} \ell_{2}$ não tem a propriedade DP1, então $M$ não tem a propriedade DP1. 
Mostraremos que, para todo $h \in H$, os operadores avaliação

$$
\begin{aligned}
\delta_{h}: M & \longrightarrow H & \gamma_{h}: M & \longrightarrow H \\
m & \longmapsto m(h) & m & \longmapsto m^{t}(h)
\end{aligned}
$$

são operadores DP1.

Sejam $h \in H, m_{k} \stackrel{w}{\longrightarrow} 0$ em $M, m \in M$, com $\left\|m_{k}+m\right\|=\|m\|=1$. Vejamos que $\delta_{h}\left(m_{k}\right) \longrightarrow 0$.

Como $m_{k} \stackrel{w}{\longrightarrow} 0$, segue, pela Proposição 1.1.34, que existe $\alpha>0$ tal que $\left\|m_{k}\right\| \leq \alpha$, para todo $k \in \mathbb{N}$.

Dado $\varepsilon>0$, existem $p \in \mathbb{N}, v_{i} \in V_{i}$ e $w_{i} \in W_{i}$, para todo $i \leq p$, tal que

$$
\left\|h-\sum_{i=1}^{p}\left(v_{i}+w_{i}\right)\right\| \leq \frac{\varepsilon}{3 \alpha} .
$$

Assim,

$$
\begin{aligned}
\left\|\delta_{h}-\sum_{i=1}^{p}\left(\delta_{v_{i}}+\delta_{w_{i}}\right)\right\| & =\sup _{a \in B_{M}}\left\|\left(\delta_{h}-\sum_{i=1}^{p}\left(\delta_{v_{i}}+\delta_{w_{i}}\right)\right)(a)\right\| \\
& =\sup _{a \in B_{M}}\left\|a(h)-\sum_{i=1}^{p}\left(a\left(v_{i}\right)+a\left(w_{i}\right)\right)\right\| \\
& \leq \sup _{a \in B_{M}}\|a\|\left\|h-\sum_{i=1}^{p}\left(v_{i}+w_{i}\right)\right\| \leq \frac{\varepsilon}{3 \alpha} .
\end{aligned}
$$

Para todo $k \in \mathbb{N}$, temos que $m_{k} \in M$. Então, $m_{k}=\left(\oplus_{i=1}^{\infty} T_{i}\right)_{\infty} \oplus_{\infty}\left(\oplus_{i=1}^{\infty} S_{i}\right)_{\infty}$.

Consideremos $P_{V_{i}}$ a projeção de $H$ sobre $V_{i}$. Então, $m_{k}\left(v_{i}\right)=T_{i}\left(v_{i}\right)$ e $P_{V_{i}} m_{k} P_{V_{i}}\left(v_{i}\right)=P_{V_{i}} m_{k}\left(v_{i}\right)=$ $P_{V_{i}} T_{i}\left(v_{i}\right)=T_{i}\left(v_{i}\right)$. Assim, $m_{k}\left(v_{i}\right)=P_{V_{i}} m_{k} P_{V_{i}}\left(v_{i}\right)$, isto é, $m_{k} \uparrow_{V_{i}}=P_{V_{i}} m_{k} P_{V_{i}} \uparrow_{V_{i}}$, para todo $k \in \mathbb{N}$.

Como $\left(P_{V_{i}} m_{k} P_{V_{i}}\right)_{k}$ é uma sequência $w$-convegente a zero no espaço de dimensão finita $L\left(V_{i}\right)$ e $P_{V_{i}} m_{k} P_{V_{i}}\left\lceil\bigvee_{i} \stackrel{w}{\longrightarrow} 0\right.$ então $P_{V_{i}} m_{k} P_{V_{i}} \uparrow_{V_{i}} \longrightarrow 0$. Para todo $i \leq p$, temos que

$$
P_{V_{i}} m_{k} P_{V_{i}}\left\lceil_{V_{i}} \longrightarrow 0 \Longrightarrow P_{V_{i}} m_{k} P_{V_{i}}\left(v_{i}\right) \longrightarrow 0 \Longrightarrow m_{k}\left(v_{i}\right) \longrightarrow 0 \Longrightarrow \delta_{v_{i}}\left(m_{k}\right) \longrightarrow 0 .\right.
$$

Analogamente, considere $P_{W_{i}}$ a projeção de $H$ sobre $W_{i}$. Então, $m_{k}\left(w_{i}\right)=T_{i}\left(w_{i}\right)$ e $P_{W_{i}} m_{k} P_{W_{i}}\left(w_{i}\right)=$ $P_{W_{i}} m_{k}\left(w_{i}\right)=P_{W_{i}} T_{i}\left(w_{i}\right)=T_{i}\left(w_{i}\right)$. Logo, $m_{k}\left(w_{i}\right)=P_{W_{i}} m_{k} P_{W_{i}}\left(w_{i}\right)$, isto é, $m_{k}\left\lceil_{W_{i}}=P_{W_{i}} m_{k} P_{W_{i}}\right\rceil_{W_{i}}$, para todo $k \in \mathbb{N}$.

Como $\left(P_{W_{i}} m_{k} P_{W_{i}}\right)_{k}$ é uma sequência $w$-convergente a zero no espaço de dimensão finita $L\left(W_{i}\right)$ e $\left.P_{W_{i}} m_{k} P_{W_{i}}\right\rceil_{W_{i}} \stackrel{w}{\longrightarrow} 0$, então $\left.P_{W_{i}} m_{k} P_{W_{i}}\right\rceil_{W_{i}} \longrightarrow 0$. Para todo $i \leq p$, temos que

$$
P_{W_{i}} m_{k} P_{W_{i}}\left\lceil W_{i} \longrightarrow 0 \Longrightarrow P_{W_{i}} m_{k} P_{W_{i}}\left(w_{i}\right) \longrightarrow 0 \Longrightarrow m_{k}\left(w_{i}\right) \longrightarrow 0 \Longrightarrow \delta_{w_{i}}\left(m_{k}\right) \longrightarrow 0\right.
$$

Como $\delta_{v_{i}}\left(m_{k}\right) \longrightarrow 0$ e $\delta_{w_{i}}\left(m_{k}\right) \longrightarrow 0$, então existe $k_{0} \in \mathbb{N}$ tal que, para todo $i \leq p$ e todo 
$k \geq k_{0}$,

$$
\begin{aligned}
\left\|\delta_{v_{i}}\left(m_{k}\right)\right\|<\frac{\varepsilon}{3 p} & \Longrightarrow \sum_{i=1}^{p}\left\|\delta_{v_{i}}\left(m_{k}\right)\right\|<\frac{\varepsilon}{3} \quad \mathrm{e} \\
\left\|\delta_{v_{i}}\left(m_{k}\right)\right\|<\frac{\varepsilon}{3 p} & \Longrightarrow \sum_{i=1}^{p}\left\|\delta_{v_{i}}\left(m_{k}\right)\right\|<\frac{\varepsilon}{3}
\end{aligned}
$$

Assim, para todo $k \geq k_{0}$,

$$
\begin{aligned}
\left\|\delta_{h}\left(m_{k}\right)\right\| & \leq\left\|\delta_{h}\left(m_{k}\right)-\sum_{i=1}^{p}\left(\delta_{v_{i}}+\delta_{w_{i}}\right)\left(m_{k}\right)\right\|+\left\|\sum_{i=1}^{p}\left(\delta_{v_{i}}+\delta_{w_{i}}\right)\left(m_{k}\right)\right\| \\
& \leq\left\|\delta_{h}-\sum_{i=1}^{p}\left(\delta_{v_{i}}+\delta_{w_{i}}\right)\right\|\left\|m_{k}\right\|+\left\|\sum_{i=1}^{p} \delta_{v_{i}}\left(m_{k}\right)\right\|+\left\|\sum_{i=1}^{p} \delta_{w_{i}}\left(m_{k}\right)\right\| \\
& <\frac{\varepsilon}{3 \alpha} \alpha+\frac{\varepsilon}{3}+\frac{\varepsilon}{3}=\varepsilon .
\end{aligned}
$$

Logo, $\delta_{h}\left(m_{k}\right) \longrightarrow 0$.

Vejamos que $\gamma_{h}\left(m_{k}\right) \longrightarrow 0$. Para todo $k \in \mathbb{N}$,

$$
m_{k}^{t}=\left(\left(\oplus_{i=1}^{\infty} T_{i}^{(k)}\right)_{\infty} \oplus_{\infty}\left(\oplus_{i=1}^{\infty} S_{i}^{(k)}\right)_{\infty}\right)^{t}=\left(\oplus_{i=1}^{\infty}\left(T_{i}^{(k)}\right)^{t}\right)_{\infty} \oplus_{\infty}\left(\oplus_{i=1}^{\infty}\left(S_{i}^{(k)}\right)^{t}\right)_{\infty}
$$

Como $\left(T_{i}^{(k)}\right)^{t} \in L\left(V_{i}\right)$ e $\left(S_{i}^{(k)}\right)^{t} \in L\left(V_{i}\right)$, para todo $i \in \mathbb{N}$ e todo $k \in \mathbb{N}$, então $m_{k}^{t} \in M$, para todo $k \in \mathbb{N}$.

Analogamente, temos que $\gamma_{h}\left(m_{k}\right) \longrightarrow 0$.

Logo, os operadores avaliação $\delta_{h}$ e $\gamma_{h}$ são operadores DP1, para todo $h \in H$.

\subsection{Subálgebras Fechadas da Álgebra dos Operadores Compactos com Propriedade Dunford-Pettis Alternativa}

Para uma subálgebra de Banach fechada $A$ de $L(X)$, sendo $X$ um espaço de Banach e, para todo $S \in A$, denotaremos por $R_{S}$ e $L_{S}$ os operadores composição à direita e à esquerda respectivamente, dados por

$$
\begin{aligned}
R_{S}: & A \longrightarrow A \\
T & \longrightarrow R_{S}(T)=T S \\
L_{S}: A & \longrightarrow A \\
& T \longrightarrow L_{S}(T)=S T
\end{aligned}
$$

Vimos, na Seção 1.4, que os espaços $K(X)$ e $K(H)$ são álgebras de Banach (Exemplo 1.4.6). Ao utilizar os resultados da Seção 3.1, isto é, Proposição 3.1.1 e Teorema 3.1.14, veremos que uma subálgebra $A$ de $K(X)$, com mais algumas hipóteses sobre os espaços, possui a propriedade DP1 se, e somente se, os operadores composição à direita e à esquerda são operadores DP1.

Analogamente, ao utilizar o Teorema 3.2.7, veremos que uma subálgebra $A$ de $K(H)$, com mais algumas hipóteses sobre os espaços, possui a DP1 se, e somente se, os operadores composição à direita e à esquerda são operadores DP1.

Começaremos pelo estudo das subálgebras de $K(X)$. 
Proposição 3.3.1. Sejam $X$ um espaço de Banach reflexivo e A uma subálgebra fechada de $K(X)$. Se A possui a propriedade DP1, então os operadores $R_{S}, L_{S}$ são operadores DP1, para todo $S \in A$.

Demonstração. Seja $T_{n} \stackrel{w}{\longrightarrow} T$ em $A$, com $\left\|T_{n}\right\|=\|T\|=1$. Como $A$ tem a propriedade DP1, pela Proposição 3.1.1, segue que, para todo $x \in X$ e todo $g \in X^{*}$, os operadores avaliação

$$
\begin{array}{rlrl}
\delta_{x}: A & \longrightarrow X & \gamma_{g}: A & \longrightarrow X^{*} \\
U & \mapsto U(x) & U \mapsto U^{*}(g)
\end{array}
$$

são operadores DP1. Assim, para todo $x \in X$ e todo $g \in X^{*}$, temos que

$$
\begin{array}{r}
\left\|\delta_{x}\left(T_{n}\right)-\delta_{x}(T)\right\|=\left\|T_{n}(x)-T(x)\right\| \longrightarrow 0, \\
\left\|\gamma_{g}\left(T_{n}\right)-\gamma_{g}(T)\right\|=\left\|T_{n}^{*}(g)-T^{*}(g)\right\| \longrightarrow 0 .
\end{array}
$$

Para todo $S \in A$, temos que $S$ e $S^{*}$ são compactos. Pelo Lema 3.1.3, segue que $T_{n} S \longrightarrow T S$ e $T_{n}^{*} S^{*} \longrightarrow T^{*} S^{*}$

Então, $R_{S}\left(T_{n}\right) \longrightarrow R_{S}(T)$ e $\left\|T_{n}^{*} S^{*}-T^{*} S^{*}\right\|=\left\|S T_{n}-S T\right\| \longrightarrow 0$, isto é, $L_{S}\left(T_{n}\right) \longrightarrow L_{S}(T)$, para todo $S \in A$. Logo, $R_{S}$ e $L_{S}$ são operadores DP1.

A recíproca da Proposição 3.3.1 não vale em geral. Para obtermos uma recíproca será necessário acrescentar algumas hipóteses - trata-se do próximo teorema:

Teorema 3.3.2. Sejam X um espaço de Banach reflexivo com base de Schauder e A uma subálgebra fechada de $K(X)$ satisfazendo à propriedade $\mathcal{P}$. Suponhamos que os subespaços lineares gerados por $\{T(x): x \in X, T \in A\}$ e $\left\{T^{*}(f): f \in X^{*}, T \in A\right\}$ são densos em $X$ e $X^{*}$ respectivamente. Se os operadores $L_{T}, R_{T}: A \longrightarrow A$ são operadores $D P 1$, para todo $T \in A$, então A possui a propriedade DP1.

Demonstração. Pelo Corolário 3.1.15, temos que verificar que os operadores avaliação

$$
\begin{aligned}
\delta_{x}: A & \longrightarrow X & \gamma_{f}: A & \longrightarrow X^{*} \\
U & \longmapsto U(x) & U & \longmapsto U^{*}(f)
\end{aligned}
$$

são operadores DP1, para todo $x \in X$ e todo $f \in X^{*}$.

Fixemos $x \in X, f \in X^{*}$. Sejam $T_{n} \stackrel{w}{\longrightarrow} 0$ em $A$ e $S \in A$, com $\left\|T_{n}+S\right\|=\|S\|=1$. Mostraremos que $\delta_{x}\left(T_{n}\right) \longrightarrow 0$ e $\gamma_{f}\left(T_{n}\right) \longrightarrow 0$, isto é, $T_{n}(x) \longrightarrow 0$ e $T_{n}^{*}(f) \longrightarrow 0$.

Consideremos $\alpha=\sup _{n \in \mathbb{N}}\left\|T_{n}\right\|$.

Como $[T(x): x \in X, T \in A]$ é denso em $X$, existe $\left(x_{n}\right)_{n} \subset[T(x): x \in X, T \in A]$ tal que $x_{n} \longrightarrow$ $x$.

Dado $\varepsilon>0$, existe $n_{1} \in \mathbb{N}$ tal que

$$
\left\|x_{n}-x\right\|<\frac{\varepsilon}{2 \alpha}
$$

para todo $n \geq n_{1}$. Fixemos $k \geq n_{1}$. Como $x_{k} \in[T(x): x \in X, T \in A]$, existem $y_{1}, \ldots, y_{m} \in X$ e $S_{1}, \ldots, S_{m} \in A$ tais que 


$$
x_{k}=\sum_{i=1}^{m} S_{i}\left(y_{i}\right) \quad \text { e } \quad\left\|\sum_{i=1}^{m} S_{i}\left(y_{i}\right)-x\right\|<\frac{\varepsilon}{2 \alpha} .
$$

Então, para todo $n \in \mathbb{N}$,

$$
\left\|\sum_{i=1}^{m} T_{n} S_{i}\left(y_{i}\right)-T_{n}(x)\right\| \leq\left\|T_{n}\right\| \frac{\varepsilon}{2 \alpha} \leq \alpha \frac{\varepsilon}{2 \alpha}=\frac{\varepsilon}{2} .
$$

Como $R_{S_{i}}$ é um operador DP1, para todo $i=1, \ldots, m$, segue que $R_{S_{i}}\left(T_{n}\right) \longrightarrow 0$, isto é, $T_{n} S_{i} \longrightarrow 0$, para todo $i=1, \ldots, m$. Então, $\sum_{i=1}^{m} T_{n} S_{i}\left(y_{i}\right) \longrightarrow 0$. Assim, existe $n_{0} \in \mathbb{N}$ tal que, para todo $n \geq n_{0}$,

$$
\left\|\sum_{i=1}^{m} T_{n} S_{i}\left(y_{i}\right)\right\|<\frac{\varepsilon}{2}
$$

Por (3.5) e (3.6), segue que, para todo $n \geq n_{0}$

$$
\left\|T_{n}(x)\right\| \leq\left\|T_{n}(x)-\sum_{i=1}^{m} T_{n} S_{i}\left(y_{i}\right)\right\|+\left\|\sum_{i=1}^{m} T_{n} S_{i}\left(y_{i}\right)\right\|<\frac{\varepsilon}{2}+\frac{\varepsilon}{2}=\varepsilon .
$$

Logo, $T_{n}(x) \longrightarrow 0$.

Analogomente, considere $\beta=\sup _{n \in \mathbb{N}}\left\|T_{n}^{*}\right\|$. Como $\left[T^{*}(f): f \in X^{*}, T \in A\right]$ é denso em $X^{*}$, existe $\left(f_{n}\right)_{n} \subset\left[T^{*}(f): f \in X^{*}, T \in A\right]$ tal que $f_{n} \longrightarrow f$.

Dado $\varepsilon>0$, existe $n_{1} \in \mathbb{N}$ tal que

$$
\left\|f_{n}-f\right\|<\frac{\varepsilon}{2 \beta}
$$

para todo $n \geq n_{1}$. Fixemos $k \geq n_{1}$. Como $f_{k} \in\left[T^{*}(f): f \in X^{*}, T \in A\right]$, existem $g_{1}, \ldots, g_{m} \in X^{*} \mathrm{e}$ $U_{1}, \ldots, U_{m} \in A$ tais que

$$
f_{k}=\sum_{i=1}^{m} U_{i}^{*}\left(g_{i}\right) \quad \text { e } \quad\left\|\sum_{i=1}^{m} U_{i}^{*}\left(g_{i}\right)-f\right\|<\frac{\varepsilon}{2 \beta} .
$$

Então, para todo $n \in \mathbb{N}$,

$$
\left\|\sum_{i=1}^{m} T_{n}^{*} U_{i}^{*}\left(g_{i}\right)-U_{n}^{*}(f)\right\| \leq\left\|T_{n}^{*}\right\| \frac{\varepsilon}{2 \beta} \leq \beta \frac{\varepsilon}{2 \beta}=\frac{\varepsilon}{2} .
$$

Como $L_{U_{i}}$ é um operador DP1, para todo $i=1, \ldots, m$, segue que $L_{U_{i}}\left(T_{n}\right) \longrightarrow 0$, isto é, $U_{i} T_{n} \longrightarrow 0$, para todo $i=1, \ldots, m$. Então, $T_{n}^{*} U_{i}^{*} \longrightarrow 0$, para todo $i=1, \ldots, m$, que implica $\sum_{i=1}^{m} T_{n}^{*} U_{i}^{*}\left(g_{i}\right) \longrightarrow 0$. Assim, existe $n_{0} \in \mathbb{N}$ tal que, para todo $n \geq n_{0}$

$$
\left\|\sum_{i=1}^{m} T_{n}^{*} U_{i}^{*}\left(g_{i}\right)\right\|<\frac{\varepsilon}{2}
$$


Por (3.7) e (3.8), segue que, para todo $n \geq n_{0}$,

$$
\left\|T_{n}^{*}(f)\right\| \leq\left\|T_{n}^{*}(f)-\sum_{i=1}^{m} T_{n}^{*} U_{i}^{*}\left(g_{i}\right)\right\|+\left\|\sum_{i=1}^{m} T_{n}^{*} U_{i}^{*}\left(g_{i}\right)\right\|<\frac{\varepsilon}{2}+\frac{\varepsilon}{2}=\varepsilon .
$$

Logo, $T_{n}^{*}(f) \longrightarrow 0$. Assim, $\delta_{x}$ e $\gamma_{f}$ são DP1 e, portanto, $A$ tem a propriedade DP1.

Como consequência das Proposições 3.3.1 e 3.3.2, temos o corolário:

Corolário 3.3.3. Sejam X um espaço de Banach reflexivo com base de Schauder e A uma subálgebra fechada de $K(X)$ satisfazendo à propriedade $\mathcal{P}$. Suponhamos que os subespaços lineares gerados por $\{T(x): x \in X, T \in A\}$ e $\left\{T^{*}(f): f \in X^{*}, T \in A\right\}$ são densos em $X$ e $X^{*}$ respectivamente. Então, A tem a propriedade DP1 se, e somente se, os operadores $L_{T}, R_{T}: A \longrightarrow A$ são DP1.

Estudaremos um resultado análogo para subálgebras de $K(H)$.

Teorema 3.3.4. Sejam $H$ um espaço de Hilbert e $A$ uma subálgebra fechada de $K(H)$. Suponhamos que os subespaços lineares gerados por $\{T(h): h \in H, T \in A\}$ e $\left\{T^{t}(h): h \in H, T \in A\right\}$ são densos em H. Se os operadores $L_{T}, R_{T}: A \longrightarrow A$ são DP1, para todo $T \in A$, então A possui a propriedade $D P 1$.

Demonstração. Pelo Teorema 3.2.7, temos que verificar que os operadores avaliação

$$
\begin{aligned}
\delta_{h}: A & \longrightarrow H & \gamma_{h}: A & \longrightarrow H \\
U & \longmapsto U(h) & U & \longmapsto U^{t}(h)
\end{aligned}
$$

são operadores DP1, para todo $h \in H$.

Fixemos $h \in H$. Sejam $T_{n} \stackrel{w}{\longrightarrow} 0$ em $A, S \in A$ tais que $\left\|T_{n}+S\right\|=\|S\|=1$. Mostraremos que $\delta_{h}\left(T_{n}\right) \longrightarrow 0$ e $\gamma_{h}\left(T_{n}\right) \longrightarrow 0$, isto é, $T_{n}(h) \longrightarrow 0$ e $T_{n}^{t}(h) \longrightarrow 0$.

Consideremos $\alpha=\sup _{n \in \mathbb{N}}\left\|T_{n}\right\|$. Como $[T(h): h \in X, T \in A]$ é denso em $H$, existe $\left(h_{n}\right)_{n} \subset[T(h): h \in H, T \in A$ tal que $h_{n} \longrightarrow h$.

Dado $\varepsilon>0$, existe $n_{1} \in \mathbb{N}$ tal que

$$
\left\|h_{n}-h\right\|<\frac{\varepsilon}{2 \alpha}
$$

para todo $n \geq n_{1}$. Fixemos $k \geq n_{1}$. Como $h_{k} \in[T(h): h \in H, T \in A]$, existem $y_{1}, \ldots, y_{m} \in H$ e $S_{1}, \ldots, S_{m} \in A$ tais que

$$
h_{k}=\sum_{i=1}^{m} S_{i}\left(y_{i}\right) \quad \text { e } \quad\left\|\sum_{i=1}^{m} S_{i}\left(y_{i}\right)-h\right\|<\frac{\varepsilon}{2 \alpha} .
$$

Então, para todo $n \in \mathbb{N}$,

$$
\left\|\sum_{i=1}^{m} T_{n} S_{i}\left(y_{i}\right)-T_{n}(h)\right\| \leq\left\|T_{n}\right\| \frac{\varepsilon}{2 \alpha} \leq \alpha \frac{\varepsilon}{2 \alpha}=\frac{\varepsilon}{2} .
$$


Como $R_{S_{i}}$ é um operador DP1, para todo $i=1, \ldots, m$, segue que $R_{S_{i}}\left(T_{n}\right) \longrightarrow 0$, isto é, $T_{n} S_{i} \longrightarrow 0$, para todo $i=1, \ldots, m$. Então, $\sum_{i=1}^{m} T_{n} S_{i}\left(y_{i}\right) \longrightarrow 0$. Assim, existe $n_{0} \in \mathbb{N}$ tal que, para todo $n \geq n_{0}$

$$
\left\|\sum_{i=1}^{m} T_{n} S_{i}\left(y_{i}\right)\right\|<\frac{\varepsilon}{2}
$$

Por (3.9) e (3.10), para todo $n \geq n_{0}$, temos que

$$
\left\|T_{n}(h)\right\| \leq\left\|T_{n}(h)-\sum_{i=1}^{m} T_{n} S_{i}\left(y_{i}\right)\right\|+\left\|\sum_{i=1}^{m} T_{n} S_{i}\left(y_{i}\right)\right\|<\frac{\varepsilon}{2}+\frac{\varepsilon}{2}=\varepsilon .
$$

Logo, $T_{n}(h) \longrightarrow 0$.

De maneira análoga, vejamos que $T_{n}^{t}(h) \longrightarrow 0$. Consideremos $\beta=\sup _{n \in \mathbb{N}}\left\|T_{n}^{t}\right\|$. Como $\left[T^{t}(h): h \in H, T \in A\right]$ é denso em $H$, existe $\left(z_{n}\right)_{n} \subset\left[T^{t}(h): h \in H, T \in A\right]$ tal que $z_{n} \longrightarrow h$.

Dado $\varepsilon>0$, existe $n_{1} \in \mathbb{N}$ tal que

$$
\left\|z_{n}-h\right\|<\frac{\varepsilon}{2 \beta}
$$

para todo $n \geq n_{1}$. Fixemos $k \geq n_{1}$. Como $z_{k} \in\left[T^{t}(h): h \in H, T \in A\right]$, existem $w_{1}, \ldots, w_{m} \in H$ e $U_{1}, \ldots, U_{m} \in A$ tais que

$$
z_{k}=\sum_{i=1}^{m} U_{i}^{t}\left(w_{i}\right) \quad \text { e } \quad\left\|\sum_{i=1}^{m} U_{i}^{t}\left(w_{i}\right)-h\right\|<\frac{\varepsilon}{2 \beta} .
$$

Então, para todo $n \in \mathbb{N}$,

$$
\left\|\sum_{i=1}^{m} T_{n}^{t} U_{i}^{t}\left(w_{i}\right)-T_{n}^{t}(h)\right\| \leq\left\|T_{n}^{t}\right\| \frac{\varepsilon}{2 \beta} \leq \beta \frac{\varepsilon}{2 \beta}=\frac{\varepsilon}{2} .
$$

Como $L_{U_{i}}$ é um operador DP1, para todo $i=1, \ldots, m$, temos que $L_{U_{i}}\left(T_{n}\right) \longrightarrow 0$, isto é, $U_{i} T_{n} \longrightarrow 0$, para todo $i=1, \ldots, m$. Então, $T_{n}^{t} U_{i}^{t} \longrightarrow 0$, para todo $i=1, \ldots, m$, que implica $\sum_{i=1}^{m} T_{n}^{t} U_{i}^{t}\left(y_{i}\right) \longrightarrow 0$. Assim, existe $n_{0} \in \mathbb{N}$ tal que, para todo $n \geq n_{0}$,

$$
\left\|\sum_{i=1}^{m} T_{n}^{t} U_{i}^{t}\left(w_{i}\right)\right\|<\frac{\varepsilon}{2}
$$

Por (3.11) e (3.12), para todo $n \geq n_{0}$, temos que

$$
\left\|T_{n}^{t}(h)\right\| \leq\left\|T_{n}^{t}(h)-\sum_{i=1}^{m} T_{n}^{t} U_{i}^{t}\left(y_{i}\right)\right\|+\left\|\sum_{i=1}^{m} T_{n}^{t} U_{i}^{t}\left(w_{i}\right)\right\|<\frac{\varepsilon}{2}+\frac{\varepsilon}{2}=\varepsilon .
$$

Logo, $T_{n}^{t}(h) \longrightarrow 0$. Assim, $\delta_{h}$ e $\gamma_{h}$ são DP1 e, portanto, $A$ tem a propriedade DP1. 
Pelo Teorema 1.3.6, todo espaço de Hilbert $H$ é reflexivo. Assim, pela Proposição 3.3 .1 e pelo Teorema 3.3.4, temos o corolário:

Corolário 3.3.5. Sejam $H$ um espaço de Hilbert e $A$ uma subálgebra fechada de $K(H)$. Suponhamos que os subespaços lineares gerados por $\{T(h): h \in H, T \in A\}$ e $\left\{T^{t}(h): h \in H, T \in A\right\}$ são densos em H. Então, A tem a propriedade DP1 se, e somente se, os operadores $L_{T}, R_{T}: A \longrightarrow A$ são operadores DP1. 


\section{Capítulo 4}

\section{Propriedade Dunford-Pettis Alternativa em $C^{*}$-Âlgebras}

A equivalência das propriedades DP e DP1 em $C^{*}$-álgebras será estudada neste capítulo. Definições e resultados da teoria de $C^{*}$-álgebras serão apresentados nas primeiras seções. As principais referências utilizadas foram [28] e [29].

Neste capítulo, consideraremos sempre $\mathbb{K}=\mathbb{C}$.

\section{1 $C^{*}$-Álgebras}

O objetivo desta seção é apresentar conceitos básicos relativos à teoria de $C^{*}$-álgebras que serão necessários para o restante do capítulo. Alguns resultados serão demonstrados; outros serão acompanhados de referências para as demonstrações.

Começaremos pela definição de uma involução em uma álgebra, o que nos possibilitará definir *-álgebras, *-homomorfismos e alguns elementos específicos em uma *álgebra, como os elementos auto-adjuntos e projeções. Definiremos também *-álgebras de Banach e $C^{*}$-álgebras. Veremos os principais exemplos de $C^{*}$-álgebras, como os espaços $C(K), L(H)$ e $K(H)$.

O conceito de elemento positivo de uma $C^{*}$-álgebra é muito importante na teoria de operadores. Veremos a definição de elemento positivo, equivalências de tal definição, entre outras propriedades. Também definiremos quando dois elementos são ortogonais em uma $C^{*}$-álgebra.

Definiremos o quociente de uma $*$-álgebra por um ideal auto-adjunto e suas operações. Veremos que o quociente de uma $C^{*}$-álgebra por um ideal fechado é uma $C^{*}$-álgebra com a norma apropriada. Também definiremos uma $C^{*}$-subálgebra hereditária e veremos alguns exemplos.

Por fim, introduziremos, brevemente, a classe dos operadores traço, denotada por $\mathscr{L}_{1}(H)$, com o objetivo de usar que o dual do espaço $K(H)$ é isometricamente isomorfo a $\mathscr{L}_{1}(H)$. Esse resultado será utilizado fortemente na Seção 4.4, uma vez que trabalharemos com elementos do dual de $K(H)$ em algumas demonstrações.

Definição 4.1.1. Seja A uma álgebra. Uma involução em $A$ é uma aplicação $*: A \longrightarrow A$ satisfazendo às seguintes propriedades, para todo $a, b \in A$ e $\alpha \in \mathbb{C}$ :

(a) $\left(a^{*}\right)^{*}=a$;

(b) $(a b)^{*}=b^{*} a^{*}$; 
(c) $(\alpha a+b)^{*}=\bar{\alpha} a^{*}+b^{*}$.

Se $A$ é uma álgebra munida de uma involução, então dizemos que $A$ é uma $*$-álgebra. Se $A$ tem unidade, segue que $1_{A}^{*}=1_{A}$. De fato, temos que $1_{A}^{*}=1_{A} 1_{A}^{*}=\left(1_{A} 1_{A}^{*}\right)^{*}=\left(1_{A}^{*}\right)^{*}=1_{A}$. Denotaremos por $1_{A}$ ou apenas 1 a unidade de $A$.

Definição 4.1.2. Sejam A uma *álgebra e $a \in A$. Dizemos que:

(a) a é auto-adjunto, se $a^{*}=a$;

(b) a é projeção, se $a=a^{*}=a^{2}$.

Proposição 4.1.3. Seja $A$ uma *-álgebra. Então, para todo $a \in A$, existem únicos $b, c \in A$ autoadjuntos tais que $a=b+i c$.

Demonstração. Basta tomar $b=\frac{a+a^{*}}{2}$ e $c=\frac{a-a^{*}}{2 i}$.

Definição 4.1.4. Sejam $A$ e $B$ *álgebras e $\varphi: A \longrightarrow B$ uma aplicação linear. Dizemos que $\varphi$ é um *-homomorfismo se, para todo $a, b \in A$, temos que

$$
\varphi(a b)=\varphi(a) \varphi(b) \quad \text { e } \quad \varphi\left(a^{*}\right)=\varphi(a)^{*}
$$

Se $A$ e $B$ possuem unidade, exigimos que $\varphi\left(1_{A}\right)=1_{B}$. Um *-homomorfismo bijetivo é chamado *-isomorfismo.

Definição 4.1.5. Uma *-álgebra de Banach é uma álgebra de Banach A tal que $\left\|a^{*}\right\|=\|a\|$, para todo $a \in A$.

Definição 4.1.6. Uma $\mathbf{C}^{*}$-álgebra é uma *álgebra de Banach tal que $\left\|a^{*} a\right\|=\|a\|^{2}$, para todo $a \in A$. Se $A$ possui unidade $1_{A}$, dizemos que $A$ é uma $C^{*}$-álgebra com unidade.

Uma *-subálgebra fechada de uma $C^{*}$-álgebra A é denominada $\mathbf{C}^{*}$-subálgebra de A.

Proposição 4.1.7. Se A é uma $C^{*}$-álgebra com unidade e p é uma projeção não nula, então $\|p\|=1$. Em particular, 1 é projeção então $\|1\|=1$.

Demonstração. Temos que $\|p\|^{2}=\left\|p^{*} p\right\|=\left\|p^{2}\right\|=\|p\|$. Como $p \neq 0$, temos que $\|p\|=1$.

Vejamos alguns exemplos importantes de $C^{*}$-álgebras.

Exemplo 4.1.8. Seja $K$ um espaço topológico Hausdorff localmente compacto. Temos que $C_{0}(K)$ (definido no Exemplo 1.4.3), munido da involução dada pela conjugação, isto é, $f^{*}(x)=\overline{f(x)}$, é uma $C^{*}$-álgebra.

Se $K$ é compacto, então $C_{0}(K)$ é uma $C^{*}$-álgebra com unidade.

Exemplo 4.1.9. Seja $H$ um espaço de Hilbert. O espaço $L(H)$ com a involução dada pelo operador transposto (vide Definição 1.3.8 e Proposição 1.3.9), isto é, para todo $x, y \in H$,

$$
\langle T(x), y\rangle=\left\langle x, T^{t}(y)\right\rangle,
$$

é uma $C^{*}$-álgebra não comutativa com unidade, sendo esta o operador identidade $I d_{H}$. 
Exemplo 4.1.10. Seja $H$ um espaço de Hilbert. O espaço $K(H)$ é uma $C^{*}$-subálgebra de $L(H)$ e $I d_{H}$ é a unidade de $K(H)$ se, e somente se, $H$ tem dimensão finita. Além disso, $K(H)$ é auto-adjunto em $L(H)$, pois $K(H)$ coincide com o conjunto formado pelas involuções de todos os elementos de $K(H)$, isto é, $K(H)=\left\{T^{t}: T \in K(H)\right\}$, pois a involução é dada pelo operador transposto.

Na Seção 1.1, vimos que, para um espaço normado $X$, a aplicação canônica $C: X \longrightarrow X^{* *}$ (Definição 1.1.13) é linear, contínua, injetora e isometria. Assim, podemos identificar $X \operatorname{com} C(X)$ e ver $X$ como um subespaço de $X^{* *}$. Ao fazermos essa identificação, dizemos que $X$ está canonicamente imerso em $X^{* *}$. Vem, pois, o seguinte teorema:

Teorema 4.1.11. Seja $A^{* *}$ o bidual de uma $C^{*}$-álgebra $A$. Então $A^{* *}$ é uma $C^{*}$-álgebra. Além disso, $A$ é uma $C^{*}$-subálgebra de $A^{* *}$ quando $A$ é canonicamente imersa em $A^{* *}$.

(vide [30, p. 43])

A demonstração de que todo $*$-homomorfismo entre $C^{*}$-álgebras é contínuo utiliza o seguinte teorema, que envolve o raio espectral de um elemento auto-adjunto de uma $C^{*}$-álgebra. (Lembremos que as definições de espectro e raio espectral foram vistas na Seção 1.4.)

Teorema 4.1.12. Se a é um elemento auto-adjunto de uma $C^{*}$-álgebra $A$, então $r(a)=\|a\|$. (vide [28, p. 37])

Proposição 4.1.13. Sejam $A$ uma *álgebra de Banach, $B$ uma $C^{*}$-álgebra e $\varphi: A \longrightarrow B$ um *-homomorfismo. Então $\|\varphi(a)\| \leq\|a\|$, para todo $a \in A$, e, portanto, $\varphi$ é contínuo.

Demonstração. Seja $a \in A$. Então $\sigma(\varphi(a)) \subseteq \sigma(a)$. Assim, temos que

$$
\|\varphi(a)\|^{2}=\left\|\varphi(a)^{*} \varphi(a)\right\|=\left\|\varphi\left(a^{*} a\right)\right\|=r\left(\varphi\left(a^{*} a\right)\right) \leq r\left(a^{*} a\right)=\left\|a^{*} a\right\| \leq\|a\|^{2} .
$$

Logo, $\|\varphi(a)\| \leq\|a\|$

Proposição 4.1.14. Seja $A$ uma $C^{*}$-álgebra. Se a $\in A$ é auto-adjunto, então $\sigma(a) \subseteq \mathbb{R}$. (vide [28, p. 40])

A seguir estudaremos o conceito de elemento positivo de uma $C^{*}$-álgebra, que será muito utilizado nas próximas seções.

Definição 4.1.15. Seja $A$ uma $C^{*}$-álgebra. Um elemento $a \in A$ é denominado positivo se a é auto-adjunto e $\sigma(a) \subseteq \mathbb{R}_{+}$.

O próximo teorema nos traz duas caracterizações alternativas para elementos positivos.

Teorema 4.1.16. Seja $A$ uma $C^{*}$-álgebra com unidade. Dado a $\in A$, são equivalentes:

(a) a é positivo;

(b) existe um único elemento auto-adjunto e positivo $b \in A$ tal que $b^{2}=a$;

(c) existe $b \in A$ tal que $b^{*} b=a$.

(vide [28, p. 45]) 
Quando um elemento $a \in A$ é positivo, escrevemos $a \geq 0$. Denotamos por $a^{\frac{1}{2}}$ o único elemento positivo $b$ tal que $b^{2}=a$. Além disso, se $a, b \in A$ e $a-b \in A^{+}$, então escrevemos $a \geq b$.

Proposição 4.1.17. Seja A uma $C^{*}$-álgebra com unidade.

(a) Se $a \in A$ é auto-adjunto, então $a \leq\|a\|$ no sentido de que $\|a\| \cdot 1-a$ é positivo;

(b) Se $0 \leq a \leq b$, então $\|a\| \leq\|b\|$.

(vide [28, p. 46])

Introduziremos o conceito de elementos ortogonais em uma $C^{*}$-álgebra.

Definição 4.1.18. Seja A uma $C^{*}$-álgebra. Dizemos que $a, b \in A$ são ortogonais, denotando-se por $a \perp b$, se $a b=b a=a^{*} b=b a^{*}=0$.

Diretamente da definição, segue que

$$
\begin{aligned}
& 0=\left(a^{*} b\right)^{*}=b^{*} a, \\
& 0=\left(b a^{*}\right)^{*}=a b^{*}, \\
& 0=(a b)^{*}=b^{*} a^{*} \quad \mathrm{e} \\
& 0=(b a)^{*}=a^{*} b^{*} .
\end{aligned}
$$

Proposição 4.1.19. Sejam $A$ uma $C^{*}$-álgebra e $a, b \in A$. Se $a \perp b$, então $\|a+b\|=\max \{\|a\|,\|b\|\}$. Demonstração. Considere $A$ uma $C^{*}$-álgebra e $a, b \in A$ tais que $a \perp b$. Como $a \perp b$, temos as seguintes identidades:

$$
\begin{aligned}
& (a+b)^{*}(a+b)=\left(a^{*}+b^{*}\right)(a+b)=a^{*} a+a^{*} b+b^{*} a+b^{*} b=a^{*} a+b^{*} b \quad \text { e } \\
& \left((a+b)^{*}(a+b)\right)^{2}=\left(a^{*} a+b^{*} b\right)^{2}=\left(a^{*} a\right)^{2}+a^{*} a b^{*} b+b^{*} b a^{*} a+\left(b^{*} b\right)^{2}=\left(a^{*} a\right)^{2}+\left(b^{*} b\right)^{2} .
\end{aligned}
$$

Suponhamos que, para todo $n \in \mathbb{N}$, temos que

$$
\left((a+b)^{*}(a+b)\right)^{2 n}=\left(a^{*} a\right)^{2 n}+\left(b^{*} b\right)^{2 n} .
$$

Então

$$
\begin{aligned}
\left((a+b)^{*}(a+b)\right)^{2(n+1)} & =\left(a^{*} a+b^{*} b\right)^{2(n+1)}=\left(a^{*} a+b^{*} b\right)^{2 n}\left(a^{*} a+b^{*} b\right)^{2} \\
& =\left(\left(a^{*} a\right)^{2 n}+\left(b^{*} b\right)^{2 n}\right)\left(\left(a^{*} a\right)^{2}+\left(b^{*} b\right)^{2}\right) \\
& =\left(a^{*} a\right)^{2 n}\left(a^{*} a\right)^{2}+\left(a^{*} a\right)^{2 n}\left(b^{*} b\right)^{2}+\left(b^{*} b\right)^{2 n}\left(a^{*} a\right)^{2}+\left(b^{*} b\right)^{2 n}\left(b^{*} b\right)^{2} \\
& =\left(a^{*} a\right)^{2 n+2}+0+0+\left(b^{*} b\right)^{2 n+2} \\
& =\left(a^{*} a\right)^{2(n+1)}+\left(b^{*} b\right)^{2(n+1)} .
\end{aligned}
$$

Pela definição de $C^{*}$-álgebra (Definição 4.1.6), se $x \in A$ é auto-adjunto, então $\|x\|^{2}=\left\|x^{*} x\right\|=$ $\left\|x^{2}\right\|$. Segue, pelos Teoremas 1.4.8 e 4.1.12, que $\|x\|^{2 n}=\left\|x^{2 n}\right\|$. Assim, para todo $n \in \mathbb{N}$, temos que

$$
\begin{aligned}
\|a+b\|^{4 n} & =\left\|(a+b)^{*}(a+b)\right\|^{2 n}=\left\|\left((a+b)^{*}(a+b)\right)^{2 n}\right\|=\left\|\left(a^{*} a\right)^{2 n}+\left(b^{*} b\right)^{2 n}\right\| \\
& \leq\left\|\left(a^{*} a\right)^{2 n}\right\|+\left\|\left(b^{*} b\right)^{2 n}\right\|=\|a\|^{4 n}+\|b\|^{4 n} .
\end{aligned}
$$


Afirmamos que:

$$
\|a+b\|^{2} \leq \sqrt[2 n]{\|a\|^{4 n}+\|b\|^{4 n}} \stackrel{n \rightarrow \infty}{\longrightarrow} \max \{\|a\|,\|b\|\}^{2} .
$$

Para $0<\|a\|<\|b\|$, isto é, $0<\frac{\|a\|}{\|b\|}<1$, temos que

$$
\sqrt[2 n]{\|a\|^{4 n}+\|b\|^{4 n}}=\left(\|b\|^{4 n}\right)^{\frac{1}{2 n}}\left(\left(\frac{\|a\|}{\|b\|}\right)^{4 n}+1\right)^{\frac{1}{2 n}} \stackrel{n \rightarrow \infty}{\longrightarrow}\|b\|^{2},
$$

pois $\lim _{n \rightarrow \infty}\left(\frac{\|a\|}{\|b\|}\right)^{n}=0$.

Por outro lado, se $0<\|b\|<\|a\|$, então a convergência será para $\|a\|^{2}$. Portanto, $\sqrt[2 n]{\|a\|^{4 n}+\|b\|^{4 n}} \longrightarrow$ $\max \{\|a\|,\|b\|\}^{2}$, quando $n \rightarrow \infty$. Assim, $\|a+b\| \leq \max \{\|a\|,\|b\|\}$.

Para a outra desigualdade, temos que:

$$
\begin{gathered}
0 \leq a^{*} a \leq a^{*} a+b^{*} b=(a+b)^{*}(a+b) \quad \text { e } \\
0 \leq b^{*} b \leq a^{*} a+b^{*} b=(a+b)^{*}(a+b) .
\end{gathered}
$$

Pela Proposição 4.1.17 (b),

$$
\begin{array}{r}
\left\|a^{*} a\right\| \leq\left\|a^{*} a+b^{*} b\right\| \quad \mathrm{e} \\
\left\|b^{*} b\right\| \leq\left\|a^{*} a+b^{*} b\right\| .
\end{array}
$$

Então, $\|a+b\|^{2}=\left\|(a+b)^{*}(a+b)\right\|=\left\|a^{*} a+b^{*} b\right\| \geq \max \left\{\left\|a^{*} a\right\|,\left\|b^{*} b\right\|\right\}=\max \{\|a\|,\|b\|\}$.

Estudaremos a definição de quociente de uma $C^{*}$-álgebra por um ideal e alguns resultados de quocientes importantes para o nosso trabalho.

Se $I$ é um ideal auto-adjunto, isto é, $I=\left\{x^{*}: x \in I\right\}$, de uma *-álgebra $A$, então o quociente $A / I$ é uma *-álgebra com a multiplicação e involução dadas, respectivamente, por $(a+I)(b+I)=a b+I$ e $(a+I)^{*}=a^{*}+I$. A aplicação quociente $\pi$ de $A$ sobre $A / I$ é uma aplicação tal que $\pi(a)=a+I$.

Teorema 4.1.20. Seja I um ideal fechado em uma $C^{*}$-álgebra $A$, então:

(a) I é auto-adjunto e, portanto, uma $C^{*}$-subálgebra de $A$;

(b) o quociente $A / I$ é uma $C^{*}$-álgebra com as operações usuais e a norma

$$
\|a+I\|=\inf _{b \in I}\|a+b\| .
$$

(vide [28, p. 79])

Se $I$ é um ideal fechado em uma $C^{*}$-álgebra $A$, a aplicação quociente $\pi: A \longrightarrow A / I$ é um *-homomorfismo sobrejetor e Nuc $\pi=I$.

Proposição 4.1.21. Sejam $A$ uma $C^{*}$-álgebra separável, $I$ um ideal fechado de $A$ e $B$ uma $C^{*}$ álgebra isomorfa a $A / I$. Considere $\pi: A \longrightarrow B$ a aplicação quociente, isto é, a composição do 
isomorfismo de $A / I$ em $B$ com a aplicação quociente de $A$ em $A / I$. Se $b_{n} \stackrel{w}{\longrightarrow} 0$ em $B$, então existe $\left(a_{n}\right)_{n}$ uma sequência em $A$ tal que $\pi\left(a_{n}\right)=b_{n}$ e $a_{n} \stackrel{w}{\longrightarrow} 0$.

(vide [11])

Proposição 4.1.22. Sejam $A$ uma $C^{*}$-álgebra e $I$ um ideal fechado de $A$. Se $x, y \in A^{+}$, com $x y \in I$, então existem $a, b \in I$ tais que $(x-a)(y-b)=0$ e $(x-a),(y-b) \in A^{+}$.

(vide Proposição 2.3 e Corolário 2.4 em [3])

Teorema 4.1.23. Se $A$ e $B$ são $C^{*}$-álgebras e $\varphi: A \longrightarrow B$ é um *-homomorfismo injetivo, então $\varphi$ é uma isometria.

(vide [28, p. 80])

Proposição 4.1.24. Sejam $A$ e $B C^{*}$-álgebras e $\varphi: A \longrightarrow B$ um *-homomorfismo. Então Nuc $\varphi$ é ideal auto-adjunto e $\varphi(A)=\operatorname{Im} \varphi$ é uma $C^{*}$-subálgebra de $B$.

(vide [28, p. 81])

Introduziremos o conceito de $C^{*}$-álgebra hereditária, pois ele será importante na demonstração de que as propriedades DP e DP1 são equivalentes em $C^{*}$-álgebras.

Definição 4.1.25. Uma $C^{*}$-subálgebra $B$ de uma $C^{*}$-álgebra $A$ é dita hereditária se, para todos $a \in A$ e $b \in B$ satisfazendo $a \leq a \leq b$, temos que $a \in B$.

Claramente, 0 e $A$ são $C^{*}$-subálgebras hereditárias de $A$ e qualquer intersecção de $C^{*}$-subálgebras hereditárias é também hereditária.

O exemplo a seguir trata de um tipo especial de $C^{*}$-subálgebra hereditária.

Exemplo 4.1.26. Se $p$ é uma projeção em uma $C^{*}$-álgebra $A$, isto é, $p=p^{*}=p^{2}$, então a $C^{*}$ subálgebra $p A p=\{$ pap : $a \in A\}$ é hereditária. De fato, seja $b \in A$ tal que $0 \leq b \leq p a p$, para algum $a \in A$. Segue da Proposição 4.1.16 que $p$ é positivo, então, pelas Proposições 4.1.17 (a) e 4.1.7, temos que $0 \leq p \leq\|p\|=1$. Logo, $0 \leq 1-p$. Assim,

$$
0 \leq(1-p) b(1-p) \leq(1-p) \operatorname{pap}(1-p)
$$

Temos ainda que

$$
(1-p) \operatorname{pap}(1-p)=\left(p a p-p^{2} a p\right)(1-p)=0(1-p)=0
$$

Então, $(1-p) b(1-p)=0$. Lembremos que $b$ e $b^{\frac{1}{2}}$ são positivos e, portanto, auto-adjuntos, então

$$
\left\|b^{\frac{1}{2}}(1-p)\right\|^{2}=\left\|\left(b^{\frac{1}{2}}(1-p)\right)^{*} b^{\frac{1}{2}}(1-p)\right\|=\left\|(1-p)^{*}\left(b^{\frac{1}{2}}\right)^{*} b^{\frac{1}{2}}(1-p)\right\|=\|(1-p) b(1-p)\|=0 .
$$

Segue que

$$
b^{\frac{1}{2}}(1-p)=0 \Rightarrow b(1-p)=0 \Rightarrow b=b p \Rightarrow p b=p b p .
$$

Mas, $b=b^{*}=(b p)^{*}=p^{*} b^{*}=p b$. Então, $b=p b=p b p \in p A p$. Logo, $p A p$ é hereditária.

Outros dois exemplos de $C^{*}$-álgebras hereditárias serão vistos na Seção 4.4 - no Lema 4.4 .14 e na Proposição 4.4.16. 
O próximo teorema apresenta outra caracterização de $C^{*}$-álgebra hereditária que será usada na Seção 4.4. Este teorema também generaliza o Exemplo 4.1.26.

Teorema 4.1.27. Sejam $A$ uma $C^{*}$-álgebra e $B$ uma $C^{*}$-subálgebra de $A$. Então, $B$ é hereditária em $A$ se, e somente se, bac $\in B$, para todo $b, c \in B$ e $a \in A$.

(vide [28, p. 84])

Corolário 4.1.28. Se A é uma $C^{*}$-álgebra e a $\in A^{+}$, então $\overline{a A a}$ é uma $C^{*}$-subálgebra hereditária de $A$ gerada por $a$, sendo $\overline{a A a}$ o fecho do conjunto $a A a=\{a b a: b \in A\}$. Em particular, $a \in \overline{a A a}$.

(vide [28, p. 85])

Introduziremos a classe dos operadores traço, que é um tópico muito estudado na teoria de operadores. Esses operadores serão relevantes, pois, na Seção 4.4, precisaremos trabalhar com o dual do $K(H)$ e utilizaremos o fato de que o dual de $K(H)$ é isometricamente isomorfo à classe dos operadores traço.

Definição 4.1.29. Seja $H$ um espaço de Hilbert.

(a) Um operador $A: H \longrightarrow H$ é um operador traço se existe uma base ortonormal $\mathscr{B}$ de $H$ tal que $\sum_{x \in \mathscr{B}}\langle|A| x, x\rangle<\infty$, em que $|A|=\left(A^{*} A\right)^{\frac{1}{2}}$. O conjunto dos operadores traço de $H$ será denotada por $\mathscr{L}_{1}(H)$. Seja $A \in \mathscr{L}_{1}(H)$, definimos a norma traço de A por

$$
\|A\|_{1}=\sum_{x \in \mathscr{B}}\langle|A| x, x\rangle
$$

(b) Se $A \in \mathscr{L}_{1}(H)$ e $\mathscr{B}$ é uma base ortonormal de $H$, definimos o traço de $A$ por

$$
\operatorname{tr} A=\sum_{x \in \mathscr{B}}\langle A x, x\rangle
$$

Seja $A \in \mathscr{L}_{1}(H)$. Definimos $\Phi_{A}: K(H) \longrightarrow \mathbb{C}$ por

$$
\Phi_{A}(C)=\operatorname{tr}(C A)=\operatorname{tr}(A C),
$$

para todo $C \in K(H)$. Temos que $\Phi_{A}$ é um funcional linear em $K(H)$.

Teorema 4.1.30. A aplicação $A \longmapsto \Phi_{A}$ é um isomorfismo isométrico de $\mathscr{L}_{1}(H)$ sobre $K(H)^{*}$, isto é, o dual de $K(H)$.

(vide [28, p. 124])

\subsection{Teoria das Representações e o Teorema de Gelfand-Naimark- Segal}

Um resultado muito importante em $C^{*}$-álgebras, conhecido como Teorema de Gelfand-NaimarkSegal, diz que toda $C^{*}$-álgebra pode ser identificada com uma $C^{*}$-subálgebra de $L(H)$, para $H$ um 
espaço de Hilbert. Tal identificação é feita utilizando o conceito de representação, que será estudado nesta seção.

Os seguintes tópicos serão abordados: (i) representação de uma *álgebra; (ii) representação universal e o Teorema de Gelfand-Naimark-Segal; (iii) representação irredutível; (iv) álgebra de Fermion; e (v) Teorema de Gelfand-Naimark para $C^{*}$-álgebras comutativas.

Definição 4.2.1. Uma representação de uma *-álgebra $A$ é um par $(\pi, H)$, em que $H$ é um espaço de Hilbert e $\pi: A \longrightarrow L(H)$ é um *-homomorfismo. Se A tem unidade, então $\pi\left(1_{A}\right)=I d_{L(H)}$.

Dizemos que $\pi$ é fiel se $\pi$ for injetor, isto é, $\pi(a)=0$ se, e somente se, $a=0$. E dizemos que a representação tem dimensão finita se $H$ tem dimensão finita. Analogamente, dizemos que a representação tem dimensão infinita se $H$ tem dimensão infinita.

Observemos que, como $L(H)$ é uma $C^{*}$-álgebra, uma representação $\pi$ de uma $C^{*}$-álgebra $A$ é um $*$-homomorfismo entre $C^{*}$-álgebras. Portanto, pela Proposição 4.1.13, temos que $\|\pi(a)\| \leq\|a\|$, para todo $a \in A$, isto é, $\pi$ é contínua. Se $\pi$ for fiel, então, pelo Teorema 4.1.23, $\pi$ é uma isometria de $A$ em uma $C^{*}$-subálgebra de $L(H)$.

A soma direta de representações de uma $C^{*}$-álgebra $A$ também é uma representação de $A$. De fato, dada uma família de representações $\left(\pi_{\lambda}, H_{\lambda}\right)_{\lambda \in \Lambda}$ podemos obter uma representação $(\pi, H)$ de $A$, sendo $H=\bigoplus_{\lambda \in \Lambda} H_{\lambda}$ e $\pi(a)\left(\left(x_{\lambda}\right)_{\lambda \in \Lambda}\right)=\left(\pi_{\lambda}(a)\left(x_{\lambda}\right)\right)_{\lambda \in \Lambda}$, para todo $a \in A$ e $\left(x_{\lambda}\right)_{\lambda \in \Lambda} \in \bigoplus_{\lambda \in \Lambda} H_{\lambda}$.

Definição 4.2.2. Seja $A$ uma $C^{*}$-álgebra. Um funcional $\tau$ de $A$ é chamado de estado se $\tau\left(A^{+}\right) \subset$ $\mathbb{R}_{+} e\|\tau\|=1$.

A representação universal de $A$, denotada por $\left(\pi_{u}, H_{u}\right)$, é a soma direta de todas as representações $\left(\pi_{\tau}, H_{\tau}\right)$, para todo $\tau$ estado de $A$.

Teorema 4.2.3 (Gelfand-Naimark-Segal). Se A é uma $C^{*}$-álgebra, então a representação uni$\operatorname{versal}\left(\pi_{u}, H_{u}\right)$ de $A$ é fiel.

(vide [28, p. 94])

Pelo Teorema 4.1.23, sabemos que se $\pi_{u}$ é fiel, então $\pi_{u}$ é uma isometria. Segue do Teorema de Gelfand-Naimark-Segal que toda $C^{*}$-álgebra é isometricamente isomorfa a uma $C^{*}$-subálgebra de $L(H)$, para $H$ um espaço de Hilbert. Assim, podemos sempre enxergar uma $C^{*}$-álgebra como uma $C^{*}$-subálgebra de $L(H)$.

Quando a $C^{*}$-álgebra é comutativa, temos outra caracterização que é dada pelo Teorema de Gelfand-Naimark. O Teorema de Gelfand-Naimark nos mostra que toda $C^{*}$-álgebra comutativa é isometricamente isomorfa a $C_{0}(K)$, para $K$ um espaço localmente compacto. Em outras palavras, toda $C^{*}$-álgebra comutativa é essencialmente a álgebra das funções contínuas que tendem a zero no infinito sobre um espaço topológico localmente compacto Hausdorff. Além disso, se a $C^{*}$-álgebra possui unidade, então ela é a álgebra das funções contínuas sobre um compacto.

A seguir, estudaremos o conceito de representação irredutível que será importante para provar a equivalência das propriedades DP e DP1.

Definição 4.2.4. Seja $H$ um espaço de Hilbert. Um subespaço fechado $M$ de $H$ é invariante para um subconjunto $N$ de $L(H)$ se $T(M) \subset M$, para todo operador $T \in N$.

Uma representação $(\pi, H)$ de uma *-álgebra A é dita topologicamente irredutivel se os únicos subespaços fechados invariantes por $\pi(A)$ de $H$ são os triviais, isto é, 0 e o próprio $H$. 
Enunciaremos alguns resultados de representações irredutíveis, que serão importantes na Seção 4.4, e deixaremos referências para as demonstrações.

Teorema 4.2.5. Seja $B$ uma $C^{*}$-subálgebra hereditária de uma $C^{*}$-álgebra $A$. Para cada representação irredutível $(\pi, H)$ de $A$ tal que $B \not \subset N u c \pi$, temos que $\left(\pi \uparrow_{B}, \pi(B) H\right)$ é uma representação irredutivel de $B$.

(vide [29, p. 93])

Lema 4.2.6. Seja $A$ uma $C^{*}$-álgebra. Se $(\pi, H)$ é uma representação irredutivel de $A$ tal que $\pi(A) \cap K(H) \neq 0$, então $K(H) \subset \pi(A)$.

(vide [29, 192])

Na Seção 4.4, será visto que uma $C^{*}$-álgebra $A$ tem a propriedade DP se, e somente se, toda representação irredutível de $A$ tem dimensão finita. A demonstração desse resultado utiliza o conceito de $C^{*}$-álgebra de tipo I, que definimos:

Definição 4.2.7. Dizemos que uma $C^{*}$-álgebra $A$ é do tipo I se, para todo ideal fechado $J$ de $A$, existe um elemento não nulo $x \in A / J$ tal que a $C^{*}$-subálgebra $\overline{x(A / J) x}$ é comutativa.

Teorema 4.2.8. Se A é uma $C^{*}$-álgebra do tipo I, então, para toda representação irredutível $(\pi, H)$ de $A$, temos que $K(H) \subset \pi(A)$.

(vide [29, 192])

A álgebra de Fermion terá um papel importante para demonstrar a equivalência das propriedades DP e DP1. Dois resultados importantes a seguir são que a álgebra de Fermion contém uma cópia complementada de $K\left(\ell_{2}\right)$ e que toda $C^{*}$-álgebra que não é do tipo I está relacionada com a álgebra de Fermion por um *-homomorfismo. A álgebra de Fermion é um tipo particular de álgebra de Glimm. Definiremos álgebras de Glimm, mas não trabalharemos com sua estrutura - estamos apenas interessadas nos resultados mencionados. Para um estudo mais detalhado, vide [29, p. 205] e [6, p. $156]$.

Definição 4.2.9. Um sistema indutivo de $C^{*}$-álgebras é um conjunto $\left\{\left(A_{i}, \phi_{i j}\right): i, j \in \Omega, i \leq j\right\}$, sendo $\Omega$ um conjunto dirigido, $A_{i} C^{*}$-álgebras e $\phi_{i j} *$-homomorfismos de $A_{i}$ em $A_{j}$, tal que $\phi_{i k}=$ $\phi_{j k} \circ \phi_{i j}$, para $i \leq j \leq k$.

Definição 4.2.10. Seja $M_{m}$ a $C^{*}$-álgebra das matrizes $m \times m$ identificadas com $L\left(H_{m}\right)$. Suponha que $l: M_{m} \longrightarrow M_{n}$ é um *-homomorfismo de $M_{m}$ em $M_{n}$ tal que $l\left(1_{M_{m}}\right)=1_{M_{n}}$. Temos que $l$ é injetor.

Dada uma sequência $\{m(n): n \in \mathbb{N}\}$ de números naturais maiores ou iguais a 1 , seja $m(n)$ ! = $\prod_{k=1}^{n} m(k)$. Considere o sistema indutivo

$$
M_{m(1) !} \stackrel{l}{\rightarrow} M_{m(2) !} \stackrel{l}{\rightarrow} \cdots \stackrel{l}{\rightarrow} M_{m(n) !} \stackrel{l}{\rightarrow} \cdots
$$

O limite indutivo $M_{\infty}=\bigcup M_{m(n)}$ ! satisfaz a todos os axiomas para uma $C^{*}$-álgebra, exceto a completude. Denotaremos por $A_{\infty}$ o completamento de $M_{\infty}$. Então, $A_{\infty}$ é uma $C^{*}$-álgebra que chamamos de álgebra de Glimm de posto $\{m(n)\}$. A álgebra de Fermion é a álgebra de Glimm com $m(n)=2$, para todo $n$, denotada por $\otimes M_{2}$. 
Teorema 4.2.11. A álgebra de Fermion $\otimes M_{2}$ contém uma cópia complementada de $K\left(\ell_{2}\right)$. (vide [4])

Teorema 4.2.12. Para cada $C^{*}$-álgebra $A$ que não é do tipo I e toda álgebra de Glimm $A_{\infty}$, existem uma $C^{*}$-subálgebra $B$ de $A$ e um *-homomorfismo sobrejetor de $B$ sobre $A_{\infty}$.

(vide [29, p. 221])

Em particular, a álgebra de Fermion é uma álgebra de Glimm. Então, pelo teorema anterior, para cada $C^{*}$-álgebra $A$ que não é do tipo I, existem uma $C^{*}$-subálgebra $B$ de $A$ e um $*$-homomorfismo sobrejetor de $B$ sobre $\otimes M_{2}$. Temos, assim, o seguinte corolário:

Corolário 4.2.13. Para cada $C^{*}$-álgebra $A$ que não é do tipo $I$, existem uma $C^{*}$-subálgebra $B$ de $A$ e um ideal fechado $J$ em $B$ tais que $B / J$ é isomorfo à álgebra de Fermion $\otimes M_{2}$.

\section{3 Álgebras de von Neumann}

Um dos objetivos da Seção 4.4 é provar a equivalência das propriedades DP e DP1 em $C^{*}$ álgebras. Para tal serão necessários alguns resultados de álgebras de von Neumann. Apesar de o título desta seção ser álgebras de von Neumann, esta dissertação não tem como objetivo estudar profundamente tais álgebras. Assim, nos restringiremos apenas a algumas definições e resultados que serão utilizados na última seção. Os resultados serão enunciados e deixaremos referências para as demonstrações.

Definição 4.3.1. Sejam A uma álgebra e B um subconjunto de A. Definimos o comutante de $B$ como

$$
B^{\prime}=\{x \in A: x y=y x, \text { para todo } y \in B\}
$$

Observamos que $B^{\prime}$ é uma subálgebra de $A$. O bicomutante $B^{\prime \prime}$ de $B$ é $\left(B^{\prime}\right)^{\prime}$ e, analogamente, $B^{\prime \prime \prime}=\left(B^{\prime \prime}\right)^{\prime}$. Sempre temos que $B \subseteq B^{\prime \prime}$ e $B^{\prime}=B^{\prime \prime \prime}$. Se $A$ é uma álgebra normada, então $B^{\prime}$ é fechado. Se $A$ é uma *-álgebra e $B$ é auto-adjunto, então $B^{\prime}$ é uma $*$-subálgebra de $A$.

Definição 4.3.2. Seja $H$ um espaço de Hilbert. Dizemos que $\mathcal{M}$ é uma álgebra de von Neumann em $H$ se $\mathcal{M}$ é uma $*$-subálgebra de $L(H)$ tal que $\mathcal{M}=\mathcal{M}^{\prime \prime}$.

Seguem alguns exemplos de álgebras de von Neumann.

Exemplo 4.3.3. $L(H)$ é uma álgebra de von Neumann. Se $\left(H_{\lambda}\right)_{\lambda \in \Lambda}$ é uma família de espaços de Hilbert e $\mathcal{M}_{\lambda}$ é uma álgebra de von Neumann em $H_{\lambda}$, para cada índice $\lambda$, então a soma direta $\oplus_{\lambda} \mathcal{M}_{\lambda}$ é uma álgebra de von Neumann em $\oplus_{\lambda} H_{\lambda}$.

Exemplo 4.3.4. Se $A$ é uma *-álgebra em $H$, então o comutante $A^{\prime}$ e o bicomutante $A^{\prime \prime}$ são álgebras de von Neumann.

Exemplo 4.3.5. Se $H$ é um espaço de Hilbert de dimensão infinita, então $K(H)$ não é uma álgebra de von Neumann em $H$.

Toda álgebra de von Neumann possui unidade. Esse fato será usado na demonstração da equivalência entre as propriedades DP e DP1 em $C^{*}$-álgebras. 
Teorema 4.3.6. Se $\mathcal{M}$ é uma álgebra de von Neumann não nula, então $\mathcal{M}$ tem unidade. (vide [28, p. 118])

Na última seção, utilizaremos o conceito de projeção imagem definida abaixo.

Definição 4.3.7. Seja $u \in L(H)$. Definimos a projeção imagem de u, denotada por $\mathcal{R}(u)$, como a projeção de $H$ em $\overline{u(H)}$.

Observação 4.3.8. A projeção imagem de um elemento auto-adjunto $u$ é a menor projeção tal que $\mathcal{R}(u) u=u \mathcal{R}(u)=u$, isto é, se $p$ e $q$ são projeções dizemos que $p$ é menor do que $q$ se $q-p$ é positivo.

Teorema 4.3.9. Se $\mathcal{M}$ é uma álgebra de von Neumann, então $\mathcal{M}$ contém as projeções imagem de todos os seus elementos.

(vide [28, p. 119])

Proposição 4.3.10. Sejam $A$ uma $C^{*}$-álgebra, $a \in A$ um elemento positivo e $A(x):=\overline{x A x}$, isto é, a $C^{*}$-subálgebra de $A$ gerada por $x$. Então, $A(x)^{* *}=\mathcal{R}(x) A^{* *} \mathcal{R}(x)$, em que $\mathcal{R}(x)$ é a projeção imagem de $x$ em $A^{* *}$.

(vide [10])

Um exemplo de álgebra de von Neumann muito importante é o bidual de uma $C^{*}$-álgebra. Foi visto na Seção 4.1 que o bidual de toda $C^{*}$-álgebra é uma $C^{*}$-álgebra. A próxima proposição diz que o bidual de toda $C^{*}$-álgebra é uma álgebra de von Neumann.

Proposição 4.3.11. Seja $A$ uma $C^{*}$-álgebra. O bicomutante de $A$ é isomorfo, como espaço de Banach, ao bidual de $A$, ou seja, $A^{\prime \prime}$ é isomorfo a $A^{* *}$.

(vide [29, p. 60])

Exemplo 4.3.12. O bidual de uma $C^{*}$-álgebra é uma álgebra de von Neumann. De fato, seja $A$ uma $C^{*}$-álgebra, pelo Teorema 4.1.11, temos que $A^{* *}$ é uma $C^{*}$-álgebra. Pelo Exemplo 4.3.4, $A^{\prime \prime}$ é uma álgebra de von Neumann e, pela Proposição 4.3.11, $A^{\prime \prime}$ e $A^{* *}$ são isomorfos. Logo, $A^{* *}$ é uma álgebra de von Neumann.

Os próximos dois teoremas serão usados no início da seção seguinte, para mostrarmos um resultado equivalente à definição da propriedade DP em $C^{*}$-álgebras.

Teorema 4.3.13. Seja $\mathcal{M}$ uma álgebra de von Neumann. Então o pré-dual $\mathcal{M}_{*}$ de $\mathcal{M}$ é $w$ sequencialmente completo.

(vide [32, p. 148])

Teorema 4.3.14. Sejam $X$ um espaço de Banach w-sequencialmente completo e $A$ uma $C^{*}$-álgebra. Se $T \in L(A, X)$, então $T$ é w-compacta.

(vide [2])

\subsection{Equivalência das Propriedades Dunford-Pettis e Dunford-Pettis Alternativa em $C^{*}$-Álgebras}

O objetivo desta seção é estudar as propriedades DP e DP1 em $C^{*}$-álgebras e observar que elas são equivalentes em $C^{*}$-álgebras. 
Alguns resultados equivalentes às definições das propriedades DP e DP1 em $C^{*}$-álgebras serão estudados e, como consequência dessas equivalências, veremos que as propriedades DP e DP1 são preservadas em $C^{*}$-subálgebras de uma $C^{*}$-álgebra. Será visto que $K(H)$ não possui a propriedade DP1 e, portanto, não possui a propriedade DP. Em decorrência disso, seguirá que $L(H)$ não possui as propriedades DP e DP1.

Entre esses resultados temos: (i) uma $C^{*}$-álgebra $A$ possui a propriedade DP se, e somente se, toda representação irredutível de $A$ tem dimensão finita; (ii) se uma $C^{*}$-álgebra $A$ possui a propriedade DP1, então toda representação irredutível de $A$ tem dimensão finita. Como consequência desses dois resultados, seguirá que uma $C^{*}$-álgebra possui a propriedade DP se, e somente se, possui a propriedade DP1.

Por fim, será vista uma caracterização para as propriedades DP e DP1 em $C^{*}$-subálgebras da álgebra $K(H)$.

O seguinte lema será necessário para o estudo de um resultado equivalente à definição da propriedade DP em $C^{*}$-álgebras.

Lema 4.4.1. Sejam A uma $C^{*}$-álgebra e $\left(x_{n}\right)_{n}$ uma sequência em $A$. Então, $x_{n} \stackrel{w}{\longrightarrow} x$ em $A$ se, e somente se, $x_{n}^{*} \stackrel{w}{\longrightarrow} x^{*}$ em $A$.

Demonstração. Para cada $f \in A^{*}$, definimos $g(x)=\overline{f\left(x^{*}\right)}$, sendo $\overline{f\left(x^{*}\right)}$ o conjugado do número complexo $f\left(x^{*}\right)$. Assim, temos que $g \in A^{*}$.

$(\Rightarrow)$ Sejam $x_{n} \stackrel{w}{\longrightarrow} x$ em $A$ e $f \in A^{*}$. Como $g \in A^{*}$, temos que $g\left(x_{n}\right) \longrightarrow g(x)$. Então,

$$
g\left(x_{n}\right) \longrightarrow g(x) \Rightarrow \overline{f\left(x_{n}^{*}\right)} \longrightarrow \overline{f\left(x^{*}\right)} \Rightarrow f\left(x_{n}^{*}\right) \longrightarrow f\left(x^{*}\right)
$$

Logo, $x_{n}^{*} \stackrel{w}{\longrightarrow} x^{*}$.

$(\Leftarrow)$ Analogamente, sejam $x_{n}^{*} \stackrel{w}{\longrightarrow} x^{*}$ em $A$ e $f \in A^{*}$. Como $g \in A^{*}$, temos que $g\left(x_{n}^{*}\right) \longrightarrow g\left(x^{*}\right)$. Então,

$$
g\left(x_{n}^{*}\right) \longrightarrow g\left(x^{*}\right) \Rightarrow \overline{f\left(\left(x_{n}^{*}\right)^{*}\right)} \longrightarrow \overline{f\left(\left(x^{*}\right)^{*}\right)} \Rightarrow f\left(x_{n}\right) \longrightarrow f(x)
$$

Logo, $x_{n} \stackrel{w}{\longrightarrow} x$.

Teorema 4.4.2. Seja A uma $C^{*}$-álgebra. As seguintes condições são equivalentes:

(a) A tem a propriedade DP;

(b) Se $x_{n} \stackrel{w}{\longrightarrow} 0$ em A, então $x_{n}^{*} x_{n} \stackrel{w}{\longrightarrow} 0$;

(c) Se $x_{n} \stackrel{w}{\longrightarrow} 0$ em A, então $x_{n} x_{n}^{*} \stackrel{w}{\longrightarrow} 0$;

(d) Se $x_{n} \stackrel{w}{\longrightarrow} 0$ em A, então $x_{n}^{*} x_{n}+x_{n} x_{n}^{*} \stackrel{w}{\longrightarrow} 0$.

Demonstração. (a) $\Rightarrow$ (b) Sejam $x_{n} \stackrel{w}{\longrightarrow} 0$ em $A$ e $f \in A^{*}$. Mostraremos que $f\left(x_{n}^{*} x_{n}\right) \longrightarrow 0$. 
Definimos

$$
\begin{aligned}
T: A & \longrightarrow A^{*} \\
a \longrightarrow T a: A & \longrightarrow \mathbb{K} \\
x & \longrightarrow(T a)(x)=f\left(a^{*} x\right)
\end{aligned}
$$

Como $A^{* *}$ é uma álgebra de von Neumann, segue, pelo Teorema 4.3.13, que $A^{*}$ é $w$-sequencialmente completo. Então, pelo Teorema 4.3.14, $T$ é $w$-compacto.

Pelo Teorema 2.1.2 (c), como $A$ tem a propriedade DP, então $T x_{n} \longrightarrow 0$. Como $x_{n} \stackrel{w}{\longrightarrow} 0$, segue, pela Proposição 1.1.34, que existe um $\alpha>0$ tal que $\left\|x_{n}\right\| \leq \alpha$.

Dado $\varepsilon>0$, existe $n_{0} \in \mathbb{N}$ tal que $\left\|T x_{n}\right\|<\frac{\varepsilon}{\alpha}$, para todo $n \geq n_{0}$. Assim, para todo $n \geq n_{0}$, temos que

$$
\left|f\left(x_{n}^{*} x_{n}\right)\right|=\left|\left(T x_{n}\right)\left(x_{n}\right)\right| \leq\left\|T x_{n}\right\|\left\|x_{n}\right\|<\frac{\varepsilon}{\alpha} \alpha=\varepsilon .
$$

Logo, $f\left(x_{n}^{*} x_{n}\right) \longrightarrow 0$ e, portanto, $x_{n}^{*} x_{n} \stackrel{w}{\longrightarrow} 0$.

(b) $\Rightarrow$ (c) Seja $x_{n} \stackrel{w}{\longrightarrow} 0$ em $A$. Então, por hipótese, $x_{n}^{*} x_{n} \stackrel{w}{\longrightarrow} 0$. Segue, do Lema 4.4.1, que $x_{n} x_{n}^{*}=\left(x_{n}^{*} x_{n}\right)^{*} \stackrel{w}{\longrightarrow} 0$.

(c) $\Rightarrow$ (d) Seja $x_{n} \stackrel{w}{\longrightarrow} 0$ em $A$. Então, por hipótese, $x_{n} x_{n}^{*} \stackrel{w}{\longrightarrow} 0$. Segue, do Lema 4.4.1, que $x_{n}^{*} x_{n}=\left(x_{n} x_{n}^{*}\right)^{*} \stackrel{w}{\longrightarrow} 0$. Logo, $x_{n}^{*} x_{n}+x_{n} x^{*} x_{n} \stackrel{w}{\longrightarrow} 0$.

(d) $\Rightarrow$ (a) Requer o estudo de outras topologias em espaços localmente convexos que foge ao escopo deste trabalho. Para a demonstração desta implicação, vide [11].

Corolário 4.4.3. Seja A uma $C^{*}$-álgebra. Se A tem a propriedade DP, então toda $C^{*}$-subálgebra $B$ de $A$ tem a propriedade DP.

Demonstração. Seja $b_{n} \stackrel{w}{\longrightarrow} 0$ em $B$. Como $B$ é $C^{*}$-subálgebra, segue que $b_{n} \stackrel{w}{\longrightarrow} 0$ em $A$. Pelo Teorema $4.4 .2, b_{n}^{*} b_{n} \stackrel{w}{\longrightarrow} 0$ em $A$. Mas $\left(b_{n}^{*} b_{n}\right)_{n} \subset B$, então $b_{n}^{*} b_{n} \stackrel{w}{\longrightarrow} 0$ em $B$. Logo, $B$ tem a propriedade DP.

Vejamos um resultado análogo para a propriedade DP1.

Teorema 4.4.4. Seja A uma $C^{*}$-álgebra. As seguintes condições são equivalentes:

(a) A tem a propriedadae DP1.

(b) Se $x_{n} \stackrel{w}{\longrightarrow} x$ em $A$, com $\left\|x_{n}\right\|=\|x\|=1$, então $x_{n}^{*} x_{n} \stackrel{w}{\longrightarrow} x^{*} x$;

(c) Se $x_{n} \stackrel{w}{\longrightarrow} x$ em $A$, com $\left\|x_{n}\right\|=\|x\|=1$, então $x_{n} x_{n}^{*} \stackrel{w}{\longrightarrow} x x^{*}$;

(d) Se $x_{n} \stackrel{w}{\longrightarrow} x$ em $A$, com $\left\|x_{n}\right\|=\|x\|=1$, então $x_{n}^{*} x_{n}+x_{n} x_{n}^{*} \stackrel{w}{\longrightarrow} x^{*} x+x x^{*}$. 
Demonstração. (a) $\Rightarrow$ (b) Sejam $x_{n} \stackrel{w}{\longrightarrow} x$ em $A$, com $\left\|x_{n}\right\|=\|x\|=1$, e $g \in A^{*}$. Definimos $f_{n}=g x_{n}^{*} \in A^{*}$, para todo $n \in \mathbb{N}$, e $f=g x^{*} \in A^{*}$, em que $f_{n}(a)=g x_{n}^{*}(a)=g\left(x_{n}^{*} a\right)$ e $f(a)=$ $g x^{*}(a)=g\left(x^{*} a\right)$, para todo $a \in A$.

Por meio da aplicação canônica, podemos identificar os elementos de $A$ com elementos de $A^{* *}$ e, da mesma maneira, os elementos de $A^{*}$ com elementos de $A^{* * *}$. Assim, seja $z \in A^{* *}$, temos que

$$
\begin{aligned}
f_{n}(z) & =g x_{n}^{*}(z)=g\left(x_{n}^{*} z\right)=z g\left(x_{n}^{*}\right) \quad \mathrm{e} \\
f(z) & =g x^{*}(z)=g\left(x^{*} z\right)=z g\left(x^{*}\right) .
\end{aligned}
$$

Como $x_{n} \stackrel{w}{\longrightarrow} x$ em $A$, segue, pelo Lema 4.4.1, que $x_{n}^{*} \stackrel{w}{\longrightarrow} x^{*}$ em $A$. Assim, $z g\left(x_{n}^{*}\right) \longrightarrow z g\left(x^{*}\right)$, então $f_{n} \stackrel{w}{\longrightarrow} f$ em $A^{*}$.

Como $A$ tem a propriedade DP1, temos que $f_{n}\left(x_{n}\right) \longrightarrow f(x)$. Mas

$$
\begin{aligned}
g\left(x_{n}^{*} x_{n}\right) & =g x_{n}^{*}\left(x_{n}\right)=f_{n}\left(x_{n}\right) \quad \mathrm{e} \\
g\left(x^{*} x\right) & =g x^{*}(x)=f(x) .
\end{aligned}
$$

Logo, $x_{n}^{*} x_{n} \stackrel{w}{\longrightarrow} x^{*} x$.

(b) $\Rightarrow$ (c) Seja $x_{n} \stackrel{w}{\longrightarrow} x$ em $A$, com $\left\|x_{n}\right\|=\|x\|=1$, tal que $x_{n}^{*} x_{n} \stackrel{w}{\longrightarrow} x^{*} x$. Então, pelo Lema 4.4.1, $x_{n} x_{n}^{*}=\left(x_{n}^{*} x_{n}\right)^{*} \stackrel{w}{\longrightarrow}\left(x^{*} x\right)^{*}=x x^{*}$.

(c) $\Rightarrow$ (d) Seja $x_{n} \stackrel{w}{\longrightarrow} 0$ em $A$, com $\left\|x_{n}\right\|=\|x\|=1$, tal que $x_{n} x_{n}^{*} \stackrel{w}{\longrightarrow} x x^{*}$. Pelo Lema 4.4.1, temos que $x_{n}^{*} x_{n}=\left(x_{n} x_{n}^{*}\right)^{*} \stackrel{w}{\longrightarrow}\left(x x^{*}\right)^{*}=x^{*} x$. Logo, $x_{n}^{*} x_{n}+x_{n} x^{*} x_{n} \stackrel{w}{\longrightarrow} x^{*} x+x x^{*}$.

(d) $\Rightarrow$ (a) Requer o estudo de outras topologias em espaços localmente convexos que foge ao escopo deste trabalho. Para a demonstração desta implicação, vide [21].

Corolário 4.4.5. Seja A uma $C^{*}$-álgebra. Se A tem a propriedade DP1, então toda $C^{*}$-subálgebra $B$ de $A$ tem a propriedade DP1.

Demonstração. Seja $b_{n} \stackrel{w}{\longrightarrow} b$ em $B$, com $\left\|b_{n}\right\|=\|b\|=1$. Como $B$ é $C^{*}$-subálgebra, segue que $b_{n} \stackrel{w}{\longrightarrow} b$ em $A$. Pelo Teorema 4.4.4, $b_{n}^{*} b_{n} \stackrel{w}{\longrightarrow} b^{*} b$ em $A$. Mas $\left(b_{n}^{*} b_{n}\right)_{n} \subset B$, então $b_{n}^{*} b_{n} \stackrel{w}{\longrightarrow} b^{*} b$ em $B$. Logo, $B$ tem a propriedade DP1.

Nosso objetivo, a seguir, é estudar a demonstração de que $K(H)$ não possui a propriedade DP1. Para tanto, um operador compacto análogo ao da Definição 1.1.8 será definido.

Exemplo 4.4.6. Seja $H$ um espaço de Hilbert e $x, y \in H$. Definimos o operador $x \otimes y$ em $H$ por

$$
(x \otimes y)(z)=\langle z, y\rangle x
$$

para todo $z \in H$. Temos que $\|x \otimes y\|=\|x\|\|y\|$ e se $x, y$ são não nulos, então o operador $x \otimes y$ tem 
posto 1. Para $x, y, a, b \in H$, temos que:

$$
\begin{aligned}
(x \otimes a)(y \otimes b) & =\langle y, a\rangle(x \otimes b) \quad \mathrm{e} \\
(x \otimes y)^{*} & =y \otimes x .
\end{aligned}
$$

De fato, para todo $h, z \in H$, temos que

$$
\begin{aligned}
(x \otimes a)(y \otimes b)(h)= & (x \otimes a)(\langle h, b\rangle y)=\langle h, b\rangle(x \otimes a)(y) \\
= & \langle h, b\rangle\langle y, a\rangle x=\langle y, a\rangle\langle h, b\rangle x \\
= & \langle y, a\rangle(x \otimes b)(h), \\
\langle(x \otimes y)(h), z\rangle & =\langle\langle h, y\rangle x, z\rangle=\langle h, y\rangle\langle x, z\rangle \\
& =\overline{\langle z, x\rangle}\langle h, y\rangle=\langle h,\langle z, x\rangle y\rangle \\
& =\langle h,(y \otimes x)(z)\rangle .
\end{aligned}
$$

Logo, $(x \otimes a)(y \otimes b)=\langle y, a\rangle(x \otimes b)$ e $(x \otimes y)^{*}=y \otimes x$.

Proposição 4.4.7. Se $H$ é um espaço de Hilbert de dimensão infinita, então $K(H)$ não tem a propriedade DP1.

Demonstração. Seja $H$ um espaço de Hilbert de dimensão infinita e $\left(e_{n}\right)_{n}$ uma sequência ortonormal em $H$. Vamos mostrar que $K(H)$ não possui a propriedade DP1.

Para cada $n \in \mathbb{N}$, considere $T_{n}=e_{n} \otimes e_{1}+e_{2} \otimes e_{2}$ e $T=e_{2} \otimes e_{2}$. Como $T_{n}$ e $T$ tem posto finito, então $T_{n} \in K(H)$, para todo $n \in \mathbb{N}$, e $T \in K(H)$.

Temos que $\|T\|=\left\|e_{2} \otimes e_{2}\right\|=\left\|e_{2}\right\|\left\|e_{2}\right\|=1 \mathrm{e}$

$$
\begin{aligned}
\left\|T_{n}\right\| & =\left\|e_{n} \otimes e_{1}+e_{2} \otimes e_{2}\right\|=\sup _{x \in B_{H}}\left\|\left(e_{n} \otimes e_{1}+e_{2} \otimes e_{2}\right)(x)\right\| \\
& =\sup _{x \in B_{H}}\left\|\left\langle x, e_{n}\right\rangle e_{1}+\left\langle x, e_{2}\right\rangle e_{2}\right\| \leq \sup _{x \in B_{H}}\left(\left|\left\langle x, e_{n}\right\rangle\right|\left\|e_{1}\right\|+\left|\left\langle x, e_{2}\right\rangle\right|\left\|e_{2}\right\|\right) .
\end{aligned}
$$

Pela desigualdade de Bessel (Teorema 1.3.2), temos que, para todo $x \in B_{H}$,

$$
\left|\left\langle x, e_{n}\right\rangle\right|+\left|\left\langle x, e_{2}\right\rangle\right| \leq\left|\left\langle x, e_{n}\right\rangle\right|^{2}+\left|\left\langle x, e_{2}\right\rangle\right|^{2} \leq \sum_{i=1}^{\infty}\left|\left\langle x, e_{i}\right\rangle\right|^{2} \leq\|x\|^{2} \leq 1 .
$$

Então,

$$
\left\|T_{n}\right\| \leq \sup _{x \in B_{H}}\left(\left|\left\langle x, e_{n}\right\rangle\right|\left\|e_{1}\right\|+\left|\left\langle x, e_{2}\right\rangle\right|\left\|e_{2}\right\|\right) \leq 1
$$

Considerando $x=e_{2}$, temos que

$$
\left\|\left(e_{n} \otimes e_{1}+e_{2} \otimes e_{2}\right)\left(e_{2}\right)\right\|=\left\|\left\langle e_{2}, e_{n}\right\rangle e_{1}+\left\langle e_{2}, e_{2}\right\rangle e_{2}\right\|=\left\|e_{2}\right\|=1 .
$$

Logo, $\left\|T_{n}\right\|=1$.

Vamos mostrar que $T_{n} \stackrel{w}{\longrightarrow} T$ e $T_{n}^{*} T_{n} \stackrel{w}{\leftrightarrow} T^{*} T$, pois, pelo Teorema 4.4.4, seguirá que $K(H)$ não possui a propriedade DP1. 
Primeiramente, mostraremos que $T_{n} \stackrel{w}{\longrightarrow} T$, isto é, $f\left(T_{n}\right) \longrightarrow f(T)$, para todo $f \in K(H)^{*}$.

Seja $f \in K(H)^{*}$. Pelo Teorema 4.1.30, temos que $K(H)^{*} \cong \mathscr{L}_{1}(H)$. Então, existe $S \in \mathscr{L}_{1}(H)$ tal que $f=\Phi_{S}$, isto é, $f(U)=\Phi_{S}(U)=\operatorname{tr}(U S)$, para todo $U \in K(H)$. Assim,

$$
\begin{aligned}
\left|f\left(T_{n}\right)-f(T)\right| & =\left|f\left(e_{n} \otimes e_{1}+e_{2} \otimes e_{2}-e_{2} \otimes e_{2}\right)\right|=\left|f\left(e_{n} \otimes e_{1}\right)\right|=\left|\Phi_{S}\left(e_{n} \otimes e_{1}\right)\right| \\
& =\left|\operatorname{tr}\left(\left(e_{n} \otimes e_{1}\right) S\right)\right|=\left|\sum_{i=1}^{\infty}\left\langle\left(e_{n} \otimes e_{1}\right) S\left(e_{i}\right), e_{i}\right\rangle\right|=\left|\sum_{i=1}^{\infty}\left\langle\left\langle S\left(e_{i}\right), e_{n}\right\rangle e_{1}, e_{i}\right\rangle\right| \\
& =\left|\sum_{i=1}^{\infty}\left\langle S\left(e_{i}\right), e_{n}\right\rangle\left\langle e_{1}, e_{i}\right\rangle\right|=\left|\left\langle S\left(e_{1}\right), e_{n}\right\rangle\right| \longrightarrow 0,
\end{aligned}
$$

pois, pela desigualdade de Bessel (Teorema 1.3.2), temos que

$$
\sum_{n=1}^{\infty}\left|\left\langle S\left(e_{1}\right), e_{n}\right\rangle\right|^{2} \leq\left\|S\left(e_{1}\right)\right\|^{2} .
$$

Então a série $\sum_{n=1}^{\infty}\left|\left\langle S\left(e_{1}\right), e_{n}\right\rangle\right|^{2}$ converge, donde $\left|\left\langle S\left(e_{1}\right), e_{n}\right\rangle\right|^{2} \longrightarrow 0$. Logo, $f\left(T_{n}\right) \longrightarrow f(T)$, para todo $f \in K(H)^{*}$, e, portanto, $T_{n} \stackrel{w}{\longrightarrow} T$.

Para $n \geq 3$, vejamos que $T_{n}^{*} T_{n} \stackrel{w}{\leftrightarrow} T^{*} T$, isto é, $f\left(T_{n}^{*} T_{n}\right) \nrightarrow f\left(T^{*} T\right)$, para todo $f \in K(H)^{*}$. Haja vista as propriedades no Exemplo 4.4.6, temos que

$$
\begin{aligned}
T_{n}^{*} T_{n} & =\left(e_{n} \otimes e_{1}+e_{2} \otimes e_{2}\right)^{*}\left(e_{n} \otimes e_{1}+e_{2} \otimes e_{2}\right)=\left(e_{1} \otimes e_{n}+e_{2} \otimes e_{2}\right)\left(e_{n} \otimes e_{1}+e_{2} \otimes e_{2}\right) \\
& =\left(e_{1} \otimes e_{n}\right)\left(e_{n} \otimes e_{1}\right)+\left(e_{1} \otimes e_{n}\right)\left(e_{2} \otimes e_{2}\right)+\left(e_{2} \otimes e_{2}\right)\left(e_{n} \otimes e_{1}\right)+\left(e_{2} \otimes e_{2}\right)\left(e_{2} \otimes e_{2}\right) \\
& =\left\langle e_{n}, e_{n}\right\rangle\left(e_{1} \otimes e_{1}\right)+\left\langle e_{2}, e_{n}\right\rangle\left(e_{1} \otimes e_{2}\right)+\left\langle e_{n}, e_{2}\right\rangle\left(e_{2} \otimes e_{1}\right)+\left\langle e_{2}, e_{2}\right\rangle\left(e_{2} \otimes e_{2}\right) \\
& =e_{1} \otimes e_{1}+e_{2} \otimes e_{2} \\
T^{*} T & =\left(e_{2} \otimes e_{2}\right)^{*}\left(e_{2} \otimes e_{2}\right)=\left(e_{2} \otimes e_{2}\right)\left(e_{2} \otimes e_{2}\right)=e_{2} \otimes e_{2} .
\end{aligned}
$$

Seja $f \in K(H)^{*}$. Pelo Teorema 4.1.30, temos que $K(H)^{*} \cong \mathscr{L}_{1}(H)$. Então, existe $S \in \mathscr{L}_{1}(H)$ tal que $f=\Phi_{S}$, isto é, $f(U)=\Phi_{S}(U)=\operatorname{tr}(U S)$, para todo $U \in K(H)$. Assim,

$$
\begin{aligned}
\left|f\left(T_{n}^{*} T_{n}\right)-f\left(T^{*} T\right)\right| & =\left|f\left(e_{1} \otimes e_{1}+e_{2} \otimes e_{2}-e_{2} \otimes e_{2}\right)\right|=\left|f\left(e_{1} \otimes e_{1}\right)\right|=\left|\Phi_{S}\left(e_{1} \otimes e_{1}\right)\right| \\
& =\left|\operatorname{tr}\left(\left(e_{1} \otimes e_{1}\right) S\right)\right|=\left|\sum_{i=1}^{\infty}\left\langle\left(e_{1} \otimes e_{1}\right) S\left(e_{i}\right), e_{i}\right\rangle\right|=\left|\sum_{i=1}^{\infty}\left\langle\left\langle S\left(e_{i}\right), e_{1}\right\rangle e_{1}, e_{i}\right\rangle\right| \\
& =\left|\sum_{i=1}^{\infty}\left\langle S\left(e_{i}\right), e_{1}\right\rangle\left\langle e_{1}, e_{i}\right\rangle\right|=\left|\left\langle S\left(e_{1}\right), e_{1}\right\rangle\right| \nrightarrow 0 .
\end{aligned}
$$

Logo, $T_{n} \stackrel{w}{\longrightarrow} T$ e $T_{n}^{*} T_{n} \stackrel{w}{\rightarrow} T^{*} T$. Pelo Teorema 4.4.4, segue que $K(H)$ não possui a propriedade DP1.

Decorre da proposição anterior o seguinte exemplo:

Exemplo 4.4.8. Como $K(H)$ é uma $\mathrm{C}^{*}$-subálgebra de $L(H)$ que não tem a propriedade DP1, segue, do Corolário 4.4.5, que $L(H)$ não tem a propriedade DP1. 
O espaço $K(H)$ não tem a propriedade DP, pois não tem a propriedade DP1.

Esse exemplo nos leva ao próximo:

Exemplo 4.4.9. A álgebra de Fermion $\otimes M_{2}$ definida em 4.2 .10 não tem a propriedade DP.

Do último exemplo, $K\left(\ell_{2}\right)$ não tem a propriedade DP. Mas vimos no Teorema 4.2.11 que a álgebra de Fermion $\otimes M_{2}$ contém uma cópia complementada de $K\left(\ell_{2}\right)$ e, pela Proposição 2.1.7, a propriedade DP é preservada por subespaço complementado. Assim, $\otimes M_{2}$ não tem a propriedade DP.

As próximas duas proposições mostram o comportamento da propriedade DP em relação a $*$-homomorfismos.

Proposição 4.4.10. Sejam $A$ uma $C^{*}$-álgebra separável, $I$ um ideal fechado em $A$ e $B$ uma $C^{*}$ álgebra tais que $B$ é isomorfo a $A / I$. Se A tem a propriedade DP, então B também tem a propriedade $D P$.

Demonstração. Seja $b_{n} \stackrel{w}{\longrightarrow} 0$ em $B$. Considere $\pi: A \longrightarrow B$ a aplicação quociente, isto é, a composição do isomorfismo de $A / I$ em $B$ com a aplicação quociente de $A$ em $A / I$. Pela Proposição 4.1.21, existe $\left(a_{n}\right)_{n}$ uma sequência em $A$ tal que $\pi\left(a_{n}\right)=b_{n}$ e $a_{n} \stackrel{w}{\longrightarrow} 0$ em $A$.

Como $A$ tem a propriedade DP, pelo Teorema 4.4.2, temos que $a_{n}^{*} a_{n} \stackrel{w}{\longrightarrow} 0$ em $A$. Mas,

$$
\pi\left(a_{n}^{*} a_{n}\right)=\pi\left(a_{n}^{*}\right) \pi\left(a_{n}\right)=\pi\left(a_{n}\right)^{*} \pi\left(a_{n}\right)=b_{n}^{*} b_{n} \stackrel{w}{\longrightarrow} 0 \quad \text { em } B .
$$

Logo, $B$ tem a propriedade DP.

Proposição 4.4.11. Sejam $A$ uma $C^{*}$-álgebra e $\varphi: A \longrightarrow \varphi(A)$ um *-homomorfismo. Se $A$ tem a propriedade $D P$, então $\varphi(A)$ também tem a propriedade $D P$.

Demonstração. Seja $\left(x_{n}\right)_{n}$ uma sequência em $\varphi(A)$ tal que $x_{n} \stackrel{w}{\longrightarrow} 0$ em $\varphi(A)$. Mostraremos que $x_{n}^{*} x_{n} \stackrel{w}{\longrightarrow} 0$ em $\varphi(A)$.

Sejam $\left(a_{n}\right)_{n}$ uma sequência em $A$ tal que $\varphi\left(a_{n}\right)=x_{n}$ e $B=\left[a_{n}: n \in \mathbb{N}\right]$ uma $C^{*}$-subálgebra de $A$. Como $B$ é gerado por um número enumerável de elementos, então $B$ é separável. Além disso, $\left\{x_{n}: n \in \mathbb{N}\right\} \subset \varphi(B)$ e $x_{n} \stackrel{w}{\longrightarrow} 0$ em $\varphi(B)$.

Consideremos a restrição de $\varphi$ em $B$, isto é, $\varphi \uparrow_{B}: B \longrightarrow \varphi(B)$. Então, pela Proposição 4.1.21, existe $\left(y_{n}\right)_{n}$ uma sequência em $B$ tal que $\varphi\left(y_{n}\right)=x_{n}$ e $y_{n} \stackrel{w}{\longrightarrow} 0$ em $B$.

Em particular, $y_{n} \stackrel{w}{\longrightarrow} 0$ em $A$ e como $A$ tem a propriedade DP, pelo Teorema 4.4.2, temos que $y_{n}^{*} y_{n} \stackrel{w}{\longrightarrow} 0$ em $A$. Mas,

$$
\varphi\left(y_{n}^{*} y_{n}\right)=\varphi\left(y_{n}^{*}\right) \varphi\left(y_{n}\right)=\varphi\left(y_{n}\right)^{*} \varphi\left(y_{n}\right)=x_{n}^{*} x_{n} \stackrel{w}{\longrightarrow} 0 \quad \text { em } \varphi(A) .
$$

Logo, $\varphi(A)$ tem a propriedade DP.

Proposição 4.4.12. Seja A uma $C^{*}$-álgebra. Se toda representação irredutível de A tem dimensão finita, então $A^{*}$, o dual de $A$, possui a propriedade DP.

(vide [23]) 
O teorema a seguir é parte fundamental da equivalência entre as propriedades DP e DP1. A demonstração usa a proposição anterior e muitos dos resultados das seções anteriores.

Teorema 4.4.13. Seja A uma $C^{*}$-álgebra. Então, A tem a propriedade DP se, e somente se, toda representação irredutivel de A tem dimensão finita.

Demonstração. $(\Rightarrow)$ Suponhamos que $A$ tem a propriedade DP. Vamos mostrar que $A$ é do tipo I.

Suponhamos, por absurdo, que $A$ não é do tipo I. Então, pelo Corolário 4.2.13, existem $B$ uma $C^{*}$-subálgebra de $A$ e $J$ um ideal fechado em $B$ tais que $B / J$ é isomorfo à álgebra de Fermion $\otimes M_{2}$. Como $A$ tem a propriedade DP, então, pelo Corolário 4.4.3, $B$ tem a propriedade DP. Como $B$ tem a propriedade DP, segue, pela Proposição 4.4.11, que $B / J$ tem a propriedade DP. Mas, $B / J$ é isomorfo a $\otimes M_{2}$, então $\otimes M_{2}$ tem a propriedade DP. Absurdo, pois, pelo Exemplo 4.4.9, $\otimes M_{2}$ não tem a propriedade DP. Logo, $A$ é do tipo I.

Suponhamos, por absurdo, que $A$ possui uma representação irredutível que tem dimensão infinita, digamos $(\pi, H)$. Como $\pi$ é um $*$-homomorfismo e $A$ tem a propriedade DP, então, pela Proposição 4.4.11, $\pi(A)$ tem a propriedade DP. Como $A$ é do tipo I, segue, pelo Teorema 4.2.8, que $K(H) \subset \pi(A)$. Então, pelo Corolário 4.4.5, $K(H)$ tem a propriedade DP. Absurdo, pois mostramos na Proposição 4.4.7 que se $H$ tem dimensão infinita, então $K(H)$ não tem a propriedade DP.

$(\Leftarrow)$ Suponhamos que toda representação irredutível de $A$ tem dimensão finita, então, segue da Proposição 4.4.12, que $A^{*}$ tem a propriedade DP. Na Seção 2.1, vimos que se o dual de um espaço de Banach $X^{*}$ tem a propriedade DP, então $X$ tem a propriedade DP (Corolário 2.1.8). Assim, como $A^{*}$ tem a propriedade DP, então $A$ tem a propriedade DP.

Nosso objetivo agora é mostrar que se uma $C^{*}$-álgebra $A$ possui a propriedade DP1, então toda representação irredutível de $A$ tem dimensão finita. Para tanto alguns resultados preliminares:

Lema 4.4.14. Seja A uma $C^{*}$-álgebra. Se p é uma projeção em $A^{* *}$, então a $C^{*}$-subálgebra $I:=$ $A \cap p A^{* *} p$ é hereditária de $A^{* *}$.

Demonstração. Consideremos $C$ a aplicação canônica de $A$ em $A^{* *}$. Notemos que em $A \cap p A^{* *} p$ identificamos $A$ com $C(A)$. Assim, $I=A \cap p A^{* *} p=\{x \in C(A): p x p=x\}$. De fato, tome $y \in A \cap p A^{* *} p$, então $y \in C(A)$ e $y \in p A^{* *} p$, isto é, existe $z \in A^{* *}$ tal que $y=p z p$. Mas

$$
y=p z p \Longrightarrow p y p=p p z p p=p z p=y
$$

Tomemos $y \in C(A)$ tal que $y=p y p$, então $y \in A \cap p A^{* *} p$.

Se $I=0$, não há nada a provar. Suponhamos que $I \neq 0$. Sejam $b \in I$ e $a \in A^{* *}$ tais que $0 \leq a \leq b$. Como $b \in I$, segue que $p b p=b$. Temos que $p$ é projeção e é positivo, então $0 \leq p \leq\|p\| \leq 1$. Logo, $0 \leq 1-p$. Assim,

$$
0 \leq(1-p) a(1-p) \leq(1-p) p b p(1-p)
$$

Temos ainda que

$$
(1-p) p b p(1-p)=\left(p b p-p^{2} b p\right)(1-p)=0(1-p)=0 .
$$


Então, $(1-p) a(1-p)=0$. Como

$C^{*}$-ÁLGEBRAS 83

$$
\left\|a^{\frac{1}{2}}(1-p)\right\|^{2}=\left\|\left(a^{\frac{1}{2}}(1-p)\right)^{*} a^{\frac{1}{2}}(1-p)\right\|=\left\|(1-p)^{*}\left(a^{\frac{1}{2}}\right)^{*} a^{\frac{1}{2}}(1-p)\right\|=\|(1-p) a(1-p)\|=0
$$

temos que

$$
a^{\frac{1}{2}}(1-p)=0 \Rightarrow a(1-p)=0 \Rightarrow a=a p \Rightarrow p a=p a p .
$$

Mas, $a=a^{*}=(a p)^{*}=p^{*} a^{*}=p a$, então $a=p a=p a p \in I$. Logo, $I$ é hereditária em $A^{* *}$.

Teorema 4.4.15 (Sakai). Seja $A$ uma $C^{*}$-álgebra tal que o pré-dual de A é um espaço de Banach. Se $a \in A$, então as aplicações de $A$ em $A$, dadas por $x \mapsto a x, x \mapsto x a$ e $x \mapsto x^{*}$, levam sequências $w^{*}$-convergentes em sequências $w^{*}$-convergentes.

(vide [30, p. 18])

Proposição 4.4.16. Sejam $A$ uma $C^{*}$-álgebra e p é uma projeção em $A^{* *}$. Se $I:=A \cap p A^{* *} p$, então $I^{* *}$ é $C^{*}$-subálgebra hereditária de $A^{* *}$.

Demonstração. Mostraremos que $I^{* *} A^{* *} I^{* *} \subseteq I^{* *}$, então, pelo Teorema 4.1.27, seguirá que $I^{* *}$ é hereditária em $A^{* *}$.

Analogamente ao que já fizemos, utilizaremos a aplicação canônica $C$ de $A$ em $A^{* *}$ para identificar a $C^{*}$-álgebra $A$ com $C(A)$. Nesse caso, como visto no Teorema 4.1.11, $A$ é uma $C^{*}$-subálgebra de $A^{* *}$. Aqui, trabalharemos com as $C^{*}$-álgebras $I$ e $A$ e, apesar de não escrevermos, veremos $I$ e $A$ como $C^{*}$-subálgebras do seus respectivos biduais.

Pelo Lema 4.4.14, $I$ é uma $C^{*}$-subálgebra hereditária de $A^{* *}$. Então, pelo Teorema 4.1.27, para todo $a, b \in I$ e todo $z \in A^{* *}$, temos que $a z b \in I$, isto é, $I A^{* *} I \subseteq I$.

Sejam $z \in A^{* *}$ e $a, b \in I$. Pelo Teorema de Goldstine (Teorema 1.1.42), existe uma rede limitada $\left(z_{\lambda}\right)$ em $A$ tal que $\left(z_{\lambda}\right)$ é $w^{*}$-convergente para $z$ em $A^{* *}$. Como $a z_{\lambda} b \in I A^{* *} I \subseteq I$, então, pelo Teorema de Sakai (Teorema 4.4.15), $a z b=w^{*}-\lim _{\lambda} a z_{\lambda} b \in \bar{I}^{w^{*}}$. Pelo Corolário 1.1.43, temos que $a z b \in I^{* *}$. Logo, $I A^{* *} I \subseteq I^{* *}$.

Suponhamos que $z \in A^{* *}, a \in I^{* *}$ e $b \in I$. Como feito acima, existe uma rede limitada $\left(a_{\lambda}\right)$ em $I$ tal que $\left(a_{\lambda}\right)$ é $w^{*}$-convergente para $a$ em $I^{* *}$. Como $a_{\lambda} z b \in I A^{* *} I \subseteq I$, então $a z b=w^{*}-\lim _{\lambda} a_{\lambda} z b \in$ $\bar{I}^{w^{*}}=I^{* *}$. Logo, $I^{* *} A^{* *} I \subseteq I^{* *}$.

Analogamente, suponhamos que $z \in A^{* *}$ e $a, b \in I^{* *}$. Como acima, existe uma rede limitada $\left(b_{\lambda}\right)$ em $I$ tal que $\left(b_{\lambda}\right)$ é $w^{*}$-convergente para $b$ em $I^{* *}$. Como $a z b_{\lambda} \in I^{* *} A^{* *} I \subseteq I^{* *}$, então $a z b=$ $w^{*}-\lim _{\lambda} a z b_{\lambda} \in \overline{I^{* *} w^{*}}=I^{* *}$, pois $\overline{I^{* *} w^{*}}=\overline{\bar{I}^{w^{*}} w^{*}}=\bar{I}^{w^{*}}=I^{* *}$. Assim, $I^{* *} A^{* *} I^{* *} \subseteq I^{* *}$.

Logo, pelo Teorema 4.1.27, segue que $I^{* *}$ é uma $C^{*}$-subálgebra hereditária de $A^{* *}$.

As próximas duas proposições serão fundamentais para a demonstração de que se uma $C^{*}$ álgebra $A$ tem a propriedade DP1, então toda representação irredutível de $A$ tem dimensão finita. A primeira proposição nos dá as hipóteses necessárias para que a $C^{*}$-subálgebra $I$, estudada nos últimos resultados, tenha a propriedade DP. É importante lembrar que $\mathcal{R}(x)$ é a projeção imagem de um elemento $x$ de uma $C^{*}$-álgebra (Definição 4.3.7).

Proposição 4.4.17. Sejam A uma $C^{*}$-álgebra com a propriedade DP1, $x$ um elemento positivo de A não nulo e $p=1-\mathcal{R}(x)$, sendo $\mathcal{R}(x)$ a projeção imagem de $x$ em $A^{* *}$. Então, $I:=A \cap p A^{* *} p$ tem a propriedade DP. 
Demonstração. Sejam $x_{n} \stackrel{w}{\longrightarrow} 0$ em $I$ e $f_{n} \stackrel{w}{\longrightarrow} 0$ em $I^{*}$. Pelo Teorema 2.1.2 (e), basta mostrar que $f_{n}\left(x_{n}\right) \longrightarrow 0$.

Suponhamos que $\|x\|=1$ e $\left\|x_{n}\right\| \leq 1$, para todo $n \in \mathbb{N}$.

Pelo Teorema 4.3.6, toda álgebra de von Neumann possui unidade. Então, consideremos $u$ a unidade da álgebra de von Neumann $I^{* *}$. Como $I^{* *}$ é $C^{*}$-subálgebra de $A^{* *}$, então $u$ é projeção de $A^{* *}$ e $u b u \in A^{* *}$, para todo $b \in I^{* *}$. Assim, $I^{* *} \subset u A^{* *} u$. Pela Proposição 4.4.16, temos que $I^{* *} A^{* *} I^{* *} \subseteq I^{* *}$, então $u A^{* *} u \subset I^{* *}$. Logo, $I^{* *}=u A^{* *} u$.

Para cada $n \in \mathbb{N}$, definimos $\varphi\left(g_{n}\right):=(u \varphi u)\left(f_{n}\right)$, para todo $\varphi \in A^{* *}$. Em particular, definimos $g_{n}(a)=C(a)\left(g_{n}\right)=(u C(a) u)\left(f_{n}\right)$, em que $C$ é a aplicação canônica de $A$ em $A^{* *}$. Assim, $g_{n} \in A^{*}$.

Vejamos que $g_{n} \stackrel{w}{\longrightarrow} 0 \mathrm{em} A^{*}$. Seja $\varphi \in A^{* *}$. Como $I^{* *}=u A^{* *} u$, então $q \varphi q \in I^{* *}$. Além disso, $f_{n} \stackrel{w}{\longrightarrow} 0$ em $I^{* *}$, então $\varphi\left(g_{n}\right)=(u \varphi u)\left(f_{n}\right) \longrightarrow 0$. Assim, $g_{n} \stackrel{w}{\longrightarrow} 0$ em $A^{*}$.

Como $x_{n} \stackrel{w}{\longrightarrow} 0$, segue que $x_{n}+x \stackrel{w}{\longrightarrow} x$ em $A$. Além disso, pela Observação 4.3 .8 , temos que

$$
\begin{gathered}
p x=(1-\mathcal{R}(x)) x=x-\mathcal{R}(x) x=x-x=0 \quad \mathrm{e} \\
x p=x(1-\mathcal{R}(x))=x-x \mathcal{R}(x)=x-x=0,
\end{gathered}
$$

e como $I=\{y \in C(A): y=p y p\}$ e $\left(x_{n}\right) \subset I$, então $x_{n}=p x_{n} p$.

Para todo $n \in \mathbb{N}$, temos que

$$
\begin{array}{r}
x_{n}^{*} x=\left(p x_{n} p\right)^{*} x=p x_{n}^{*} p x=0, \\
x^{*} x_{n}=x^{*}\left(p x_{n} p\right)=x p x_{n} p=0, \\
x_{n}^{*} x_{n} x^{*} x=p x_{n}^{*} p p x_{n} p x^{*} x=0, \\
x^{*} x x_{n}^{*} x_{n}=x^{*} x p x_{n}^{*} p p x_{n} p=0, \\
\left(x_{n}^{*} x_{n}\right)^{*} x^{*} x=p x_{n}^{*} p p x_{n} p x^{*} x=0 \quad \mathrm{e} \\
x^{*} x\left(x_{n}^{*} x_{n}\right)^{*}=x^{*} x p x_{n}^{*} p p x_{n} p=0 .
\end{array}
$$

Pela definição de elemento ortogonal (Definição 4.1.18), segue que $x_{n}^{*} x_{n} \perp x^{*} x$, para todo $n \in \mathbb{N}$. Pela definicão de $C^{*}$-álgebra (Definição 4.1.6) e pela Proposição 4.1.19, temos que

$$
\begin{aligned}
\left\|x_{n}+x\right\|^{2} & =\left\|\left(x_{n}+x\right)^{*}\left(x_{n}+x\right)\right\|=\left\|x_{n}^{*} x_{n}+x_{n}^{*} x+x^{*} x_{n}+x^{*} x\right\|=\left\|x_{n}^{*} x_{n}+x^{*} x\right\| \\
& =\max \left\{\left\|x_{n}^{*} x_{n}\right\|,\left\|x^{*} x\right\|\right\}=\max \left\{\left\|x_{n}\right\|^{2},\|x\|^{2}\right\}=1 .
\end{aligned}
$$

Assim, $x_{n}+x \stackrel{w}{\longrightarrow} x$ em $A$, com $\left\|x_{n}+x\right\|=\|x\|=1$, e $g_{n} \stackrel{w}{\longrightarrow} 0$ em $A^{* *}$. Como $A$ tem a propriedade DP1, segue que $g_{n}\left(x_{n}+x\right) \longrightarrow 0$ em $A$. Mas, $g_{n} \stackrel{w}{\longrightarrow} 0$ em $A^{*}$, então $g_{n}(x) \longrightarrow 0$. Portanto, $g_{n}\left(x_{n}\right) \longrightarrow 0$.

Como $C\left(x_{n}\right) \subset I^{* *}$ e lembrando que $u$ é unidade de $I^{* *}$, temos que $g_{n}\left(x_{n}\right)=\left(u C\left(x_{n}\right) u\right)\left(f_{n}\right)=$ $C\left(x_{n}\right)\left(f_{n}\right)=f_{n}\left(x_{n}\right)$.

Logo, $f_{n}\left(x_{n}\right) \longrightarrow 0$ e, portanto, $I$ tem a propriedade DP.

Utilizando o operador definido no Exemplo 4.4.6, obtemos a seguinte proposição.

Proposição 4.4.18. Se $H$ é um espaço de Hilbert de dimensão infinita, então existem $U$ e $V$ operadores em $H$ compactos, positivos, ortogonais, $U(H)$ e $V(H)$ têm dimensão infinita. 
Demonstração. Seja $\left(e_{n}\right)_{n}$ uma sequência ortonormal em $H$. Consideremos $U, V: H \longrightarrow H$, dados por

$$
U=\sum_{n=1}^{\infty} \frac{1}{n} e_{2 n} \otimes e_{2 n} \quad \text { e } \quad V=\sum_{n=1}^{\infty} \frac{1}{n} e_{2 n+1} \otimes e_{2 n+1} .
$$

Mostraremos que $U$ e $V$ são compactos, positivos, ortogonais, $U(H)$ e $V(H)$ têm dimensão infinita. Usaremos a definição e as propriedades vistas no Exemplo 4.4.6.

Pela definição dos operadores $U$ e $V$, para todo $x \in H$, temos que

$$
\begin{aligned}
& U(x)=\left(\sum_{n=1}^{\infty} \frac{1}{n} e_{2 n} \otimes e_{2 n}\right)(x)=\sum_{n=1}^{\infty} \frac{1}{n}\left\langle x, e_{2 n}\right\rangle e_{2 n}=\left(0, x_{2}, 0, \frac{1}{2} x_{4}, 0, \frac{1}{3} x_{6}, \ldots\right) \text { e } \\
& V(x)=\left(\sum_{n=1}^{\infty} \frac{1}{n} e_{2 n+1} \otimes e_{2 n+1}\right)(x)=\sum_{n=1}^{\infty} \frac{1}{n}\left\langle x, e_{2 n+1}\right\rangle e_{2 n+1}=\left(x_{1}, 0, \frac{1}{2} x_{3}, 0, \frac{1}{3} x_{5}, \ldots\right) .
\end{aligned}
$$

É fácil ver que $U$ e $V$ têm imagem de dimensão infinita.

Segue também do Exemplo 4.4.6 que $\left(e_{2 i} \otimes e_{2 i}\right)\left(e_{2 j+1} \otimes e_{2 j+1}\right)=\left\langle e_{2 j+1}, e_{2 i}\right\rangle\left(e_{2 i} \otimes e_{2 j+1}\right)=0$, para todo $i, j=1,2, \ldots$ Assim, $U V=V U=0$, então $U$ e $V$ são ortogonais.

Pela Definição 1.3.7, $U$ e $V$ são positivos se $\langle U(x), x\rangle \geq 0$ e $\langle V(x), x\rangle \geq 0$, para todo $x \in H$. Seja $x \in H$, então

$$
\begin{aligned}
& \langle U(x), x\rangle=\left\langle\left(\sum_{n=1}^{\infty} \frac{1}{n} e_{2 n} \otimes e_{2 n}\right)(x), x\right\rangle=\sum_{n=1}^{\infty} \frac{1}{n}\left\langle x, e_{2 n}\right\rangle\left\langle e_{2 n}, x\right\rangle=\sum_{n=1}^{\infty} \frac{1}{n}\left|\left\langle x, e_{2 n}\right\rangle\right|^{2} \geq 0 \\
& \langle V(x), x\rangle=\left\langle\left(\sum_{n=1}^{\infty} \frac{1}{n} e_{2 n+1} \otimes e_{2 n+1}\right)(x), x\right\rangle=\sum_{n=1}^{\infty} \frac{1}{n}\left\langle x, e_{2 n+1}\right\rangle\left\langle e_{2 n+1}, x\right\rangle=\sum_{n=1}^{\infty} \frac{1}{n}\left|\left\langle x, e_{2 n+1}\right\rangle\right|^{2} \geq 0 .
\end{aligned}
$$

Logo, $U$ e $V$ são positivos.

Por último, vejamos que $U$ e $V$ são compactos. Considere $U_{k}=\sum_{n=1}^{k} \frac{1}{n} e_{2 n} \otimes e_{2 n}$. Para todo $k \in \mathbb{N}$, $U_{k}$ é compacto, pois $U_{k}$ tem posto finito. Vamos mostrar que $U_{k} \stackrel{n=1}{\longrightarrow} U$, então, pela Proposição 1.1.18, seguirá que $U$ é compacto.

Seja $x \in H$ tal que $\|x\|=1$ e $x=\left(x_{1}, x_{2}, \ldots, x_{n}, \ldots\right)$. Então,

$$
\begin{aligned}
\left\|\left(U-U_{k}\right)(x)\right\|^{2} & =\left\|\left(\sum_{n=k+1}^{\infty} \frac{1}{n} e_{2 n} \otimes e_{2 n}\right)(x)\right\|^{2}=\left\|\sum_{n=k+1}^{\infty} \frac{1}{n}\left\langle x, e_{2 n}\right\rangle e_{2 n}\right\|^{2} \\
& =\left\langle\sum_{n=k+1}^{\infty} \frac{1}{n}\left\langle x, e_{2 n}\right\rangle e_{2 n}, \sum_{n=k+1}^{\infty} \frac{1}{n}\left\langle x, e_{2 n}\right\rangle e_{2 n}\right\rangle=\sum_{n=k+1}^{\infty} \frac{1}{n^{2}}\left|x_{2 n}\right|^{2} \\
& \leq \frac{1}{(k+1)^{2}} \sum_{n=k+1}^{\infty}\left|x_{2 n}\right|^{2} \leq \frac{\|x\|^{2}}{(k+1)^{2}}=\frac{1}{(k+1)^{2}} .
\end{aligned}
$$

Assim,

$$
\left\|U-U_{k}\right\|=\sup _{\|x\|=1}\left\|\left(U-U_{k}\right)(x)\right\| \leq \frac{1}{(k+1)} .
$$


Dado $\varepsilon>0$, tome $k_{0}=\frac{1}{\varepsilon}$. Então, para todo $k \geq k_{0}$, temos que

$$
\left\|U-U_{k}\right\| \leq \frac{1}{k+1}<\frac{1}{k_{0}}=\varepsilon
$$

Analogamente, se considerarmos $V_{k}=\sum_{n=1}^{k} \frac{1}{n} e_{2 n+1} \otimes e_{2 n+1}$, temos que, para todo $k \in \mathbb{N}, V_{k}$ é compacto, pois $V_{k}$ tem posto finito e $V_{k} \longrightarrow V$, então $V$ é compacto.

Teorema 4.4.19. Seja $(\pi, H)$ uma representação irredutivel de $A$. Se $P_{1}$ e $P_{2}$ são projeções de posto 1 em $L(H)$, então existem dois elementos ortogonais, positivos e não nulos $U$ e $V$ em $\pi(A)$ tais que $P_{1} \leq U$ e $P_{2} \leq V$.

(vide [20])

Teorema 4.4.20. Seja A uma $C^{*}$-álgebra. Se A tem a propriedade DP1, então toda representação irredutivel de A tem dimensão finita.

Demonstração. Seja $(\pi, H)$ uma representação irredutível de $A$. Mostraremos que $H$ tem dimensão finita. Podemos supor que $H$ tem dimensão maior do que um, caso contrário não há o que ser feito.

Como $\operatorname{dim}(H) \geq 2$, podemos tomar duas projeções ortogonais $P_{1}$ e $P_{2}$ de posto 1 em $L(H)$. Então, pelo Teorema 4.4.19, existem dois elementos ortogonais, positivos e não nulos $U$ e $V$ em $\pi(A)$.

Então, existem $z, w \in A$ tais que $U=\pi(z), V=\pi(w)$ e

$$
0=U V=\pi(z) \pi(w)=\pi(z w) \Rightarrow z w \in \operatorname{Nuc}(\pi)
$$

Como $z, w \in A, z w \in \operatorname{Nuc}(\pi)$ e Nuc $(\pi)$ é um ideal fechado de $A$, então, pela Proposição 4.1.22, existem $a, b \in \operatorname{Nuc}(\pi)$ tais que $x:=(z-a), y:=(w-b) \in A^{+}$e $x y=(z-a)(w-b)=0$. Assim,

$$
\begin{aligned}
& \pi(x)=\pi(z-a)=\pi(z)-\pi(a)=U \quad \mathrm{e} \\
& \pi(y)=\pi(w-b)=\pi(w)-\pi(b)=V .
\end{aligned}
$$

Vejamos que $A(y) \subset A \cap p A^{* *} p$, sendo $A(y):=\overline{y A y}, p=1-\mathcal{R}(y)$ e $\mathcal{R}(y)$ a projeção imagem de $y$. Pelo Corolário 4.1.28, temos que $A(y) \subset A$. Então, basta mostrar que $A(y) \subset p A^{* *} p$, lembrando que consideramos $A(y)$ como $C^{*}$-subálgebra de $A(y)^{* *}$ com o uso da aplicação canônica $C$.

Pela Proposição 4.3.10, temos que $C(A(y)) \subset A(y)^{* *}=\mathcal{R}(y) A^{* *} \mathcal{R}(y)$. Mostraremos que $\mathcal{R}(y) A^{* *} \mathcal{R}(y) \subset$ $p A^{* *} p$.

Seja $\varphi \in A^{* *}$. Então, $\mathcal{R}(y) \varphi \mathcal{R}(y) \in \mathcal{R}(y) A^{* *} \mathcal{R}(y)$. Como $x \perp y$, então $\mathcal{R}(x) \perp \mathcal{R}(y)$ e

$$
\begin{aligned}
p \mathcal{R}(y) \varphi \mathcal{R}(y) p & =(1-\mathcal{R}(x)) \mathcal{R}(y) \varphi \mathcal{R}(y)(1-\mathcal{R}(x)) \\
& =\mathcal{R}(y) \varphi \mathcal{R}(y)-\mathcal{R}(x) \mathcal{R}(y) \varphi \mathcal{R}(y)-\mathcal{R}(y) \varphi \mathcal{R}(y) \mathcal{R}(x)+\mathcal{R}(x) \mathcal{R}(y) \varphi \mathcal{R}(y) \mathcal{R}(x) \\
& =\mathcal{R}(y) \varphi \mathcal{R}(y) .
\end{aligned}
$$

Assim, $\mathcal{R}(y) \varphi \mathcal{R}(y) \in p A^{* *} p$. 
Como $A$ tem a propriedade DP1, segue, pelo Proposição 4.4.17, que $A \cap p A^{* *} p$ tem a propriedade $\mathrm{DP}$, em que $p=1-\mathcal{R}(x)$. Assim, como $A \cap p A^{* *} p$ tem a propriedade DP e $A(y) \subset A \cap p A^{* *} p$, segue, pelo Corolário 4.4.3, que $A(y)$ tem a propriedade DP.

Pelo Corolário 4.1.28, temos que $A(y)$ é uma $C^{*}$-subálgebra hereditária de $A$ e $y \in A(y) \not \subset$ Nuc $\pi$, então, pelo Teorema 4.2.5, temos que $\left(\pi \Gamma_{A(y)}, \pi(A(y))(H)\right)$ é uma representação irredutível de $A(y)$. Mas, pelo Teorema 4.4.13, toda representação irredutível de $A(y)$ tem dimensão finita, pois $A(y)$ tem a propriedade DP. Então, $\pi(A(y))(H)$ tem dimensão finita.

Como $y \in A(y)$, então $\pi(y)(H)=V(H)$ tem dimensão finita e, portanto, $V$ tem posto finito.

Analogamente, se tomarmos $q=1-\mathcal{R}(y)$, então $x \in A(x)$ e, portanto, $U$ tem posto finito.

Como $\pi$ é uma representação irredutível de $A, 0 \neq U \in K(H) \cap \pi(A)$ e $0 \neq V \in K(H) \cap \pi(A)$, segue, pelo Lema 4.2.6, que $K(H) \subset \pi(A)$.

Assim, mostrarmos que, para todos $T, S \in K(H)$ operadores positivos, ortogonais e não nulos, temos que $T, S \in \pi(A)$, pois $K(H) \subset \pi(A)$. Como $T, S \in \pi(A)$ e são positivos, ortogonais e não nulos, segue que $T$ e $S$ têm posto finito. Logo, segue da Proposição 4.4.18, que $H$ tem dimensão finita.

O seguinte teorema resume os últimos resultados demonstrados, evidenciando que as propriedades DP e DP1 são equivalentes em $C^{*}$-álgebras.

Teorema 4.4.21. Seja A uma $C^{*}$-álgebra. São equivalentes:

(a) A tem a propriedade DP1;

(b) Toda representação irredutivel de A tem dimensão finita;

(c) A tem a propriedade DP.

Demonstração. (a) $\Rightarrow$ (b) Segue do Teorema 4.4.20.

(b) $\Leftrightarrow$ (c) Segue do Teorema 4.4.13.

(c) $\Rightarrow$ (a) Segue da Proposição 2.2.4.

Encerramos ao mostrar uma caracterização para as propriedades DP e DP1 em $C^{*}$-subálgebras da álgebra $K(H)$.

Proposição 4.4.22. Seja A uma $C^{*}$-subálgebra de $K(H)$. As seguintes condições são equivalentes:

(a) A tem a propriedade DP1;

(b) Para toda sequência $T_{n} \stackrel{w}{\longrightarrow} 0$ em $A$ tal que existe $T \in S_{A}$ e $\left\|T+T_{n}\right\|=1$, temos que $\left\|T_{n}(h)\right\| \longrightarrow 0$, para todo $h \in H$;

(c) A tem a propriedade DP;

(d) Para toda sequência $\left(T_{n}\right)_{n} \subset A$ tal que $T_{n} \stackrel{w}{\longrightarrow} 0$, temos que $\left\|T_{n}(h)\right\| \longrightarrow 0$, para todo $h \in H$. 
Demonstração. (a) $\Leftrightarrow$ (c) Segue do Teorema 4.4.21.

(a) $\Rightarrow$ (b) Sejam $\left(T_{n}\right)_{n} \subset A$ e $T \in S_{A}$ tais que $T_{n} \stackrel{w}{\longrightarrow} 0$ e $\left\|T_{n}+T\right\|=1$. Como $A$ tem a propriedade DP1, então, pelo Teorema 4.4.4, $T_{n}^{*} T_{n} \stackrel{w}{\longrightarrow} 0$ em $A$. Assim, para todo $\varphi \in A^{*}$, $\varphi\left(T_{n}^{*} T_{n}\right) \longrightarrow 0$.

Em particular, considere $\varphi=f \circ \delta_{h} \in A^{*}$, em que $f \in H^{*}$ e $\delta_{h}: A \longrightarrow H$ é o operador avaliação, para todo $h \in H$. Então, para todo $h \in H$ e todo $f \in H^{*}$, temos que

$$
\varphi\left(T_{n}^{*} T_{n}\right)=\left(f \circ \delta_{h}\right)\left(T_{n}^{*} T_{n}\right)=f\left(T_{n}^{*} T_{n}(h)\right) \longrightarrow 0 .
$$

Para todo $f \in H^{*}$, existe $z \in H$ tal que $f(h)=\langle h, z\rangle$, para todo $h \in H$. Então, para todo $h, z \in H,\left\langle T_{n}^{*} T_{n}(h), z\right\rangle \longrightarrow 0$.

Logo, para todo $h \in H$, temos que

$$
\left\langle T_{n}^{*} T_{n}(h), h\right\rangle \longrightarrow 0 \Rightarrow\left\langle T_{n}(h), T_{n}(h)\right\rangle \longrightarrow 0 \Rightarrow\left\|T_{n}(h)\right\| \longrightarrow 0 .
$$

(b) $\Rightarrow$ (a) Sejam $\left(T_{n}\right)_{n} \subset A$ e $T \in S_{A}$ tais que $T_{n} \stackrel{w}{\longrightarrow} 0$ e $\left\|T_{n}+T\right\|=1$. Por hipótese, $\left\|T_{n}(h)\right\| \longrightarrow 0$, para todo $h \in H$, então $\left\|T_{n}^{*} T_{n}(h)\right\| \leq\left\|T_{n}^{*}\right\|\left\|T_{n}(h)\right\| \longrightarrow 0$, para todo $h \in H$. Assim, para todo $h_{1}, h_{2} \in H,\left\langle T_{n}^{*} T_{n}\left(h_{1}\right), h_{2}\right\rangle \longrightarrow 0$.

Mostraremos que $A$ tem a propriedade DP1, isto é, $f\left(T_{n}^{*} T_{n}\right) \longrightarrow 0$, para todo $f \in A^{*}$.

Seja $f \in A^{*}$. Então, pelo Teorema de Hahn-Banach (1.1.3), existe $\tilde{f} \in K(H)^{*}$ tal que $\tilde{f} \uparrow_{A}=f$. Mas, pelo Teorema 4.1.30, temos que $K(H)^{*} \cong \mathscr{L}_{1}(H)$. Então, existe $S \in \mathscr{L}_{1}(H)$ tal que $\tilde{f}=\Phi_{S}$, isto é, $\tilde{f}(U)=\Phi_{S}(U)=\operatorname{tr}(U S)$, para todo $U \in K(H)$.

Seja $\mathscr{B}$ uma base ortonormal de $H$, temos que

$$
\begin{aligned}
\tilde{f}\left(T_{n}^{*} T_{n}\right) & =f\left(T_{n}^{*} T_{n}\right)=\Phi_{S}\left(T_{n}^{*} T_{n}\right)=\operatorname{tr}\left(T_{n}^{*} T_{n} S\right)=\sum_{\mathscr{B}}\left\langle T_{n}^{*} T_{n} S(e), e\right\rangle \\
& =\sup \left\{\sum_{\mathscr{B}^{\prime}}\left\langle T_{n}^{*} T_{n} S(e), e\right\rangle: \mathscr{B}^{\prime} \subset \mathscr{B}, \text { finito }\right\} .
\end{aligned}
$$

Como $\left\langle T_{n}^{*} T_{n}\left(h_{1}\right), h_{2}\right\rangle \longrightarrow 0$, para todo $h_{1}, h_{2} \in H$ e $S(e) \in H$, então $\left\langle T_{n}^{*} T_{n} S(e), e\right\rangle \longrightarrow 0$. Dado $\varepsilon>0$, existe $n_{0} \in \mathbb{N}$ tal que

$$
\begin{array}{cc}
\left|\left\langle T_{n}^{*} T_{n} S(e), e\right\rangle\right|<\frac{\varepsilon}{2\left|\mathscr{B}^{\prime}\right|} & \left(\forall n \geq n_{0}, \forall \mathscr{B}^{\prime} \subset \mathscr{B}, \text { finito }\right) \\
\left|\sum_{\mathscr{B}}\left\langle T_{n}^{*} T_{n} S(e), e\right\rangle\right| \leq \sum_{\mathscr{B}^{\prime}}\left|\left\langle T_{n}^{*} T_{n} S(e), e\right\rangle\right|<\frac{\varepsilon}{2} \quad\left(\forall n \geq n_{0}, \forall \mathscr{B}^{\prime} \subset \mathscr{B}, \text { finito }\right) \\
\left|f\left(T_{n}^{*} T_{n}\right)\right|=\left|\sum_{\mathscr{B}}\left\langle T_{n}^{*} T_{n} S(e), e\right\rangle\right|=\sup _{\substack{\mathscr{B}^{\prime} \subset \mathscr{B} \\
\text { finito }}} \sum_{\mathscr{B}^{\prime}}\left|\left\langle T_{n}^{*} T_{n} S(e), e\right\rangle\right| \leq \frac{\varepsilon}{2}<\varepsilon & \left(\forall n \geq n_{0}\right)
\end{array}
$$

Assim, $f\left(T_{n}^{*} T_{n}\right) \longrightarrow 0$, para todo $f \in A^{*}$, então $T_{n}^{*} T_{n} \stackrel{w}{\longrightarrow}$ 0. Logo, pelo Teorema 4.4.4, $A$ tem a propriedade DP1. 
(c) $\Rightarrow$ (d) Seja $\left(T_{n}\right)_{n} \subset A$ tal que $T_{n} \stackrel{w}{\longrightarrow} 0$. Como $A$ tem a propriedade DP, então, pelo Teorema 4.4.2, $T_{n}^{*} T_{n} \stackrel{w}{\longrightarrow} 0$ em $A$. Então, para todo $\varphi \in A^{*}, \varphi\left(T_{n}^{*} T_{n}\right) \longrightarrow 0$.

Analogamente à demonstração de $(\mathbf{a}) \Rightarrow(\mathbf{b})$, segue que $\left\|T_{n}(h)\right\| \longrightarrow 0$.

(d) $\Rightarrow$ (c) Seja $\left(T_{n}\right)_{n} \subset A$ tal que $T_{n} \stackrel{w}{\longrightarrow} 0$. Por hipótese, $\left\|T_{n}(h)\right\| \longrightarrow 0$, para todo $h \in H$. Analogamente à demonstração de $(\mathbf{b}) \Rightarrow(\mathbf{a})$, segue que $T_{n}^{*} T_{n} \stackrel{w}{\longrightarrow} 0$. Logo, pelo Teorema 4.4.2, $A$ tem a propriedade DP. 


\section{Referências Bibliográficas}

[1] M. D. Acosta, A. M. Peralta, The alternative Dunford-Pettis property for subspaces of the compact operators, Positivity 10 (2006), 51-63.

[2] C. A. Akemann, P. G. Dodds, J. L. B. Gamlen, Weak compactness in the dual space of a $C^{*}$-algebra, J. Funct. Anal. 10 (1972), 446-450.

[3] C. A. Akemann, G. K. Pedersen, Ideal perturbations of elements in $C^{*}$-algebras, Math. Scand. 41 (1977), 117-139.

[4] J. Arazy, Linear topological classification of $C^{*}$-algebras, Math. Scand. 52 (1983), 89-111.

[5] E. F. Beckenbach, R. Bellman, Inequalities, Springer Verlag Berlin, 1961.

[6] B. Blackadar, Operator Algebras: Theory of $C^{*}$-Algebras and von Neumann Algebras, Springer, 2006.

[7] G. Botelho, D. Pellegrino, E. Teixeira, Fundamentos de Análise Funcional, SBM, 2012.

[8] O. Bratteli, D. W. Robinson, Operator Algebras and Quantum Statistical Mechanics 1, Springer, 2002.

[9] L. J. Bunce, The Dunford-Pettis property in the predual of a von Neumann algebra, Proc. Amer. Math. Soc. 116 (1992), 99-100.

[10] L. J. Bunce, A. M. Peralta, The alternative Dunford-Pettis property in $C^{*}$-algebras and von Neumann preduals, Proc. Amer. Math. Soc. 131 (2002), 1251-1255.

[11] C. H. Chu, B. Iochum, The Dunford-Pettis property in $C^{*}$-algebras, Studia Math. 97 (1990), $59-64$.

[12] C. H. Chu, B. Iochum, S. Watanabe, $C^{*}$-algebras with the Dunford-Pettis property, Function Spaces (ed. K. Jarosz; Marcel Decker, New York 1992), 67-70.

[13] C. H. Chu, P. Mellon, The Dunford-Pettis property in JB*-triples, J. London Math. Soc. 55 (1997), 515-526.

[14] J. B. Conway, A Course in Functional Analysis, Springer-Verlag, 1985.

[15] W. J. Davis, T. Figiel, W. B. Johnson, A. Pelczynski, Factoring weakly compact operators, J. Funct. Anal. 17 (1974), 311-327.

[16] J. Diestel, A survey of results to the Dunford-Pettis property, in Proceedings of the Conference on Integration, Topology and Geometry in Linear Spaces, 2, Amer. Math. Soc., 15-60.

[17] N. J. Dunford, B. J. Pettis, Linear operations on summable functions, Trans. Amer. Math. Soc. 47 (1940), 323-392.

[18] R. Engelking, General Topology, Heldermann Verlag Berlin, 1989. 
[19] M. Fabian, P. Habala, P. Hájek, V. M. Santalucía, J. Pelant, V. Zizler, Functional Analysis and Infinite-Dimensional Geometry, Springer-Verlag, 2001.

[20] F. J. Fernández-Polo, A. M. Peralta, Compact tripotents and the Stone-Weierstrass theorem for $C^{*}$-algebras and $J B^{*}$-triples, J. Operator Theory 58 (2007), 157-173.

[21] W. Freedman, An alternative Dunford-Pettis property, Studia Math. 125 (1997), 143-159.

[22] A. Grothendieck, Sur les applications linéaires faiblement compactes d'espaces du type $C(K)$, Canad. J. Math. 5 (1953), 129-173.

[23] M. Hamana, On linear topological properties of some $C^{*}$-algebras, Tohoku Math. J. 29 (1977), $157-163$.

[24] A. J. Hissadomi, Propriedade de Dunford-Pettis Polinomial e Espaços Polinomialmente de Schur, Dissertação de Mestrado, Instituto de Matemática e Estatística, Universidade de São Paulo, Brasil, 1998.

[25] M. Martín, A. M. Peralta, The alternative Dunford-Pettis property in the predual of a von Neumann algebra, Studia Math. 147 (2001), 197-200.

[26] R. E. Megginson, An Introduction to Banach Space Theory, Springer-Verlag, 1998.

[27] S. M. Moshtaghuoun, J. Zafarani, Weak sequential convergence in the dual of operator ideals, J. Operator Theory 49 (2003), 143-151.

[28] G. J. Murphy, $C^{*}$-Algebras and Operator Theory, Academic-Press, London, 1990.

[29] G. K. Pedersen, $C^{*}$-Algebras and their Automorphism Groups, Academic Press, 1979.

[30] S. Sakai, $C^{*}$-Algebras and $W^{*}$-Algebras, Springer, 1970.

[31] C. Stegall, Duals of certain spaces with the Dunford-Pettis property, Notices Amer. Math. Soc. 19 (1972), 799

[32] M. Takesaki, Theory of Operator Algebras I, Springer-Verlag, 1979.

[33] M. Zippin, A remark on bases and reflexivity in Banach spaces, Israel J. Math. 6 (1968), 74-79. 